Portland State University

PDXScholar

$1-1-2011$

\title{
Development of a Hybrid Atomic Force and Scanning Magneto-Optic Kerr Effect Microscope for Investigation of Magnetic Domains
}

Andrew James Lawrence

Portland State University

Follow this and additional works at: https://pdxscholar.library.pdx.edu/open_access_etds Let us know how access to this document benefits you.

Recommended Citation

Lawrence, Andrew James, "Development of a Hybrid Atomic Force and Scanning Magneto-Optic Kerr Effect Microscope for Investigation of Magnetic Domains" (2011). Dissertations and Theses. Paper 147. https://doi.org/10.15760/etd.147

This Thesis is brought to you for free and open access. It has been accepted for inclusion in Dissertations and Theses by an authorized administrator of PDXScholar. Please contact us if we can make this document more accessible: pdxscholar@pdx.edu. 
Development of a Hybrid Atomic Force and Scanning Magneto-Optic

Kerr Effect Microscope for Investigation of Magnetic Domains

$$
\text { by }
$$

\title{
Andrew James Lawrence
}

A thesis submitted in partial fulfillment of the requirements for the degree of

\author{
Master of Science \\ in \\ Physics
}

Thesis Committee: Erik Sánchez, Chair John Freeouf Dean Atkinson

\section{Portland State University}

(C) 2011 


\begin{abstract}
We present the development of a far-field magneto-optical Kerr effect microscope. An inverted optical microscope was constructed to accommodate Kerr imaging and atomic force microscopy. In Kerr microscopy, magnetic structure is investigated by measuring the polarization rotation of light reflected from a sample in the presence of a magnetic field. Atomic force microscopy makes use of a probe which is scanned over a sample surface to map the topography. The design was created virtually in SolidWorks, a three-dimensional computer-aided drafting environment, to ensure compatibility and function of the various components, both commercial and custommachined, required for the operation of this instrument. The various aspects of the microscope are controlled by custom circuitry and a field programmable gate array data acquisition card at the direction of the control code written in National Instrument LabVIEW. The microscope has proven effective for both Kerr and atomic force microscopy. Kerr images are presented which reveal the bit structure of magnetooptical disks, as are atomic force micrographs of an AFM calibration grid. Also discussed is the future direction of this project, which entails improving the resolution of the instrument beyond the diffraction limit through near-field optical techniques. Preliminary work on fiber probe designs is presented along with probe fabrication work and the system modifications necessary to utilize such probes.
\end{abstract}




\section{Acknowledgments}

I wish to thank the members of the Sánchez Nano-Development Lab in the physics department of Portland State University. This work could not have been successful without their continued advice and support. These include undergraduate research students Cliff Kim, Joe Parks, Kyle Juedes, Allan Dunham, Justin Hiester, and Noah Brummer; Master's students Deepak Vedhachalam, Philip Witham, and John Mitchell; and doctoral students Dr. Mohan Vattipalli, Randy Smith, Richard Swinford, Jeff Doughty, and Mike DeArmond. Zechariah Dzegede contributed directly to this project with work on the LabVIEW code, and Amber Lauer designed and built the electromagnet. The author is particularly grateful for the assistance and guidance of Dr. Derek Nowak. His initial development of the microscope control systems was absolutely essential to the success of this project, and his additional contributions cannot be overstated.

I am thankful to Portland State University for the use of their facilities, and specifically the physics department for academic and financial support, as well as the PSU support staff, including Marc Nisenfeld and Chuck Heino for assistance with machining and Anne Keech and Kim Doty-Harris for administrative support.

I am also grateful to the various funding agencies who have supported this work. These include the National Science Foundation, the Office of Naval Research, the Western Institute of Nanoelectronics, the Semiconductor Research Corporation, the UC Discovery Grant, the Nanoelectronics Research Initiative, the Nanoelectronics Research Corporation, Intel, and the Oregon Nanoscience and Microtechnologies Institute.

I wish to thank my thesis committee for taking their time to evaluate this work. These include Dr. John Freeouf, Dr. Dean Atkinson, and advisor Dr. Erik J. Sánchez, to whom I am especially appreciative for his continued support and instruction.

Finally, and most importantly, I am grateful to my friends and family who have supported me all these years. 


\section{Table of Contents}

Abstract $\quad$ i

$\begin{array}{ll}\text { Acknowledgments } & \text { ii }\end{array}$

List of Figures $\quad$ v

List of Abbreviations viii

1 Introduction 1

2 Samples 5

2.1 Magneto-Optical Data Disks . . . . . . . . . . . . . . 5

2.2 Magnetic Glass . . . . . . . . . . . . . . . . . . . . . . 5

2.3 Thin Films . . . . . . . . . . . . . . . . . . . . 7

3 Optical Microscopy 9

3.1 History of Optical Microscopy . . . . . . . . . . . . . . . . . . . . . 9

3.2 Design of Optical Microscope . . . . . . . . . . . . . . . . . . 10

3.3 Construction of Optical Microscope . . . . . . . . . . . . . . . 14

3.4 Scanning Optical Microscopy . . . . . . . . . . . . . . . . . . . 15

3.5 Fluorescence Microscopy . . . . . . . . . . . . . . . . . . . . 17

3.6 Optical Resolution . . . . . . . . . . . . . . . . . 20

4 Atomic Force Microscopy 23

4.1 History of Scanning Probe Microscopy . . . . . . . . . . . . . . . 23

4.2 Basic Principles of AFM . . . . . . . . . . . . . . . . . . . . 24

4.3 Design of AFM . . . . . . . . . . . . . . . . . . . . . . . . . . . . . . . . .

4.4 AFM Electronics . . . . . . . . . . . . . . . . . . 31

4.5 Control of AFM . . . . . . . . . . . . . . . . . . . . . . . . . . . . . . . . . . . . . . . . . . 32

4.6 AFM Results . . . . . . . . . . . . . . . . . . 33 
5 Magneto-Optic Kerr Effect 35

5.1 History of Magneto Optics . . . . . . . . . . . . . . . . . 35

5.2 Mathematical Background on the Propagation of Light . . . . . . . 37

5.3 Light in a Medium . . . . . . . . . . . . . . . . . . 40

5.4 Mathematical Formulation of MOKE . . . . . . . . . . . 42

6 Magnetic Microscopy $\quad 46$

6.1 The Bitter Method . . . . . . . . . . . . . . . . . 46

6.2 Magnetic Force Microscopy . . . . . . . . . . . . . . . . . . 46

6.3 Electron Microscopy Techniques for Magnetic Imaging _ . . . . . . . 47

6.4 Kerr Microscopy . . . . . . . . . . . . . . . . . . . . 50

7 Writing Magnetic Domains $\quad 57$

7.1 Magnetic Domains . . . . . . . . . . . . . . . . 57

7.2 Design of Electromagnet . . . . . . . . . . . . . . . 58

7.3 Magnetic Field of a Circular Solenoid . . . . . . . . . . . . 60

7.4 Magnetic Field of a Square Solenoid . . . . . . . . . . . . . . . 62

7.5 Magnetolithography . . . . . . . . . . . . . . . . 65

8 Fiber Microscopy $\quad 68$

8.1 Optical and Atomic Force Microscopy with Fiber Probes . . . . . . 68

8.2 Probe Fabrication . . . . . . . . . . . . . . . . . . . . . . . . . 72

8.3 Fiber Microscopy Results . . . . . . . . . . . . . . . . . . . . . 73

8.4 Future Work . . . . . . . . . . . . . . . . . . . . . 73

$\begin{array}{lll}9 & \text { Conclusions } & 76\end{array}$

$\begin{array}{ll}\text { Bibliography } & 78\end{array}$

$\begin{array}{ll}\text { Appendix - CAD Drawings } & 86\end{array}$ 


\section{List of Figures}

1.1 Advancement in bit density of select data storage technologies since 1956. . . . . . . . . . . . . . . . . . . . 1

1.2 Bit density of near field MOKE compared with historical data storage technologies. . . . . . . . . . . . . . . . . . . 3

2.1 Tracks on a magneto-optical disk. The vertical arrows represent the magnetization of data bits. . . . . . . . . . . . . 6

3.1 Nikon Diaphot, modified for NSOM imaging. . . . . . . . . . . . . . . 12

3.2 SolidWorks model of microscope base plate. . . . . . . . . . . . . . 13

3.3 SolidWorks drawing of inverted optical microscope. a) Objective lens b) Sample holder c) Base plate d) Periscope mirrors e) Beam splitter f) CCD Camera . . . . . . . . . . . . . . . . . . . . . . 14

3.4 CCD image of PICI dye crystals, spin-coated on glass. . . . . . . 16

3.5 Reflection optical image of magnetic glass, Metglas 2605SA1, acquired at $1.0 \mathrm{~Hz}$ scan speed using $514 \mathrm{~nm}$ laser illumination at $100 \mu \mathrm{W}$. . . 18

3.6 Fluorescence spectrum of Rhodamine 6G. The solid blue line is absorption and the dashed red line is emission. . . . . . . . . . . . 19

3.7 Fluorescence image of Rhodamine 6G demonstrating photobleaching. 20

3.8 A cross section of magnetic glass reveals the resolution limit of our microscope to be less than $400 \mathrm{~nm}$. The $\mathrm{x}$ and y axes show lateral displacement in $\mu \mathrm{m}$ and relative light intensity in arbitrary units, respectively. The resolution is therefore given by $\Delta x \ldots \ldots \ldots 21$

3.9 Airy pattern produced by propagation through a circular aperture. . 22

4.1 SEM image of AFM tip. . . . . . . . . . . . . . . . . 25

4.2 Conceptual diagram of an AFM. . . . . . . . . . . . . 26

4.3 SolidWorks cross-section of scan head reveals piezo stack and tuning

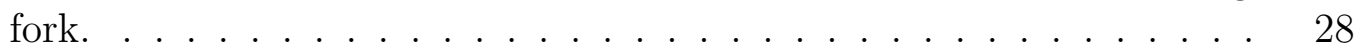

4.4 SolidWorks model of complete atomic force inverted optical microscope, including backplane assembly, scan head, and breakout box. . . 29

4.5 Photograph of functional atomic force microscope. . . . . . . . . 30

4.6 Phase image of AFM calibration grid. . . . . . . . . . . . 33

4.7 Topographic image of AFM calibration grid. . . . . . . . . . . 34 
5.1 Illustration of polar MOKE, in which the magnetization is normal to the surface. The disks represent the plane of polarization in the direction of the black arrows. . . . . . . . . . . . . . . . .

6.1 Simultaneous AFM topography (left) and MFM phase signals (right) reveal sectors of floppy data disk. . . . . . . . . . . . . .

6.2 Simultaneous AFM topography (left) and MFM phase (right) signals of MnGe film. . . . . . . . . . . . . . . . . . . . . . . . . . 48

6.3 Traditional configuration of Kerr microscope. . . . . . . . . . . . . 51

6.4 SolidWorks Diagram of Kerr Microscope a) Objective lens b) Scan stage c) Base plate d) Periscope assembly e) Beam splitter f) Polarizing beam splitter g) APD for s signal h) APD for $\mathrm{p}$ signal. . . . . . . . .

6.5 Updated GUI for scan program provides monitoring of three channels to display s, p, and difference signals simultaneously. The mirror pattern on the left of each image is an artifact of the closed loop scanner.

6.6 Kerr image of magneto-optic disk. a) s component of reflected light b) p component c) Difference signal revealing magnetic contrast along data tracks. . . . . . . . . . . . . . . . .

6.7 Three dimensional Kerr image reveals data bits on a magneto-optic disk. In this representation, the peaks corresponding to the bit structure are extremely apparent. . . . . . . . . . . . . . 56

7.1 SolidWorks rendering of vectorizable electromagnet. . . . . . . . . . . 59

7.2 Photograph of functional electromagnet. . . . . . . . . . . . . 59

7.3 GUI for electromagnet control software, written in LabVIEW. . . . . 60

7.4 Magnetic field of a circular loop. . . . . . . . . . . . . . . . 61

7.5 Magnetic field of a wire segment. . . . . . . . . . . . . . . . . . 62

7.6 Magnetic field of a square loop. . . . . . . . . . . . . . . 64

7.7 Preliminary attempt at writing a magnetic pattern in the shape of a

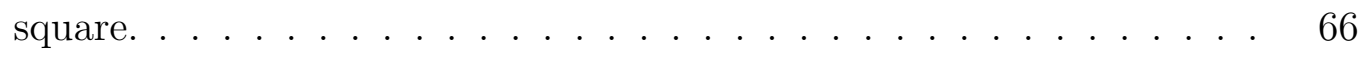

7.8 GUI for lithography program. . . . . . . . . . . . . . 67

8.1 Pulled optical fiber in various stages of FIB milling process. . . . . . 69

8.2 AFM sweep with long fiber attached to tuning fork. . . . . . . . . . . 70

8.3 AFM sweep with fiber glued to tuning fork circuit board. . . . . . . . 71

8.4 Illuminated fiber probe approaching sample surface. . . . . . . . . . . 71

8.5 CAD model of fiber probe for FDTD analysis. . . . . . . . . . . . . 72

8.6 Top down view of CAD fiber probe. . . . . . . . . . . . . . . . . 72

8.7 Gold coated fiber. FOV $=80 \mu \mathrm{m} \ldots \ldots \ldots$. . . . . . . . . . . . . . . . . . . . 73

8.8 First cut on fiber probe. . . . . . . . . . . . . . . . . . . 73

8.9 Fiber probe with second gold coating. . . . . . . . . . . . . 73

8.10 Bitmap used for bowtie cut. . . . . . . . . . . . . . . . . . . 74

8.11 Fiber after bowtie cut. . . . . . . . . . . . . . . . . . . . . . 74

8.12 Bowtie probe. . . . . . . . . . . . . . . 74 
8.13 Top down view of probe. . . . . . . . . . . . . . . . . 74

8.14 Simultaneous topographic (top) and optical transmission (bottom) images of gold film using a pulled optical fiber. . . . . . . . . . . . . . . 75 


\section{List of Abbreviations}

$\begin{array}{ll}\text { 3-D } & \text { Three-Dimensional } \\ \text { ANSOM } & \text { Apertureless Near-Field Scanning Optical Microscope (Microscopy) } \\ \text { AFM } & \text { Atomic Force Microscope (Microscopy) } \\ \text { APD } & \text { Avalanche PhotoDiode } \\ \text { CAD } & \text { Computer-Aided Design } \\ \text { CCD } & \text { Charge-Coupled Device } \\ \text { CD } & \text { Compact Disc } \\ \text { DAQ } & \text { Data AcQuisition (card) } \\ \text { DDS } & \text { Direct Digital Synthesis } \\ \text { DVD } & \text { Digital Versatile Disc } \\ \text { DXF } & \text { Drawing eXchange Format } \\ \text { EFM } & \text { Electrostatic Force Microscope (Microscopy) } \\ \text { FDTD } & \text { Finite-Difference Time-Domain } \\ \text { FPGA } & \text { Field-Programmable Gate Array } \\ \text { FIB } & \text { Focused Ion Beam Microscope } \\ \text { GUI } & \text { Graphical User Interface } \\ \text { HDD } & \text { Hard Disk Drive } \\ \text { MBE } & \text { Molecular Beam Epitaxy } \\ \text { MFM } & \text { Magnetic Force Microscope (Microscopy) } \\ \text { MO } & \text { Magneto-Optical } \\ \text { MOKE } & \text { Magneto-Optic Kerr Effect } \\ \text { NSOM } & \text { Near-Field Scanning Optical Microscope (Microscopy) } \\ \text { PCB } & \text { Printed Circuit Board } \\ \text { PBS } & \text { Polarizing Beam Splitter } \\ \text { PICI } & \text { Pseudoisocyanine Iodide } \\ \text { PID } & \text { Proportional-Integral-Differential } \\ \text { PMT } & \text { Photomultiplier Tube } \\ \text { RE-TM } & \text { Rare Earth - Transition Metal alloy } \\ \text { RF } & \text { Radio Frequency } \\ \text { SEM } & \text { Scanning Electron Microscope (Microscopy) } \\ \text { SEMPA } & \text { Scanning Electron Microscopy with Polarization Analysis } \\ \text { SICM } & \text { Scanning Ion Conductance Microscope (Microscopy) } \\ \text { SPM } & \text { Scanning Probe Microscope (Microscopy) } \\ \text { STM } & \text { Scanning Tunneling Microscope (Microscopy) } \\ \text { TEM } & \text { Transmission Electron Microscope (Microscopy) } \\ \text { TENOM } & \text { Tip-Enhanced Near Field Optical Microscope (Microscopy) } \\ & \end{array}$




\section{Introduction}

The first hard disk drive was invented by IBM in 1956. The drive weighed over a ton and stored 2000 bits/in ${ }^{2}$ at a price of roughly 100 bytes per dollar[1]. Since that time, technology has improved dramatically. In May of 2011, Seagate Technology broke the 1 terabyte barrier for a single hard drive platter[2], which translates to a bit density of 625 gigabits $/$ in $^{2}\left(3 \times 10^{8}\right.$ times the density of the first HDD $)$ at a price of $3 \times 10^{10}$ bytes per dollar (again $3 \times 10^{8}$ times better than in 1956).

\section{History of Data Storage}

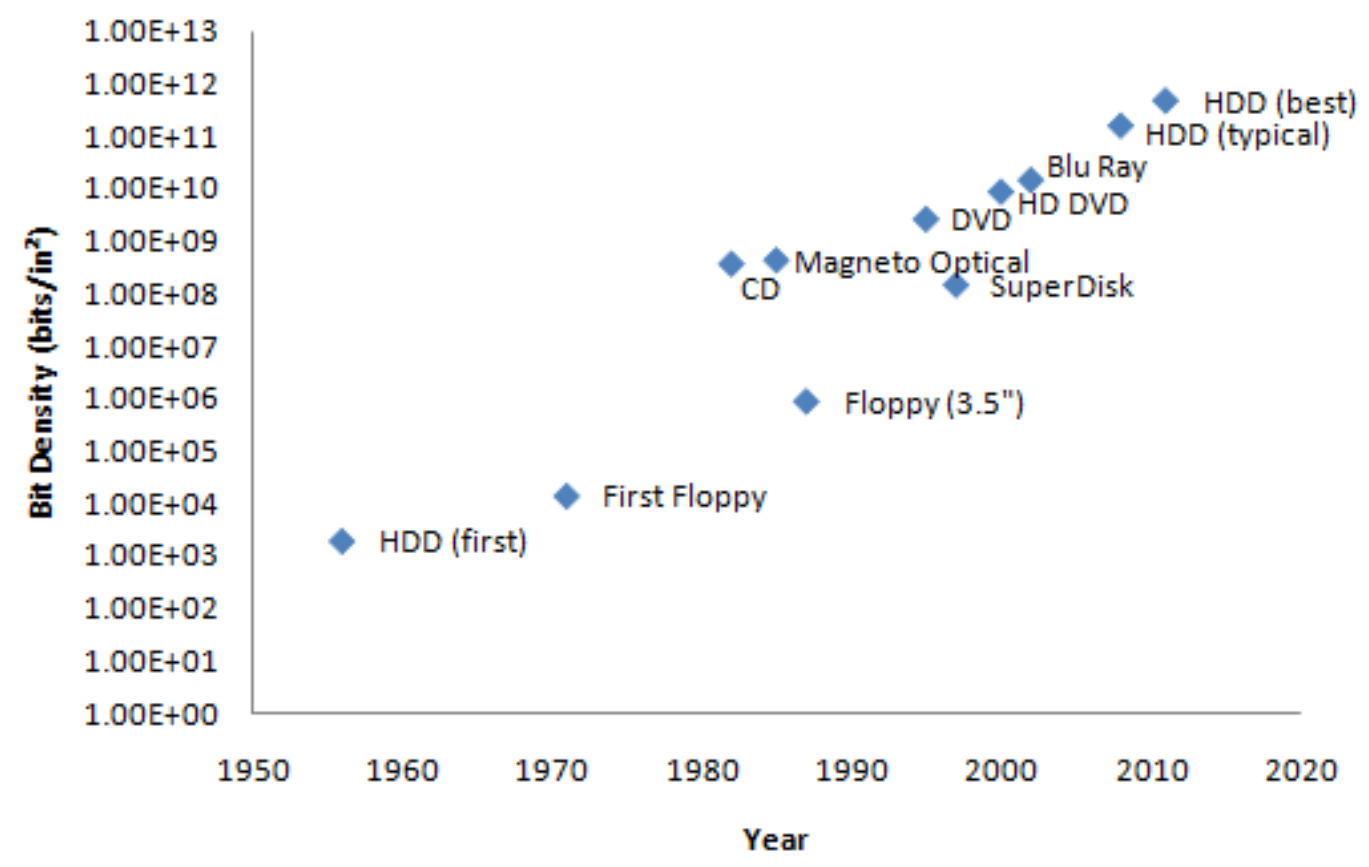

Figure 1.1: Advancement in bit density of select data storage technologies since 1956.

The rise in data storage density, as illustrated in figure 1.1, has been instrumental in bringing computers from the room-filling behemoths of the 1950s to the personal computers we know today. As technology has progressed, we have seen steep rises (note the logarithmic scale of the y-axis) in the bit density of both optical (compact 
discs through Blu-ray discs) and non-optical (floppy and hard disk drives) media. And while optical media has traditionally held a slight edge over non-optical in this respect, optical technologies are fundamentally limited by the diffraction of light.

Blu-ray technology, which represents the state of the art in optical storage, uses blue light with a wavelength of $405 \mathrm{~nm}$, and is therefore limited in resolution to about $175 \mathrm{~nm}$. This translates to a bit density of $1.15 \times 10^{11} \mathrm{bits} / \mathrm{in}^{2}$ in a perfect theoretical system; the constraints of the real world bring the actual number down by nearly an order of magnitude to $1.34 \times 10^{10} \mathrm{bits} / \mathrm{in}^{2}$. Even the theoretical ideal, however, is roughly equivalent to that of a typical consumer hard drive and well below the state of the art for non-optical media. No amount of optimization can bring the data density of optical storage technologies to the level currently available in hard disk drives.

One might then be tempted to assume that optical data storage will soon prove obsolete or be relegated to niche markets. This is not necessarily the case. The diffraction limit applies only in the far field-that is, more than one wavelength from the source[3]. The evanescent field within one wavelength of the source (the so-called near-field) is non-propagating and thus not subject to diffraction. It is this fact that is exploited in near field scanning optical microscopy (NSOM), in which an atomic force microscope (AFM) scans the sample surface with a fiber probe that detects this evanescent field. NSOM currently represents the most promising technique for increasing optical bit density beyond the constraints of the diffraction limit[4].

The two prevalent forms of optical data storage are compact discs (including DVDs and Blu-rays) and magneto-optical disks. CDs are obviously the more familiar of the two technologies, but are impractical for data storage at the nano-scale due to their reliance on propagating light and physical pits which would be extremely difficult to produce on such a scale. Magneto-optical (MO) disks, however, are well suited for nano-scale data storage. MO drives operate by reading the orientation of 
magnetic domains via the magneto-optic Kerr effect (MOKE) and rewriting these domains by laser heating the sample in the presence of a magnetic field. The bit size in such a device, therefore, is limited only by the resolution of the instrument. It is for this reason that we have endeavored to build an NSOM system with Kerr imaging capabilities. In such a system, the resolution is defined by the dimensions of the imaging probe, providing us with a theoretical resolution limit of $20 \mathrm{~nm}$. This translates to a bit density of $\sim 1.6 \times 10^{12} \mathrm{bits} / \mathrm{in}^{2}$, roughly five times greater than the most advanced storage technologies in existence today. Figure 1.2 places the predicted bit density of near field MOKE (assuming $20 \mathrm{~nm}$ lateral resolution) in its proper place among the aformentioned storage technologies.

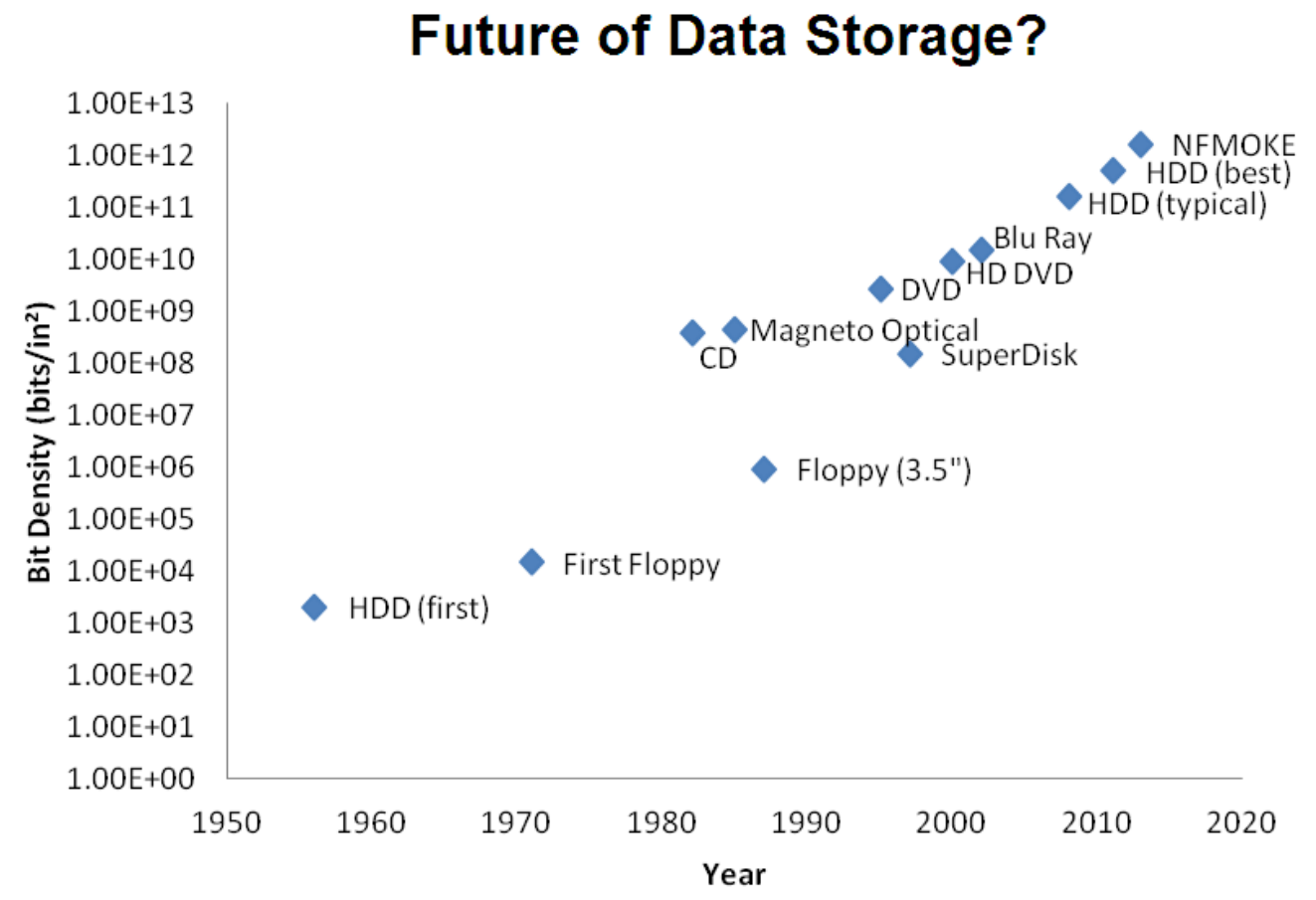

Figure 1.2: Bit density of near field MOKE compared with historical data storage technologies.

Presented here is the design, development, and construction of an inverted optical 
microscope with AFM and MOKE capabilities to serve as the base for the complete system. In chapter 2, we give background information on some samples of interest. Chapter 3 details the development and progression of the optical microscope, and the development of the atomic force microscope system is described in chapter 4 . The theoretical background on the mathematical formulation of the Kerr effect are provided in chapter 5. Chapter 6 discusses various forms of magnetic microscopy, specifically magnetic force microscopy (MFM) and Kerr microscopy, which are both of interest to this project. Our progress towards writing magnetic information is detailed in chapter 7 , and the conversion of the system to image with fiber probes is presented in chapter 8. Finally, Appendix A provides the mechanical drawings for all custom-designed components of the optical and atomic force microscope systems. 


\section{Samples}

The emphasis of this project is tool development rather than any specific application of the tool itself, such as material characterization or data storage. Therefore, no attempt was made to fabricate novel samples. Investigation was instead focused on well characterized samples such as magnetic thin films, magnetic glass, and magnetooptical data disks.

\subsection{Magneto-Optical Data Disks}

Magneto-optical (MO) data disks have proven to be an ideal sample for investigation by MOKE microscopy. This is not surprising when one considers that MO disk drives work on the same principles as our microscope. Indeed, MO disks are specifically tailored to be the optimal sample for a project such as this, as they have maximum Kerr rotation as well as a convenient Curie temperature by design.

These disks consist of a film ( $\sim 30 \mathrm{~nm})$ of rare earth-transition metal (RE-TM) alloys of the form $\left.\mathrm{Tb}_{y} \mathrm{Gd}_{1-y}\right)_{x}\left(\mathrm{Fe}_{z} \mathrm{Co}_{1-z}\right)_{1-x}$ between two layers of dielectric $\left(\mathrm{SiN}_{x}\right.$ or $\mathrm{AlN}_{x}, \sim 100 \mathrm{~nm}$ ) on a polycarbonate or glass substrate. The alloy layer is deposited by radio frequency $(\mathrm{RF})$ sputter coating, usually with an alloy target, with typical values for $\mathrm{x}, \mathrm{y}$, and $\mathrm{z}$ being approximately $0.2,0.9$, and 0.9 , respectively. The introduction of "pregrooves" on the substrate by etching, molding, or stamping creates topographic tracks on the surface of the film, $\sim 1-2 \mu \mathrm{m}$ wide, as illustrated in figure 2.1. The disk is then coated with a reflective, heat-sinking layer of metal $(\sim 50 \mathrm{~nm})$ and a final coating of protective lacquer $(\sim 10 \mu \mathrm{m})[5]$.

\subsection{Magnetic Glass}

So called magnetic glass is not glass in the conventional sense. Rather, it is a thin foil of amorphous magnetic alloy. The base is a ferromagnetic metal (typically iron, cobalt, or nickel), along with less than $10 \%$ of other metals such as boron, silicon, 


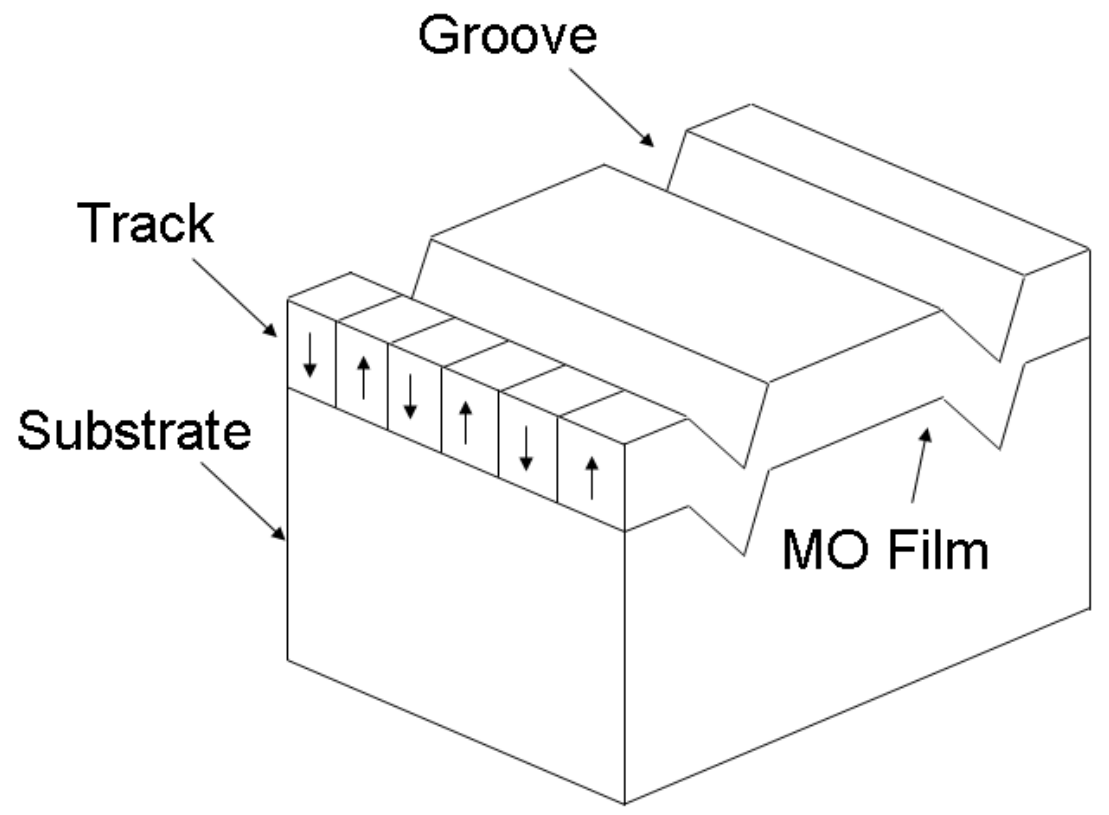

Figure 2.1: Tracks on a magneto-optical disk. The vertical arrows represent the magnetization of data bits.

chromium, and molybdenum. They are produced by rapid solidification at a rate of roughly $1 \times 10^{6} \mathrm{~K} / \mathrm{s}[6]$ - too fast for the atoms to reorient into an ordered crystal lattice.

The end result of this process is a sample with unique magnetic properties. Among these are high permeability, low core loss, and variable hysteresis curves. The cobaltbased alloy Metglas 2714A, for example, has a relative permeability of 1E6[7]; for comparison, nickel has a permeability of only about 100[8]. This translates to a magnetization in the alloy about $1 \times 10^{4}$ times higher than in nickel under the same conditions. Core loss is a measure of the power lost to heat or noise in a transformer or inductor. As magnetic glass is employed extensively in such applications, low core loss is a very desirable quality. The iron-based magnetic alloy Metglas 2605SA1 has a core loss of .29 W/kg at $60 \mathrm{~Hz}, 1.35 \mathrm{~T}[9]$. This corresponds to $40 \%$ of the core loss one would expect from grade M3 electrical steel. 
The hysteresis curves of magnetic glass can be tailored to a specific application by an anneal process. Metglas 2605SA1, when annealed in the absence of an external magnetic field, has a hysteresis curve which is nearly linear, whereas annealing in the presence of a field can result in a curve with a high squareness ratio. The cobalt-based Metglas 2705M, exhibits a remarkably high squareness ratio without annealing[10]. The unique properties of magnetic glass are ideally suited for many applications, including power distribution, power and current transformers, electromagnetic shielding, and magnetic sensors.

Our investigation was limited to Metglas Alloy 2605SA1 due to availability. As mentioned before, this is an iron-based alloy. By weight, it contains 85-95\% iron, $5-10 \%$ silicon, and 1-5\% boron, making it similar in composition to glasses previously imaged by similar techniques[11]. It is characterized by extremely low core loss and high permeability, and is therefore particularly well suited for application in power distribution. Also of note is the relatively low Curie temperature of $399^{\circ} \mathrm{C}$.

\subsection{Thin Films}

There are various magnetic thin films that may prove to be of interest for investigation with this instrument. Initial investigation has focused on simple elemental films of the ferromagnetic metals iron, nickel, and cobalt, all of which are readily available. These films were sputter coated (Plasma Sciences Inc. CrC-100 Sputtering System) on glass substrates. Of slightly more interest would be permalloy[12], an alloy containing about $20 \%$ iron and $80 \%$ nickel, which is notable for its extremely high permeability.

A promising sample is a multi-layer film consisting of 2-12 nm of nickel sandwiched between $2 \mathrm{~nm}$ cap layers of copper. This sample has been investigated by other groups[13], and the magnetic domain structure is well characterized. Initial attempts to fabricate a similar sample proved unsuccessful as our sputter coater lacked the precision inherent to molecular beam epitaxy (MBE), which is apparently critical to 
domain formation. Another sample of interest is a multi-layer film with alternating layers of $4 \AA$ cobalt and $10 \AA$ platinum[4]. This was attempted using a multi-target sputtering system (Lesker KJLC AXXIS). 


\section{Optical Microscopy}

Optical microscopy is a broad field, encompassing any instrument in which light is used to resolve features too small to be seen by the human eye. The most basic such instrument is a simple convex lens. The light reflected from an object is diverged by the lens, resulting in a magnified image. Magnifying glasses use this simple principle to make small objects visible, and thus represent the simplest form of optical microscopy; by contrast, optical microscopes can be much more sophisticated instruments, such as near-field scanning optical microscopes (NSOM) which image at higher resolution than is possible with a traditional microscope by illuminating the sample through an optical fiber.

\subsection{History of Optical Microscopy}

The first true microscope is believed to have been invented in the Netherlands by Hans and Zacharias Janssen in 1590, when the early opticians mounted two convex lenses in a telescoping tube, though some evidence attributes the invention to Galileo Galilei in 1609. Regardless of who first invented it, it was Galileo's instrument that

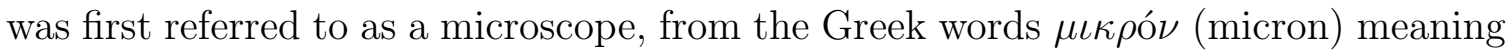
"small", and $\sigma \kappa o \pi \epsilon \tilde{\iota} \nu$ (skopein) meaning "to look at", when Giovanni Faber coined the word in 1624[14].

In the centuries that followed, scientists continued to improve upon the original design, gradually improving the capabilities of optical microscopy. Christiaan Huygens invented the first achromatically corrected eyepiece, known as the "Huygens ocular", in the late 17th century[15]. Robert Hooke became the first to construct a microscope with a built-in illumination source in $1655[16]$. His device closely resembled the familiar optical microscopes of today. In 1733, Chester Moore Hall invented the achromatic doublet[17], a refracting lens capable of correcting for chromatic aber- 
rations, and in 1830, Joseph Jackson Lister constructed a lens which minimized both chromatic and spherical aberration[18]. In 1893, August Köhler developed "Köhler illumination", a scheme for uniform illumination of the sample[19]. Frederik Zernike earned a Nobel Prize in 1953 for the invention of phase contrast microscopy, which allowed for the first imaging of live cells[20], and he was followed by Nomarski's invention of differential interference contrast in 1955[21].

Perhaps the most significant obstacle to optical microscopy has been the inherent resolution limit due to the diffraction of light. In 1874, Abbe described the diffraction limit, arguing that even with perfect optics, the resolution of an optical microscope was ultimately limited to roughly half of the wavelength of the illumination light. Richard Zsigmondy improved resolution in 1903 with his "ultramicroscope" by imaging scattered light instead of reflection[22] — work for which he won a Nobel Prize in 1925. Three years later, in 1928, E.H. Synge proposed a technique to beat the diffraction limit with point scanning through a small aperture[23]. Insufficient technology made this impossible to realize experimentally until 1972, when Ash and Nicholls imaged a diffraction grating using radiation of wavelength $3 \mathrm{~cm}[24]$. With the invention of the scanning tunneling microscope (STM) by Binnig and Rohrer in 1981[25], Synge's original vision of a point scanning optical microscope became possible by using a tapered optical fiber as the probe. This was first accomplished in 1984, simultaneously by Lewis[26], et al., and Pohl[27], et al., resulting in a resolution of 25 $\mathrm{nm}$, or one twentieth the wavelength of the illumination light. This technique became known as near-field scanning optical microscopy (NSOM).

\subsection{Design of Optical Microscope}

This design was originally conceived as a replacement for a heavily modified Nikon Diaphot Inverted Tissue Culture Microscope being converted into a tip-enhanced near field optical microscope (TENOM) system[28] [29], shown in figure 3.1. As the project 
continued to progress and further modification became necessary, it grew increasingly apparent that adaptation of an existing system would not afford us the flexibility we needed. We therefore endeavored to design a system from the ground up with an emphasis on stability, simplicity, and versatility.

We soon realized that, despite the intended simplicity, it would be nearly impossible to mentally visualize each of the various components of such a complex instrument and have any confidence in their placement, compatibility, or alignment. As an alternative, it was decided that the microscope should be constructed in a virtual environment using the 3-D CAD (computer-aided design) program SolidWorks. Each individual component was drafted and then brought together into one comprehensive assembly, providing a fast and free design phase in which all of the parts were guaranteed to fit together exactly as they were intended to.

Thorlabs, a well-known optical hardware vendor, proved instrumental in the design phase, as they provided nearly all of the optical and structural components of the microscope. The physical design of the optical microscope provides the optical pathways necessary for excitation and collection in this system, emulating the function of the Diaphot at a fraction of the price.

As discussed, an optical microscope can range in complexity from a tool as simple as a magnifying glass to an instrument as intricate as an NSOM system. In all cases, however, the basic concept remains the same. The sample is illuminated, resulting in an optical response, and this response is then collected to gain information about the sample. Therefore, the critical components of any optical microscope are the illumination source, the condenser lens which focuses the illumination light, the objective lens which magnifies the image, and the detector. Our design is for an inverted optical microscope, meaning the objective lens is located beneath the sample. Furthermore, we use an epi-illumination source, allowing the objective lens to double 


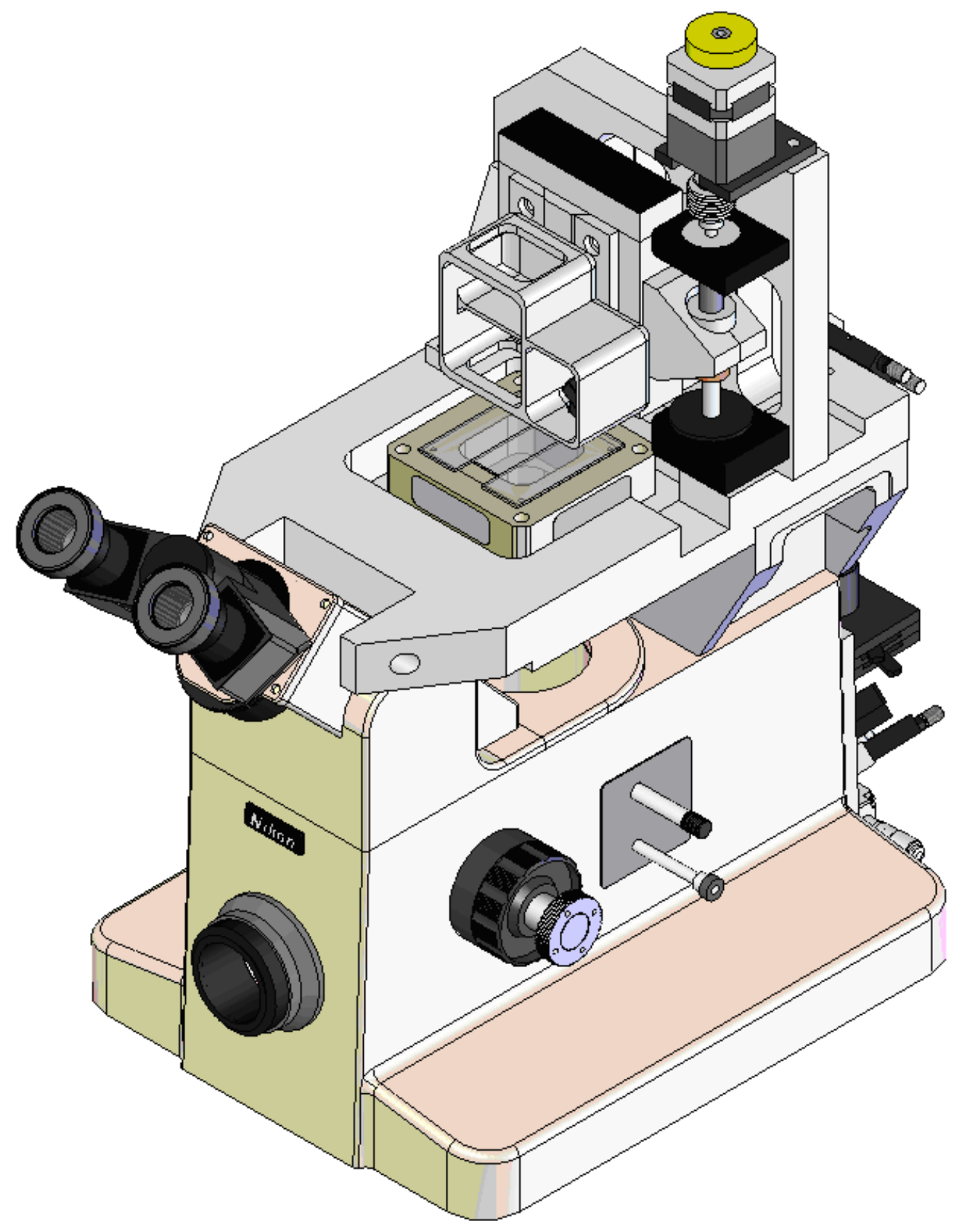

Figure 3.1: Nikon Diaphot, modified for NSOM imaging. 
as the condenser.

We began by constructing a stable base. Four 1.5" diameter posts (Thorlabs P8) are mounted to an optical breadboard (Thorlabs MB1824) and support the custom designed base plate. The base plate, seen in figure 3.2, supports the scan bed, a two axis nanopositioning stage (Physik Instrumente 733.2CL) on which the sample is mounted. The illumination source, an external laser, is brought into a cube-mounted beam splitter (Thorlabs CM1-BS013) by a periscope consisting of two kinematic mirror mounts (Thorlabs KM100) attached to single axis translation stages (Melles Griot 148-103), mounted to the optical breadboard and the bottom of the base plate.

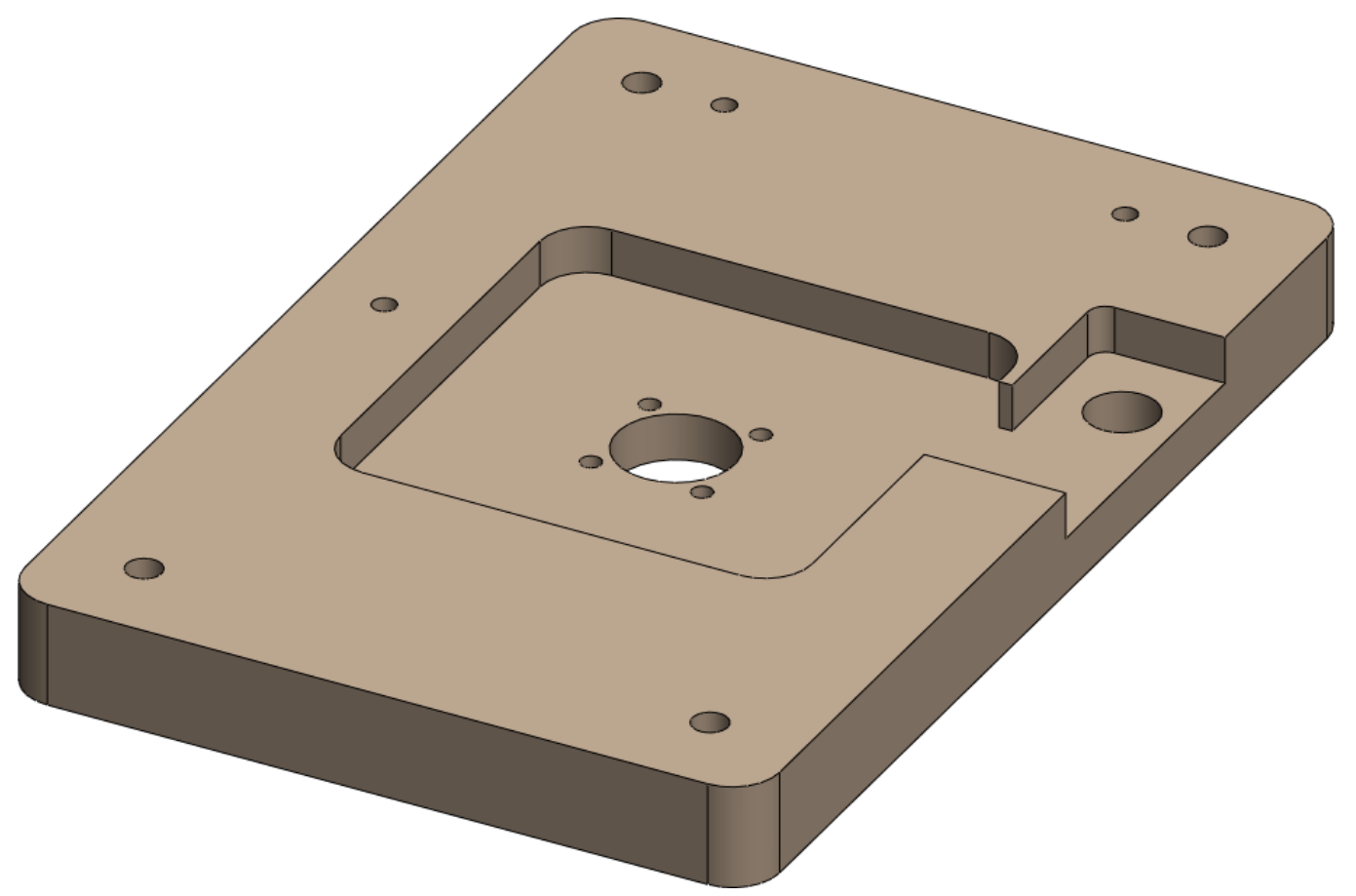

Figure 3.2: SolidWorks model of microscope base plate.

The beam splitter reflects the light to the objective lens (Zeiss 1036-022, 1.3NA, 100x Epiplan Neofluar), which is mounted in a z translation stage (Thorlabs SM1Z). This translator controls the distance between the objective and the sample, providing 
focusing capabilities. The light is reflected back down through the objective and beam splitter to a $90^{\circ}$ kinematic mirror mount (Thorlabs KCB1), which redirects the light to the detector.

The final design, shown in figure 3.3 is a highly adaptable, low-cost instrument with potential capabilities beyond those of any commercially available system. The design proved so effective and versatile that, apart from the MOKE system and the original TENOM system, it has also been adapted to suit two other projects in the lab - a confocal multi-laser excitation microscope and a scanning ion conductance atomic force microscope (SICM)[30].

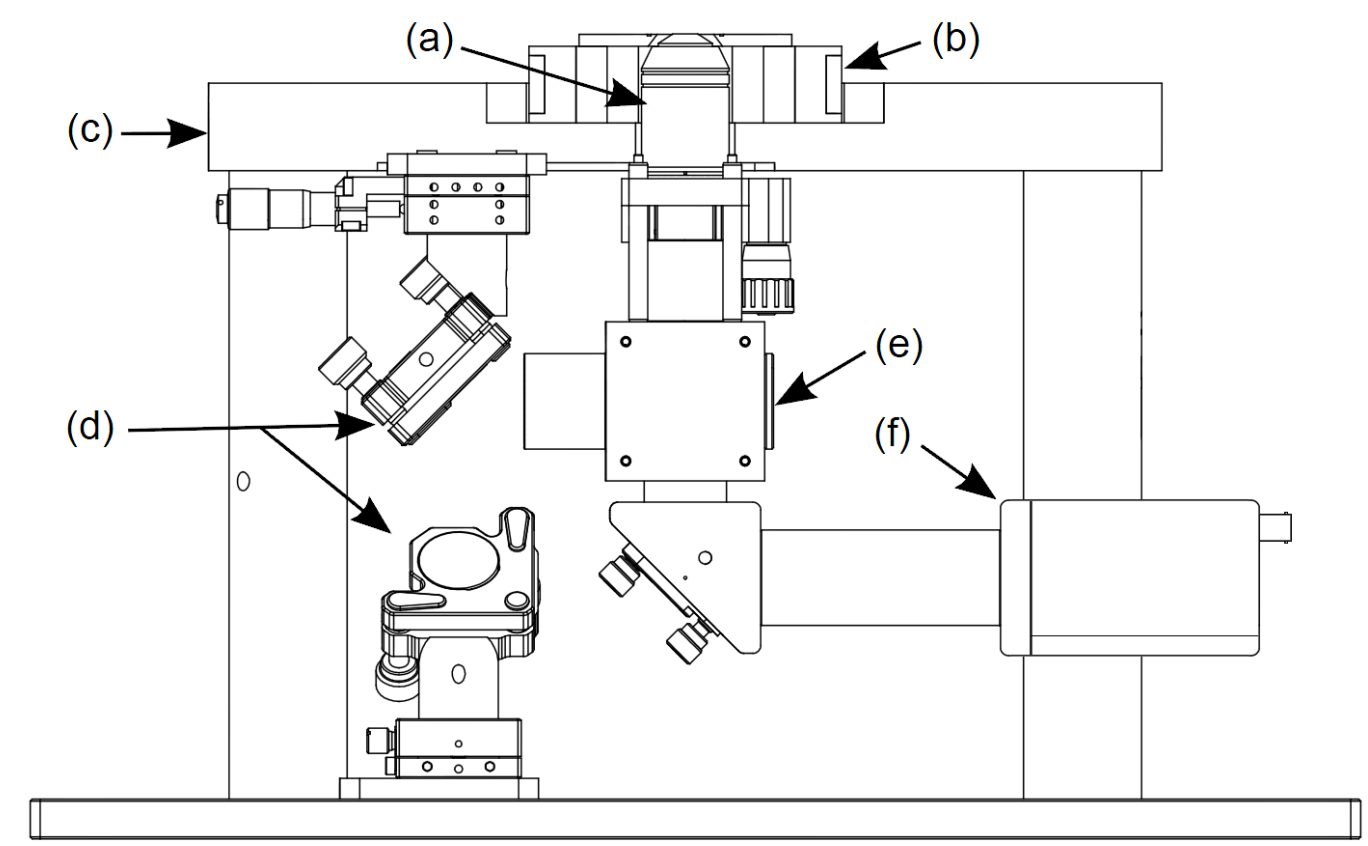

Figure 3.3: SolidWorks drawing of inverted optical microscope. a) Objective lens b) Sample holder c) Base plate d) Periscope mirrors e) Beam splitter f) CCD Camera

\subsection{Construction of Optical Microscope}

With a comprehensive design in hand, the physical construction of the microscope was fairly straightforward. As mentioned before, most of the components of the microscope were provided by Thorlabs. The various parts fit together using Thorlabs 
30mm cage system as well as their proprietary SM1 (1.035"-40) threading.

At this phase in the project, the only custom parts were the base plate and the aluminum mounting plates for the periscope mirrors. The periscope plates were easily machined based on the SolidWorks drawings, but the base plate presented a more challenging problem, being a large piece with many mounting holes, pockets, and external fillets. The mill (Bridgeport BR-54015) to which we had access was an antiquated machine with no digital readouts and so much play in the $\mathrm{x}$ and $\mathrm{y}$ axes that it proved entirely inadequate for any high-precision machining. Instead, the SolidWorks part was converted to a DXF (drawing exchange format) file and imported directly into a CNC (computer numerical control) mill. The technical drawings can be seen in Appendix A.

Upon assembly of the microscope, optical images were obtained almost immediately. A lens was placed in the detector section, carefully positioned in the lens tube using retaining rings (SM1RR), and a CCD (charge-coupled device) camera was attached using a C-Mount adapter (SM1A10). Figure 3.4 shows a CCD image of pseudoisocyanine iodide (PICI) dye crystals, spin-coated on glass. This image was obtained without the periscope assembly, instead making use of a fiber light for transillumination.

\subsection{Scanning Optical Microscopy}

The instrument of course functions well as a simple optical microscope, but the addition of point-scanning capabilities requires integration of scanning and collection mechanisms. The necessary algorithms are written in LabVIEW and controlled

through a National Instruments data acquisition (DAQ) card (PCIe-7852R) with a field programmable gate array (FPGA) processor (discussed further in section 4.4).

The scan algorithm generates two synchronized triangle waveforms, one for the $\mathrm{x}$-axis at the scan frequency, and one for the $\mathrm{y}$-axis with a longer period depending on 


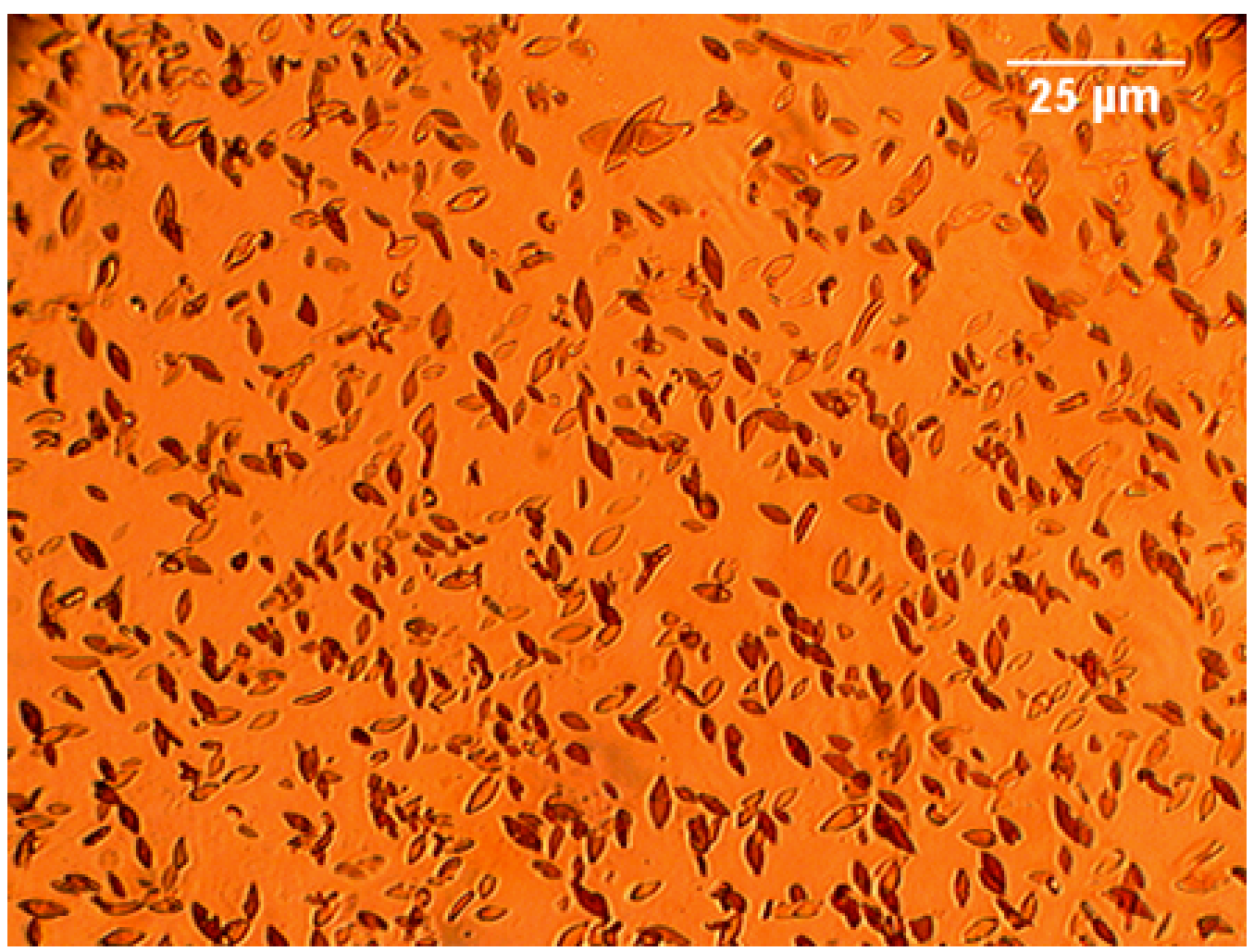

Figure 3.4: CCD image of PICI dye crystals, spin-coated on glass.

the resolution of the scan. For example, a $1 \mathrm{~Hz}$ scan at 10x10 pixel resolution would be composed of a $1 \mathrm{~Hz}$ triangle wave in the $\mathrm{x}$-axis and a $0.1 \mathrm{~Hz}$ triangle wave in the y-axis, resulting in a raster scan pattern. The scan size is controlled by adjusting the amplitudes of these signals using a programmable voltage divider (Analog Devices AD5290)[31]. These signals are then summed (OP471 operational amplifier) with a static voltage offset to adjust the origin of the region of interest[31]. The output waveform is applied to the controller of a piezo-driven nanopositioning stage (Physik Instrumente P-733.2CL) to which the sample is mounted, causing the sample to be scanned in relation to the objective lens. The stage provides closed loop travel in two axes over $100 \mu \mathrm{m}$ with $<2 \mathrm{~nm}$ repeatability and $0.3 \mathrm{~nm}$ resolution[32].

The CCD camera was replaced with an avalanche photodetector, or APD (Thor- 
labs APD110A). The implementation of point-scanning capabilities allows imaging of a much larger area at increased resolution by measuring the light intensity at each pixel. The field of view in such a setup is limited by the range of the nanopositioning stage to $(100 \mu \mathrm{m})^{2}$, while the resolution is limited by diffraction to roughly half the wavelength of the illumination light $(\lambda / 2)$. Our primary illumination sources were an $\operatorname{argon}$ laser $(\lambda=514 \mathrm{~nm})$ and an infrared diode laser $(\lambda=833 \mathrm{~nm})$. Figure 3.5 shows a point scan of magnetic glass (Metglas 2605SA1) acquired at a $1.0 \mathrm{~Hz}$ scan speed using $514 \mathrm{~nm}$ laser illumination at $100 \mu \mathrm{W}$.

\subsection{Fluorescence Microscopy}

Fluorescence is the process by which light is emitted from a substance after absorption of light of a different wavelength. This occurs due to the relaxation of an orbital electron after excitation by the incident light. The excitation can be described by

$$
S_{0}+h \nu_{i} \rightarrow S_{1}
$$

and the fluorescence by

$$
S_{1} \rightarrow S_{0}+h \nu_{s}+\text { heat }
$$

where $S_{0}$ represents the ground state, $S_{1}$ the first excited state, and $h \nu_{i}$ and $h \nu_{s}$ are the energies of the incident and scattered light, respectively[33].

So, whereas traditional optical microscopes function by collecting the light reflected from the sample, fluorescence microscopes must collect only the emitted light by filtering out the wavelength of the reflected light. With the fluorophore returning to the ground state, it would seem that fluorescence could continue indefinitely; in reality, the fluorophore eventually loses its ability to fluoresce, a phenomenon known as photobleaching. The exact mechanism by which this occurs is undetermined. In short, fluorescence microscopy detects changes in wavelength using notch filters. This 


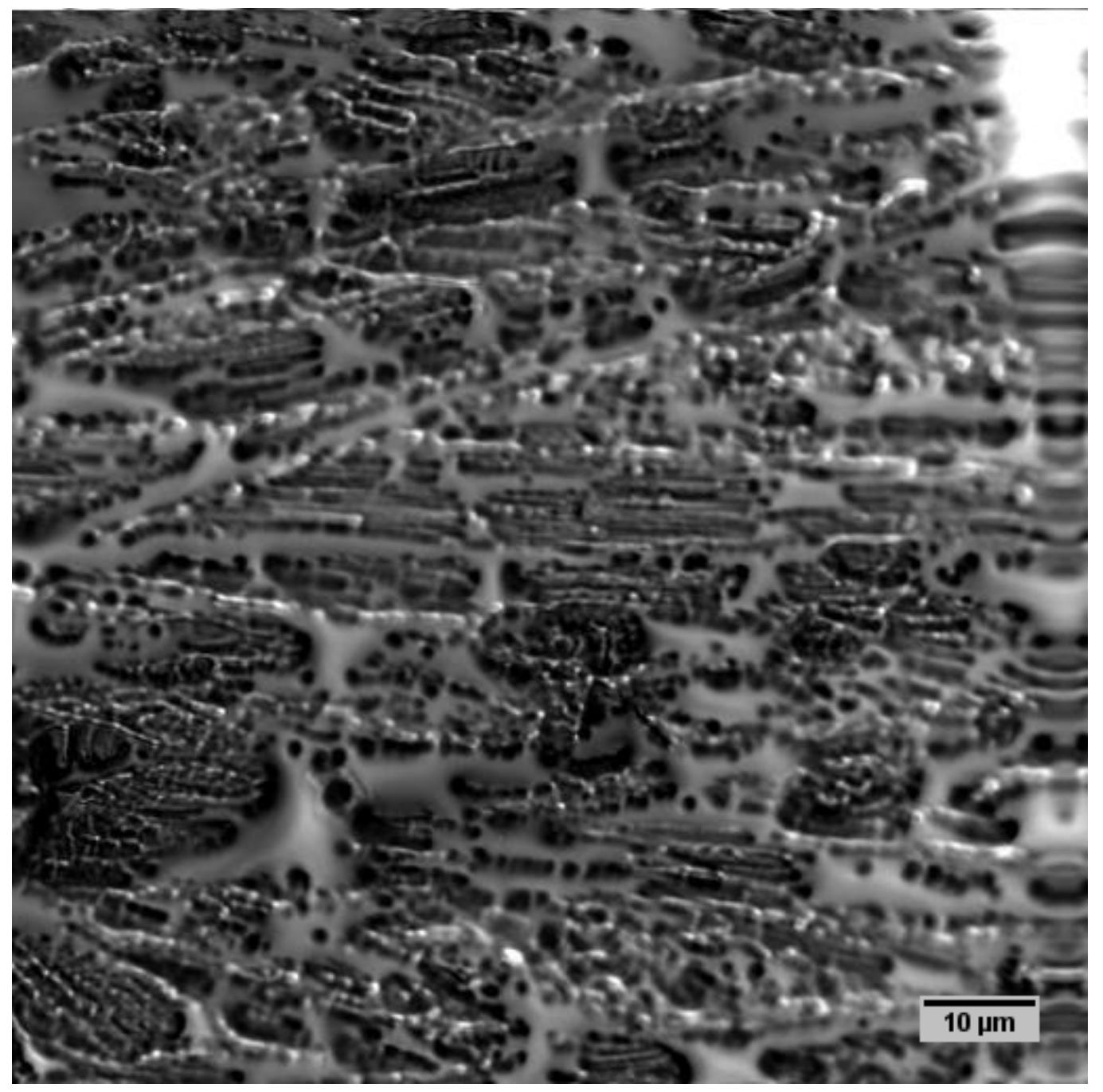

Figure 3.5: Reflection optical image of magnetic glass, Metglas 2605SA1, acquired at $1.0 \mathrm{~Hz}$ scan speed using $514 \mathrm{~nm}$ laser illumination at $100 \mu \mathrm{W}$. 
is analogous to Kerr microscopy, in which the goal is to detect changes in polarization using polarizing filters. Therefore, to verify proper operation of the microscope, it was useful to set it up in fluorescence mode before attempting Kerr imaging.

We chose a sample of Rhodamine $6 \mathrm{G}\left(\mathrm{C}_{28} \mathrm{H}_{31} \mathrm{~N}_{2} \mathrm{O}_{3} \mathrm{Cl}\right)$, a fluorescent dye often used as a gain medium in dye lasers. R6G has its absorption peak at $528 \mathrm{~nm}[34]$, so we selected the strongest line of an argon laser $(514 \mathrm{~nm})$ as the illumination source. Another notch filter was placed before the photon detector to collect only $633 \mathrm{~nm}$ light. The emission and absorption spectra of R6G[34] are shown in figure 3.6.

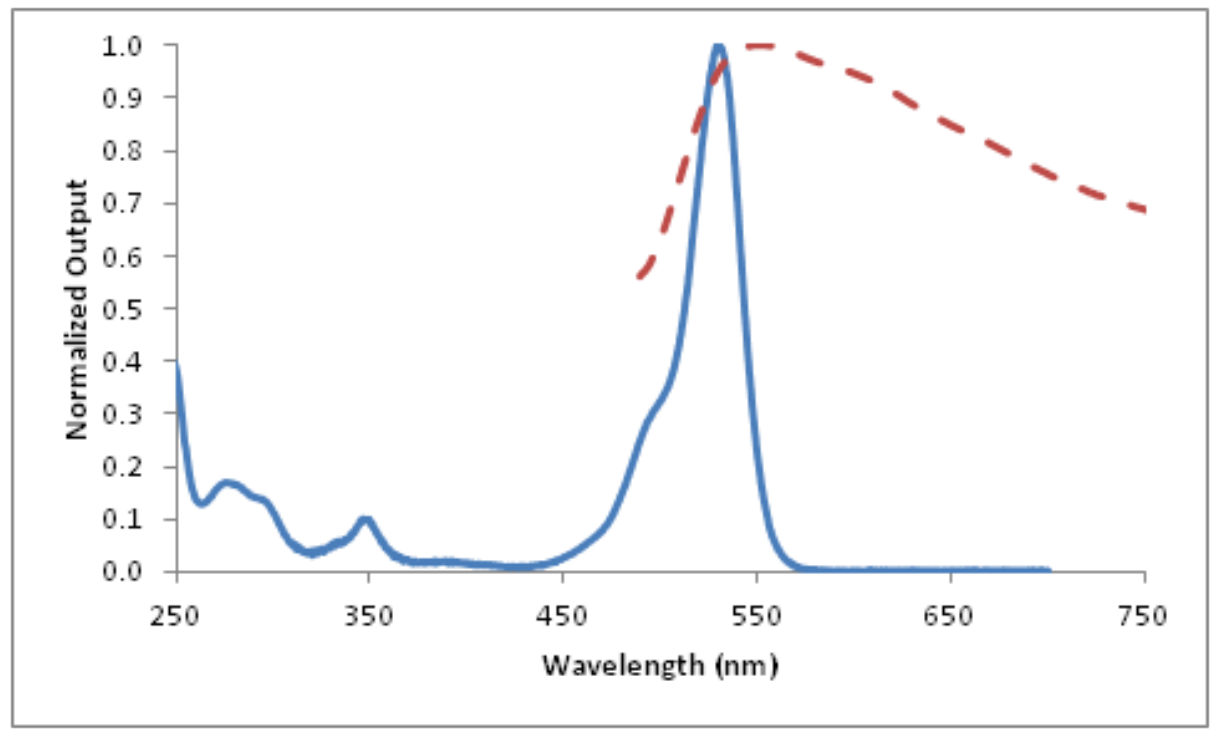

Figure 3.6: Fluorescence spectrum of Rhodamine 6G. The solid blue line is absorption and the dashed red line is emission.

With the filters in place, any light being detected was necessarily a result of the dye fluorescing. However, optical systems are always imperfect, so to verify that we were indeed imaging in fluorescence mode as opposed to simply collecting a reflection, we employed the scan algorithm as a makeshift nanolithography tool, effectively writing concentric squares by scanning sequentially larger areas. The first, innermost square then became the most photobleached as it was subjected to the illumination light on 
the first scan and each successive scan. The second scan area then became the second most photobleached and so on. This technique of successive photobleaching proved quite effective. In figure 3.7, at least four distinct regions are clearly seen.

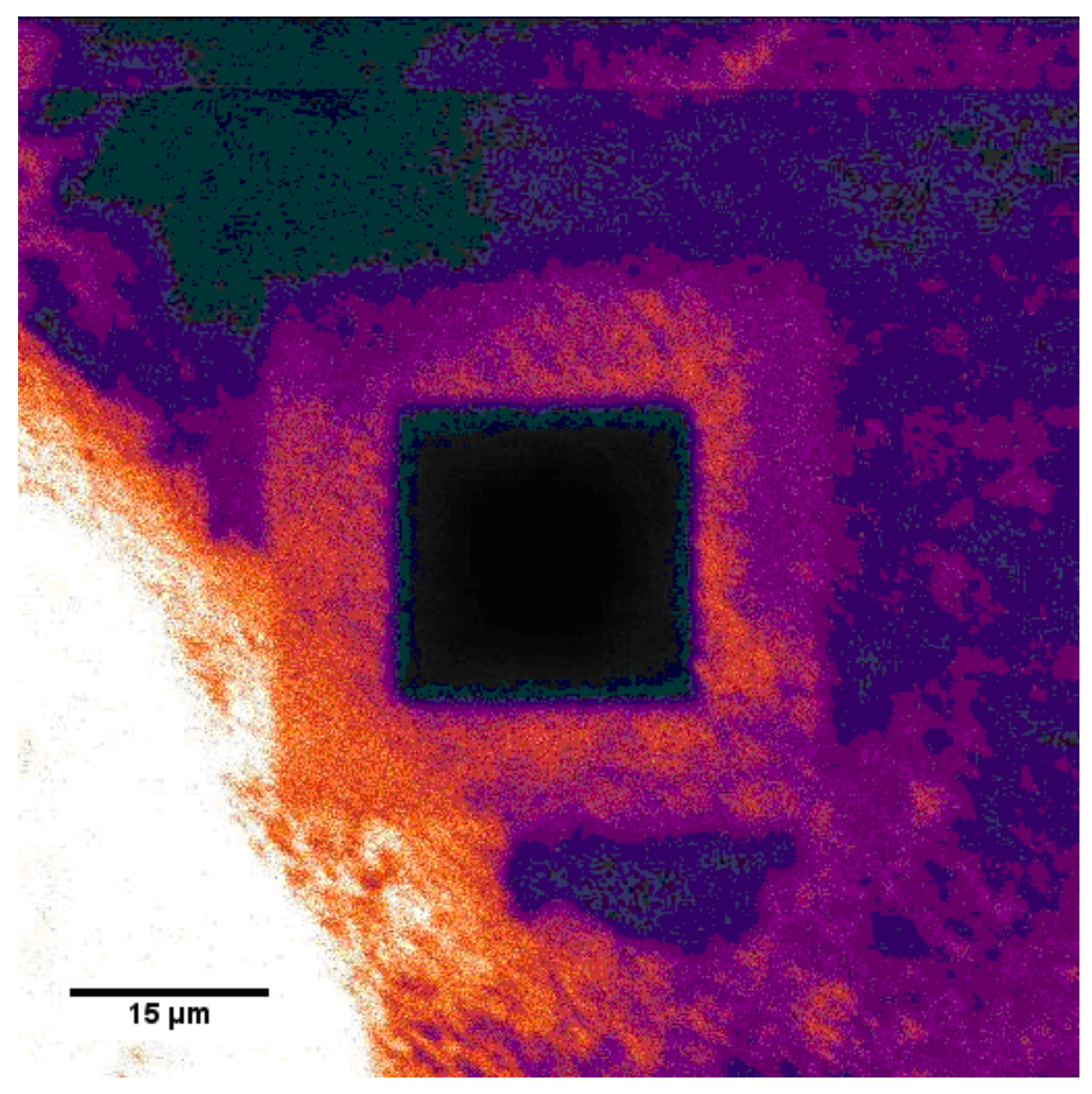

Figure 3.7: Fluorescence image of Rhodamine 6G demonstrating photobleaching.

\subsection{Optical Resolution}

The field of microscopy is primarily motivated by the imaging of extremely small features. Therefore, one of the most important characteristics of any microscopy system is resolution, the distance between two distinguishable points in an image. 
A microscope is incapable of revealing structure smaller than this limit. With our system functional, it was instructive to measure the resolution. This is done by examining a cross section of an image and measuring the distance between two sharp features - in this case, we used figure 3.5 and examined the cluster in the lower left corner to determine that the resolution was roughly $367 \mathrm{~nm}$, less than the illumination wavelength, as shown in figure 3.8.

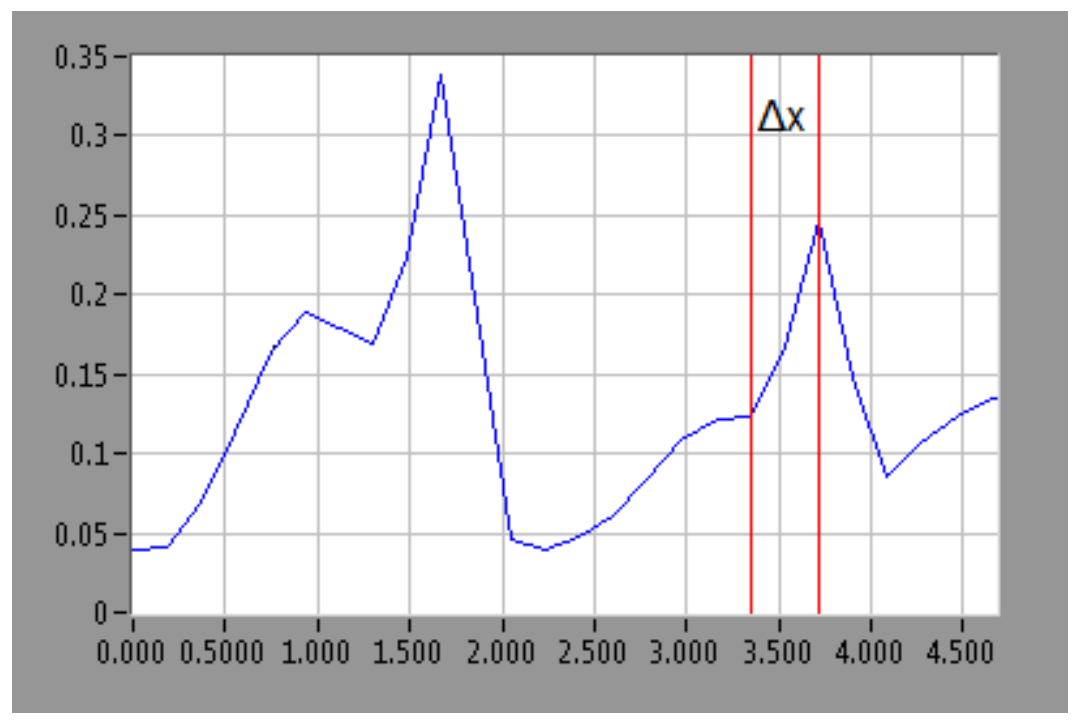

Figure 3.8: A cross section of magnetic glass reveals the resolution limit of our microscope to be less than $400 \mathrm{~nm}$. The $\mathrm{x}$ and y axes show lateral displacement in $\mu \mathrm{m}$ and relative light intensity in arbitrary units, respectively. The resolution is therefore given by $\Delta x$.

According to the Huygens-Fresnel principle, every point on a wavefront acts a source, radiating spherically. As light passes through a circular aperture, the spherical radiation leads to diffraction. Due to axial symmetry, the resulting diffraction pattern, known as the Airy pattern, takes the form of the function[35]

$$
y=\left(\frac{2 J_{1}(x)}{x}\right)^{2},
$$

where $J_{1}$ is the first order Bessel function of the first kind. 


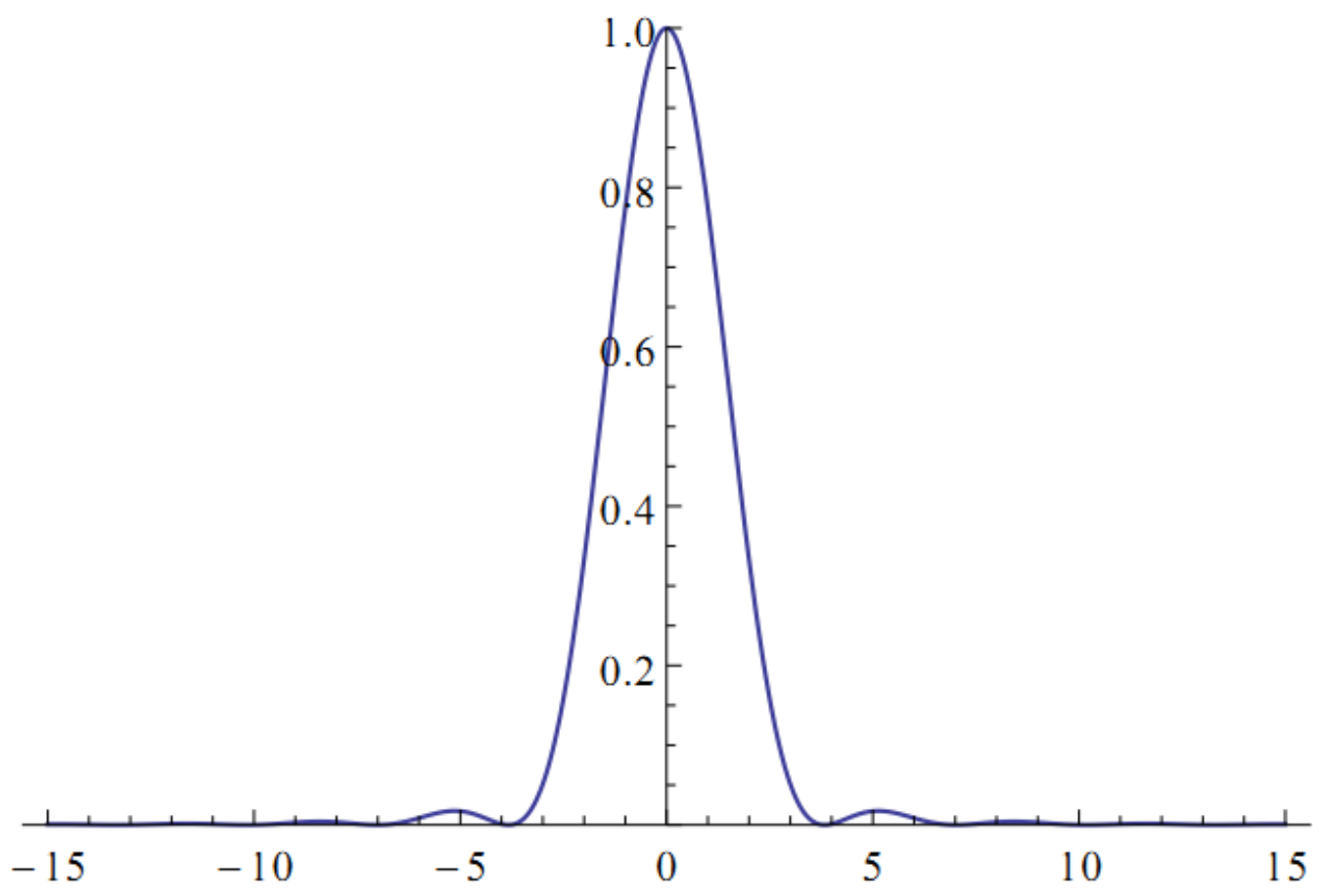

Figure 3.9: Airy pattern produced by propagation through a circular aperture.

The radius of the central peak of this disk is dependent on the wavelength of the light and the diameter of the aperture, and corresponds to the highest resolution obtainable with those parameters, given by[36]

$$
d=\frac{\lambda}{2(n \sin \alpha)},
$$

where $\lambda$ is the wavelength, $n$ is the index of refraction of the lens, and $\alpha$ is the halfangle of the light cone. The quantity $(n \sin \alpha)$ is defined as the numerical aperture of a lens, and is generally between 0.1 and $1.4[33]$.

For our system, using $514 \mathrm{~nm}$ light and an objective lens of $1.0 \mathrm{NA}$, the theoretical diffraction limit is equal to roughly $\lambda / 2$, or $257 \mathrm{~nm}$, indicating that the instrument in nearly diffraction limited. 


\section{Atomic Force Microscopy}

Atomic force microscopy is a type of scanning probe microscopy (SPM), a branch of microscopy in which the sample is imaged by scanning a physical probe over it. This also encompasses scanning tunneling microscopy (STM), electrostatic force microscopy (EFM), magnetic force microscopy (MFM), and near field scanning optical microscopy (NSOM), among many others. With the eventual goal of an NSOM Kerr microscope in mind, AFM capabilities are an absolute necessity.

\subsection{History of Scanning Probe Microscopy}

The field of SPM began in 1981, when Gerd Binnig and Heinrich Rohrer of IBM first

demonstrated the scanning tunneling microscope (STM)[25], work for which they would receive the Nobel Prize in Physics in 1986. In an STM, a bias is applied across a sharp metal tip (usually tungsten, platinum-iridium, or gold)[37] and a conducting sample. When the tip is brought into very close proximity with the sample, quantum tunneling effects are observed, according to the equation[37]

$$
I=\frac{4 \pi e}{\hbar} \int_{-\infty}^{+\infty}\left[f\left(E_{f}-e V+\epsilon\right)-f\left(E_{f}+\epsilon\right)\right] \rho_{S}\left(E_{f}-e V+\epsilon\right) \rho_{T}\left(E_{f}+\epsilon\right)|M|^{2} d \epsilon
$$

in which $f$ is the Fermi function, $E_{f}$ is the Fermi level, $e$ is electron charge, $V$ is potential, $\rho_{S}$ and $\rho_{T}$ are the densities of states of the sample and tip, and $\epsilon$ is the electron energy. $M$ is the tunnel matrix element[37], given by

$$
M=\frac{\hbar^{2}}{2 m} \int_{z=z_{0}}\left(\chi * \frac{\partial \psi}{\partial z}-\psi \frac{\partial \chi^{*}}{\partial z}\right) d S .
$$

Because the current is clearly dependent on the distance between the tip and the sample, $z$ in equation 4.2 , one may be used to monitor the other. In constant current mode, the tip is raster scanned over the sample and the current measurement is fed 
into an electronic feedback system. Using piezos, the microscope adjusts the height of the tip in an attempt to maintain constant current. The tip-sample distance, $z$, should therefore be constant, and a map of the piezo response yields a topographic map of the sample. STMs also function in constant height mode, where the tip is brought near the surface and scanned with no vertical movement. A map of the tunneling current is produced in this way, though it may not correlate as closely to topography as a constant current image[37].

In 1986, Binnig made another advancement in the field of SPM when he, along with Calvin Quate and Christoph Gerber, invented the atomic force microscope (AFM)[38]. One of the major limitations of STM was its reliance on a conductive sample. This requirement was removed with the advent of AFM, which uses atomic forces instead of tunneling current as the feedback mechanism.

\subsection{Basic Principles of AFM}

In AFM, a cantilever with a sharp tip, such as the one seen in figure 4.1, is scanned over the sample surface. A laser reflects from the cantilever into a segmented photodiode. As the tip interacts with the sample, the cantilever is deflected due to various atomic forces, including mechanical contact force, van der Waals forces, capillary forces, chemical bonding, electrostatic forces, magnetic forces, Casimir forces, solvation forces, and others[39]. This deflection is measured by monitoring the relative intensity on each segment of the photodiode. A conceptual diagram is shown in figure 4.2 .

The three main modes of operation for a typical AFM are contact mode, non contact mode, and tapping mode. In contact mode, the feedback loop works to maintain a constant force between the tip and the sample by using piezos to modulate the height of the tip. The piezo voltage then corresponds to sample topography. In non-contact mode, the cantilever is electrically driven near its resonance frequency. 


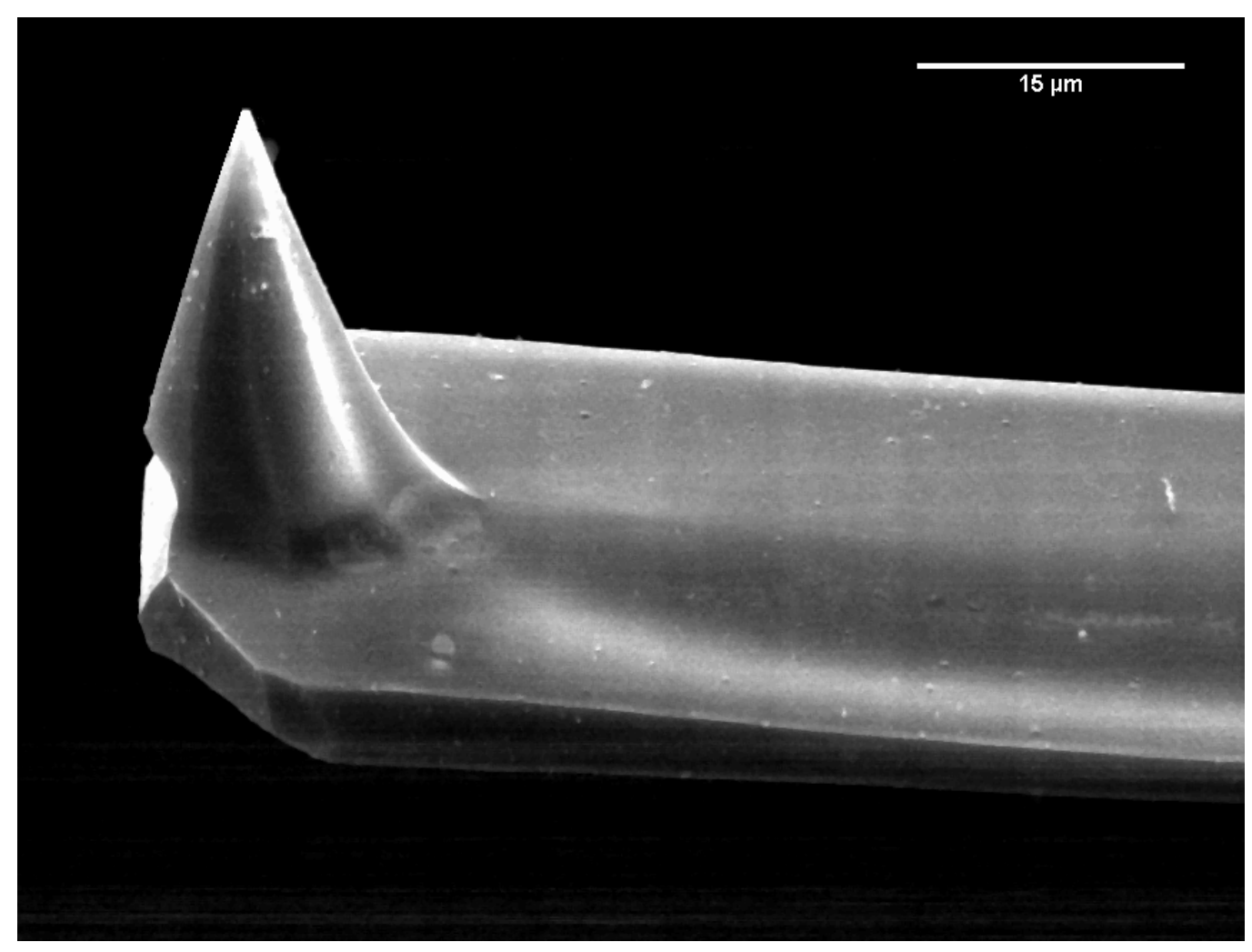

Figure 4.1: SEM image of AFM tip.

As the tip nears the sample surface, atomic forces interact with the cantilever and act to dampen its oscillation. The amplitude or phase then serves as the feedback signal and the piezo voltage is mapped to reveal topography. In tapping mode, the tip is driven mechanically to oscillate near its resonant frequency. The amplitude of the oscillation decreases when the tip is near the surface, and the feedback system works to maintain a constant amplitude[40].

One of the greatest complications of traditional AFM use is the alignment of the feedback laser. A variation of AFM known as shear force microscopy (SFM) removes this complication by replacing the cantilever based feedback system with a quartz tuning fork. The tip is mounted to one tine of the fork, which is driven near its resonance frequency for non-contact mode imaging[41]. 


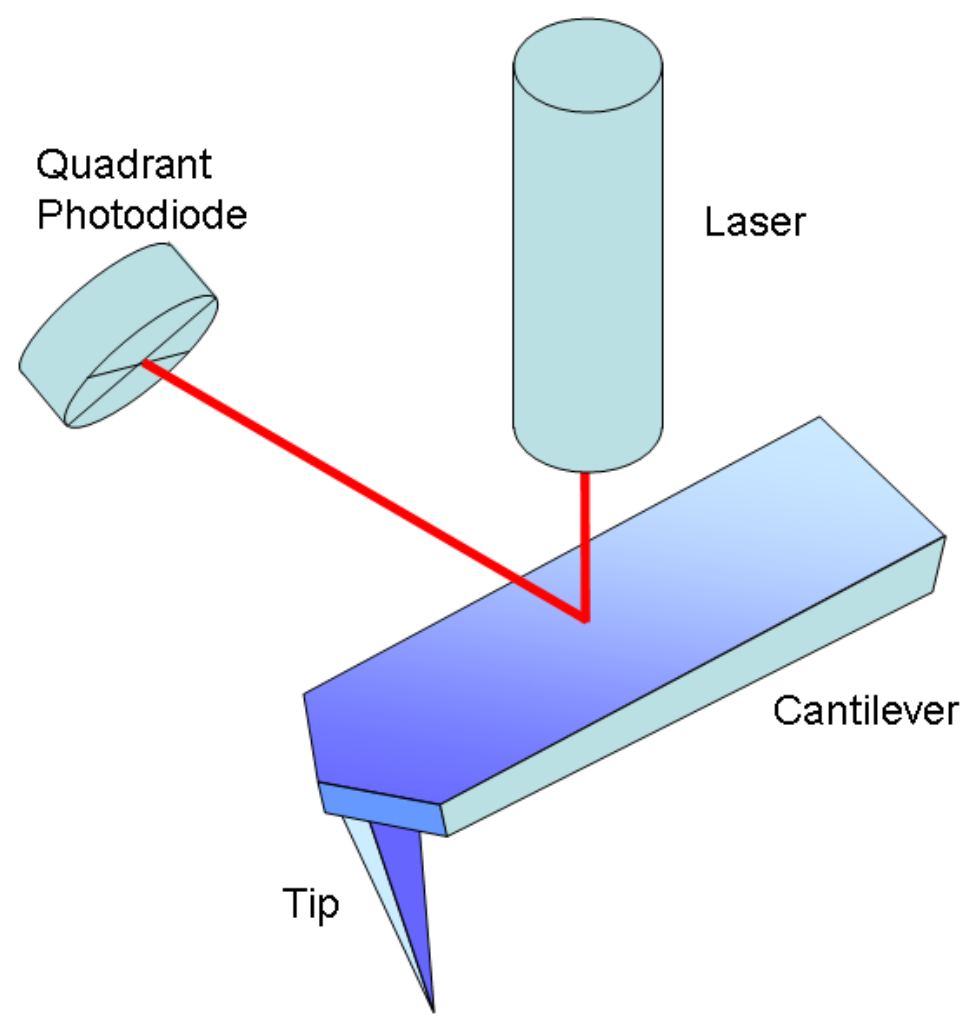

Figure 4.2: Conceptual diagram of an AFM.

\subsection{Design of AFM}

The optical microscope, the technical details of which were described in section 3.2, was designed with forethought given to the necessity of integration of AFM capabilities. The custom base plate, which serves as the ceiling and sample holder of the optical microscope, functions as the base of the AFM, leaving the two systems integrated yet independent. A pocket milled into the base plate accommodates the $\mathrm{x}-\mathrm{y}$ piezo scan stage (Physik Instrumente 733.2CL), the details of which were discussed earlier in section 3.4 .

Mounted on the base plate is a stable backplane assembly, consisting of a thick plate and three braces, all custom designed in SolidWorks and machined from cast iron. The sole function of the backplane assembly is to position the AFM scan head 
such that the tip is suspended over the sample surface. Mounted to the back of the scan head is a dovetail (Thorlabs XT66D3) which nests in custom rails mounted to a $50 \mathrm{~mm}$ translation stage (Thorlabs LNR50M with drive removed). The translation stage is mounted to the backplane, and the $\mathrm{z}$ height is controlled by a stepper motor (Oriental Motors PK243B1A-SG36) and lead screw (Universal Thread) assembly.

The primary design complication of the backplane was the three-dimensional positioning of the AFM tip. The $\mathrm{x}$ and $\mathrm{y}$ dimensions were constrained by the necessity for the AFM tip, and therefore the center of the scan head, to be perfectly centered over the objective lens, thereby making simultaneous optical and AFM imaging possible. The $\mathrm{z}$ dimension was less critical due to the inherent flexibility afforded by the 50 $\mathrm{mm}$ translation stage, but in general, it was desirable that the tip be near the surface when the stage is positioned midway through its range of travel.

The realization of these requirements was greatly simplified by the CAD design process undertaken in SolidWorks. Separate subassemblies were constructed for the scan head, backplane, and optical microscope. By making the necessary mates within SolidWorks, such as constraining the dovetail to the dovetail rails, the bottom of the backplane to the top surface of the base plate, etc., we were left with just three degrees of freedom - the $\mathrm{x}$ and $\mathrm{y}$ positions of the backplane and the $\mathrm{z}$ position of the scan head. The subassemblies were then moved to appropriate locations, revealing the optimal positions for the mounting holes. For added flexibility, slots were machined in the backplane braces, allowing $1 / 4$ " of travel in the y position of the backplane.

The most critical component of the AFM is the scan head, which houses the piezo stack, the tuning fork, a CCD camera for tip viewing, and the circuit boards for signal amplification. The original scan head design also included a low power laser for tip illumination, which proved unnecessary.

The piezo stack consists of two piezo tubes (Boston Piezo-Optics PZT-5A and 
PZT-5A Segmented) separated by an insulative glass-ceramic ring (Corning MACOR) to which each is epoxied. The unsegmented tube provides fine control of $\mathrm{z}$ for topographic scanning, while the segmented tube allows for fine adjustment of $\mathrm{x}$ and $y$ for precise positioning of the tip relative to the objective lens. A MACOR ring is epoxied to the exterior of the upper tube and held in a flexure mount (Newport MFM-075) for coarse positioning in $\mathrm{x}$ and $\mathrm{y}$. A final MACOR piece is epoxied between the bottom of the stack and an aluminum mounting ring. A preamplifier board for the tuning fork holder is epoxied to an aluminum holder, which is screwed to the aluminum ring with an additional plastic ring between to reduce mechanical coupling. The tuning fork is fixed securely in the holder piece with a set screw and soldered directly to the preamplifier board. Figure 4.3 shows the complete scan head with the piezo stack in place.

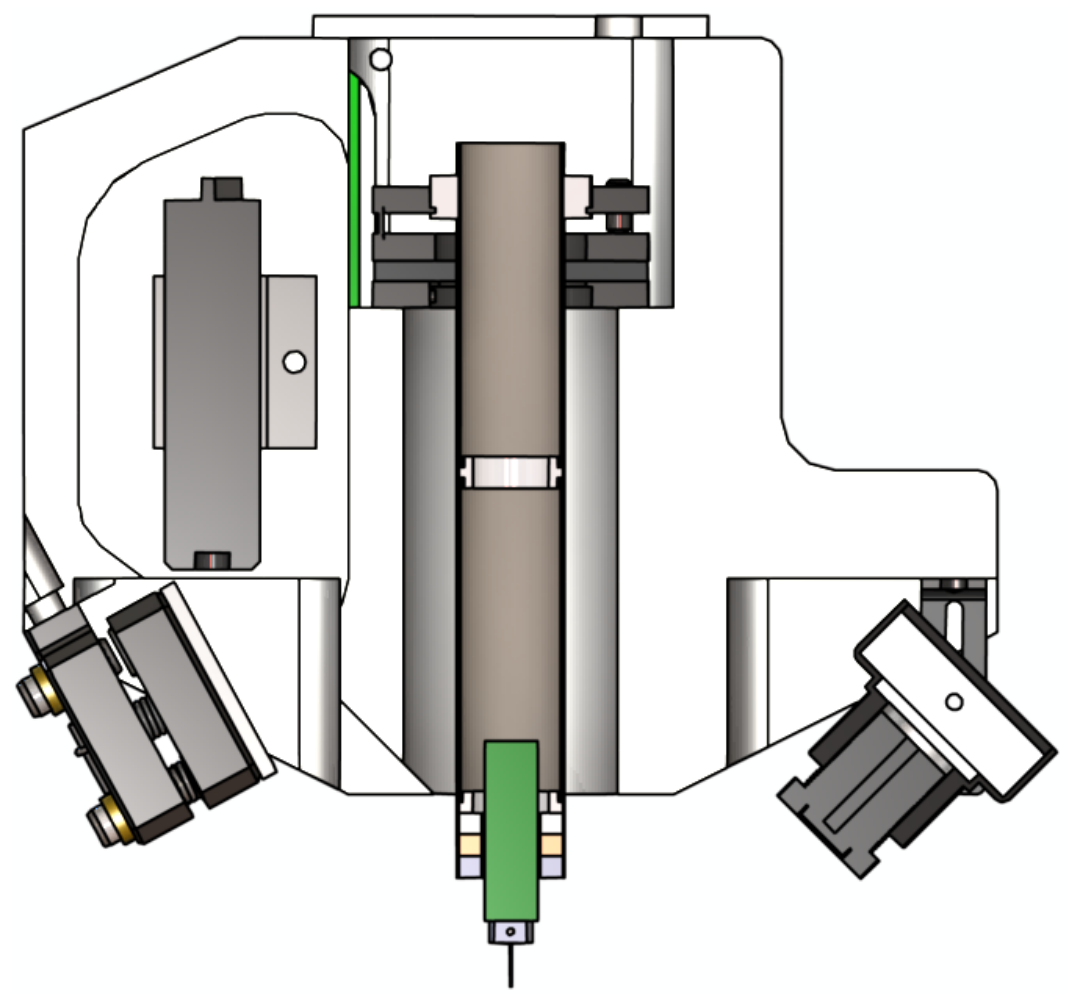

Figure 4.3: SolidWorks cross-section of scan head reveals piezo stack and tuning fork. 
The complete microscope, including the backplane assembly, scan head, and breakout box, is shown in figure 4.4 .

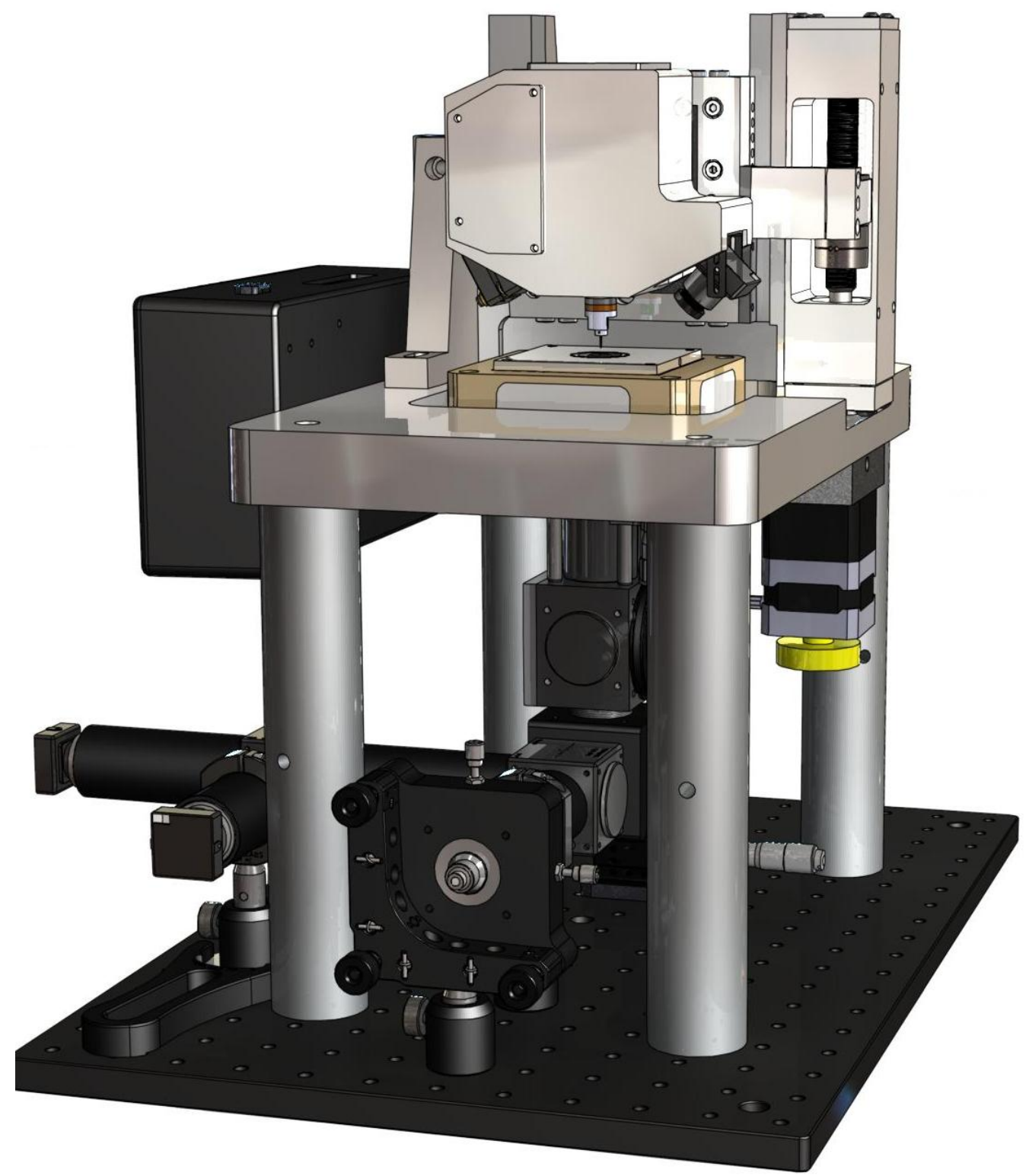

Figure 4.4: SolidWorks model of complete atomic force inverted optical microscope, including backplane assembly, scan head, and breakout box.

The base plate was machined on a CNC mill using the SolidWorks drawing as an input (as mentioned in section 3.2). All other parts were fabricated using a standard 
milling machine out of cast iron, aluminum, and MACOR. Once assembled, the microscope was placed in a light proof enclosure on a floating optical table to shield it from external fields and vibration[40]. The end result is a functional atomic force inverted optical microscope, as seen in figure 4.5.

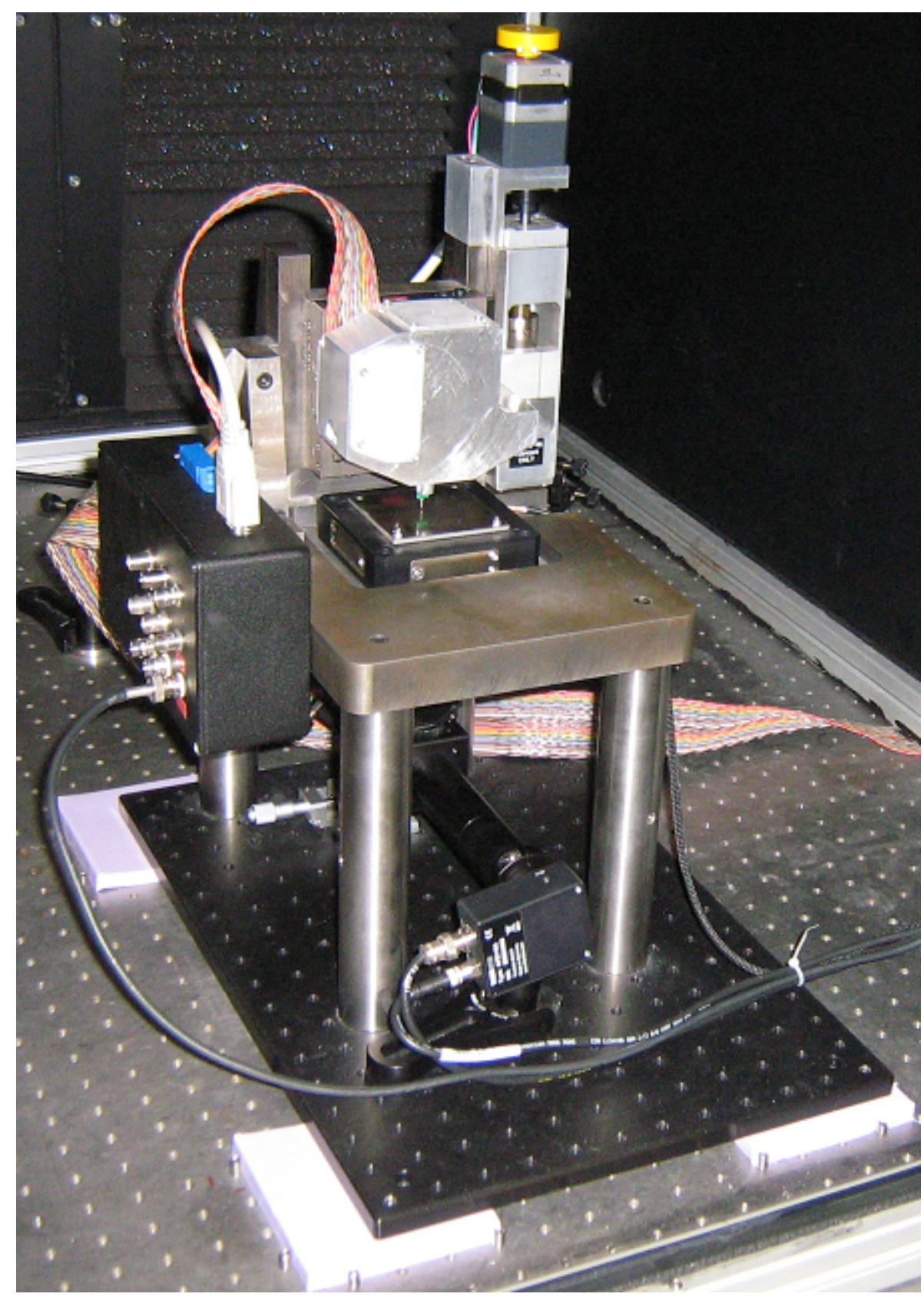

Figure 4.5: Photograph of functional atomic force microscope. 


\subsection{AFM Electronics}

The analog requirements for the microscope were supplied by custom printed circuit boards (PCBs) designed in the software package PCB123, v.2 (Sunstone Circuits) as part of the open source ANSOM Project[42], developed in our lab. These were developed so as to be as modular as possible. In addition to the main controller board, there are boards for phase detection, driving of the piezos, control of the stepper motor, breakout from the data acquisition (DAQ) card, and amplification of the tuning fork signal.

The controller board interfaces directly with the DAQ, fulfilling the role of routing the digital and analog signals through the system. It also contains the electronics for control of the scan and offset (as discussed in section 3.4), driving of the tuning fork, and amplitude detection.

The tuning fork is driven by a high resolution sine wave near its resonance of $2^{15}$ $\mathrm{Hz}$ by a direct digital synthesis (DDS) integrated circuit (Analog Devices AD9835). This signal is used as a reference oscillator for phase detection as well as the drive signal for the tuning fork. The amplitude detection circuitry consists of an RMS-toDC converter (Analog Devices AD637) and a 2-pole Sallen-Key filter[31]. For phase detection, a high speed (4.5 ns) comparator (Linear Technologies LT1712) compares the reference signal to the response of the tuning fork to detect phase differences[31].

The piezo driver board consists of two operational amplifiers (Apex P88) arranged in a bridge circuit, with $170 \mathrm{~V}$ supplied by two $155 \mathrm{~V}$ DC power supplies (International Power IHB155-0.12). Three such boards were created so the tip position in each axis can be controlled independently[31].

The controller board, phase detection board, and piezo driver boards are housed, along with the external power supplies, in an external controller box. An additional electronics enclosure (Hammond $1590 \mathrm{BBK}$ ) was mounted to the side of the base plate 
to house a breakout board for the FPGA card and a stepper motor control board[31].

The tuning fork board, which serves as a preamplifier for the response signal of the tuning fork, and the scan head board, which further amplifies the signal, are located within the scan head itself. The close physical proximity to the tuning fork serves to reduce the additional noise and capacitance inherent to signals transmitted over a large distance.

\subsection{Control of AFM}

The control systems of the AFM were alo obtained from the ANSOM Project[42], and take the form of a comprehensive LabVIEW project. The project is developed around a National Instruments data acquisition (DAQ) card (National Instrument PCI-7852R) with a field-programmable gate array (FPGA) microprocessor. The logic necessary to control the operation of the microscope is programmed in LabVIEW and compiled directly to the FPGA.

The primary algorithms of the microscope are those that control scanning and sample surface approach, as well as the PID (proportional-integral-derivative) feedback algorithm. The scanning algorithm is discussed in section 3.4. The ANSOM Project also includes a graphical user interface (GUI) with tools for control of the oscillation signal, z piezo, stepper motor, cameras, scanning, and image processing.

The approach algorithm controls the z piezo as well as the stepper motor in order to bring the tip within $500 \mathrm{~nm}[31]$ of the surface without damaging either. While monitoring the phase feedback signal, the z piezo is extended to its limit. If the tip does not encounter the surface, which would be indicated by a sudden change in the feedback signal, the tip is retracted and the motor takes one step towards the surface. This process continues until the surface is located, at which point control of the $\mathrm{z}$ piezo is transferred to the PID algorithm[31].

The PID algorithm attempts to maintain a setpoint in the feedback signal. As the 
system scans over topographic features, the PID loop serves to maintain a constant interaction force between the tip and the sample. This is accomplished by reacting to changes in the feedback signal. As the tip approaches a tall feature, the interaction force increases, and the PID loop signals the $\mathrm{z}$ piezo to retract; as the tip approaches a low feature, the interaction force decreases, and the PID loop signals the $\mathrm{z}$ piezo to extend[31]. The signal sent to the z piezo is mapped to reveal the sample topography.

\subsection{AFM Results}

Upon successful development and integration of the various systems necessary for operation of the AFM, investigation was undertaken on an AFM calibration grid (Nanosurf) consisting of square pillars with $119 \mathrm{~nm} \mathrm{z}$ height and $10 \mu \mathrm{m}$ periodicity[43]. The phase and topographic images are shown in figures 4.6 and 4.7, respectively.

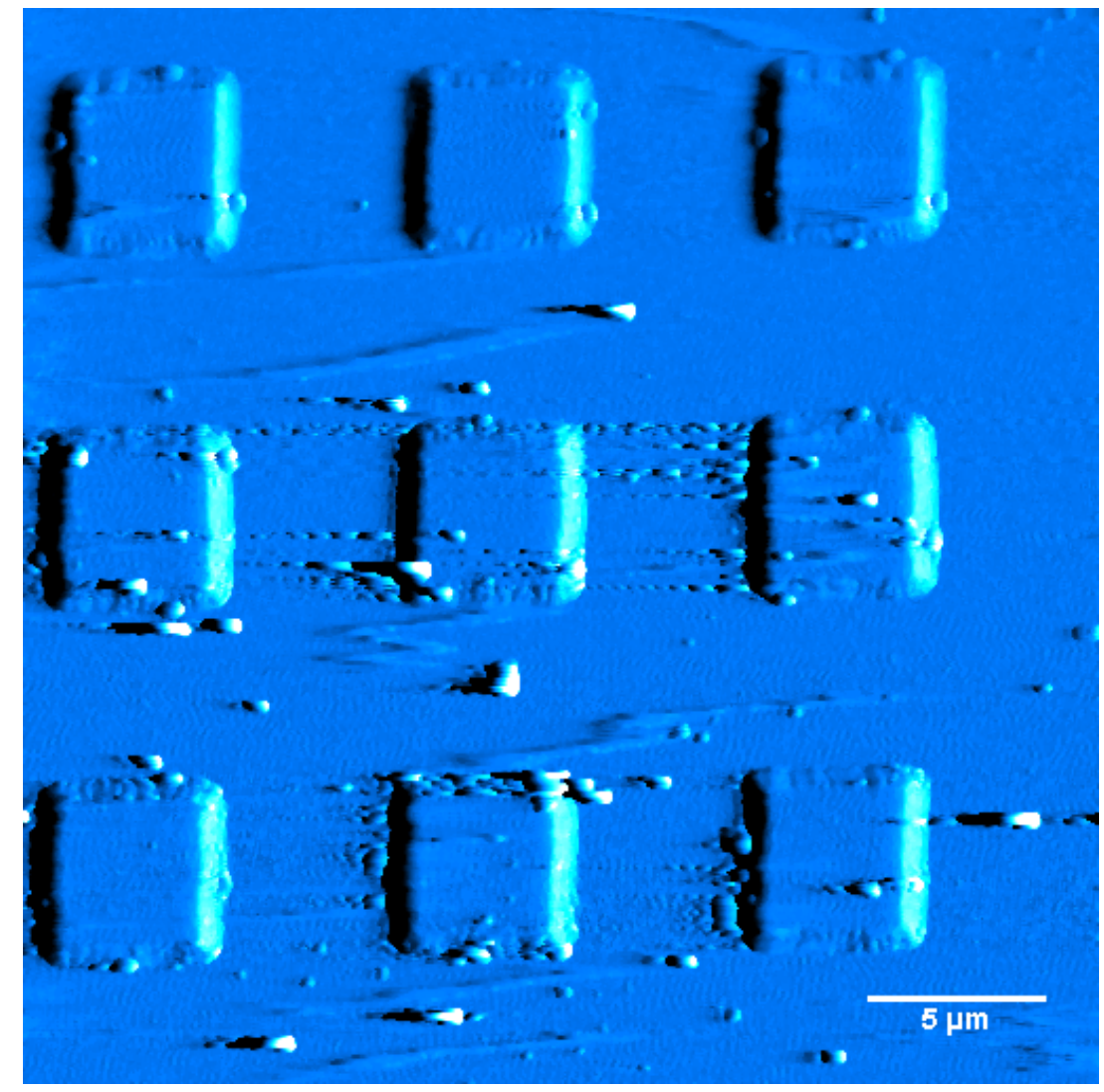

Figure 4.6: Phase image of AFM calibration grid. 


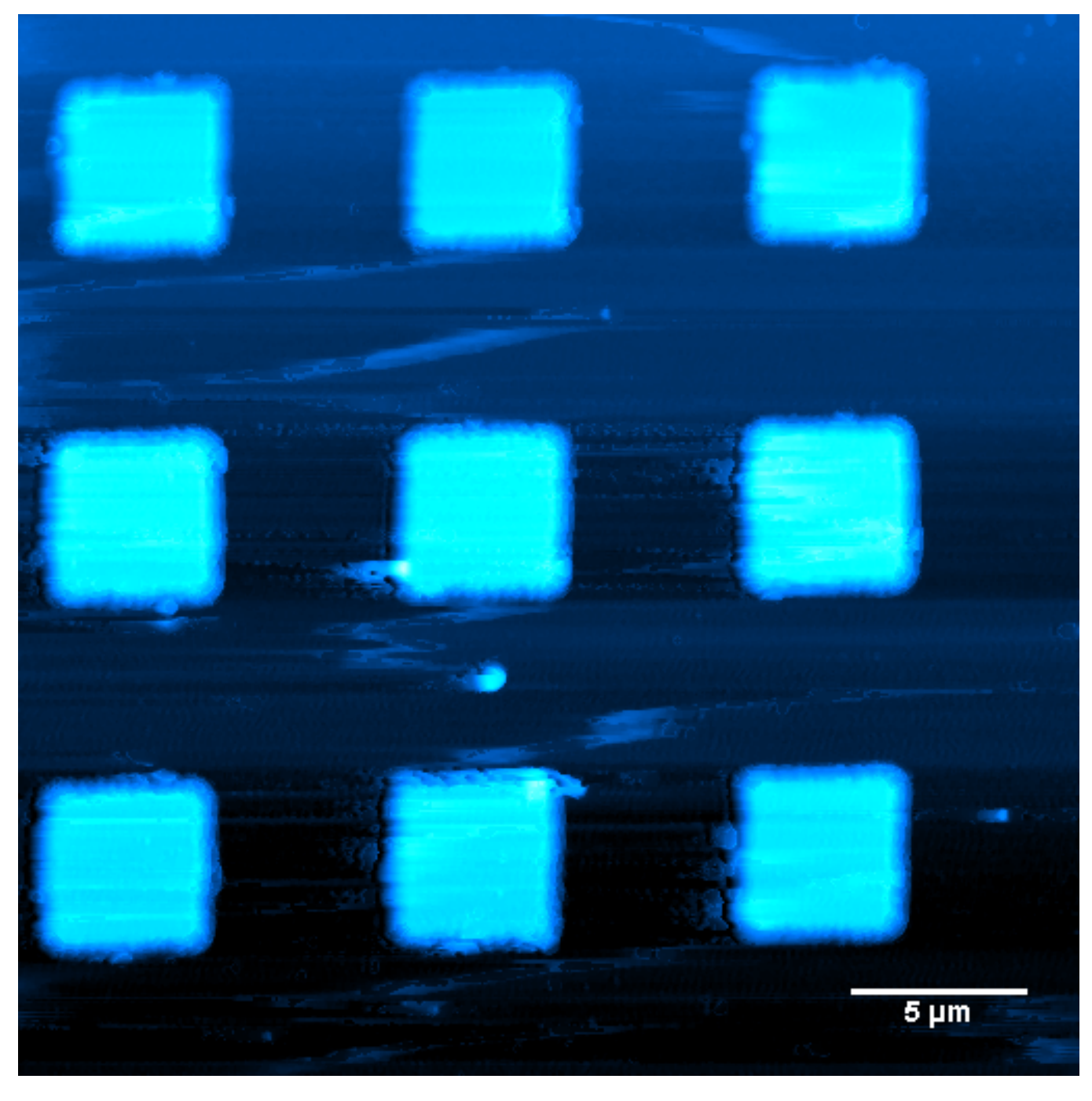

Figure 4.7: Topographic image of AFM calibration grid.

These initial scans evince the utility of the instrument. The lateral resolution is constrained only by the aspect ratio of the tip, and can be measured by monitoring the feedback response of the scan bed in closed loop mode. Successful topographic scanning demonstrates the ability of the microscope to reproducibly approach and scan over the sample surface without damage to the probe tip, capabilities that will be essential to the continuation of the project as we adapt the system to fiber-based NSOM microscopy. 


\section{Magneto-Optic Kerr Effect}

Kerr microscopy is made possible by the magneto-optical Kerr effect (MOKE), a phenomenon in which the polarization of light reflected in the presence of a magnetic field undergoes a shift in both ellipticity and rotation[44]. By detecting this change in polarization, it is possible to image magnetic orientation with an optical microscope. The effect is demonstrated in figure 5.1.

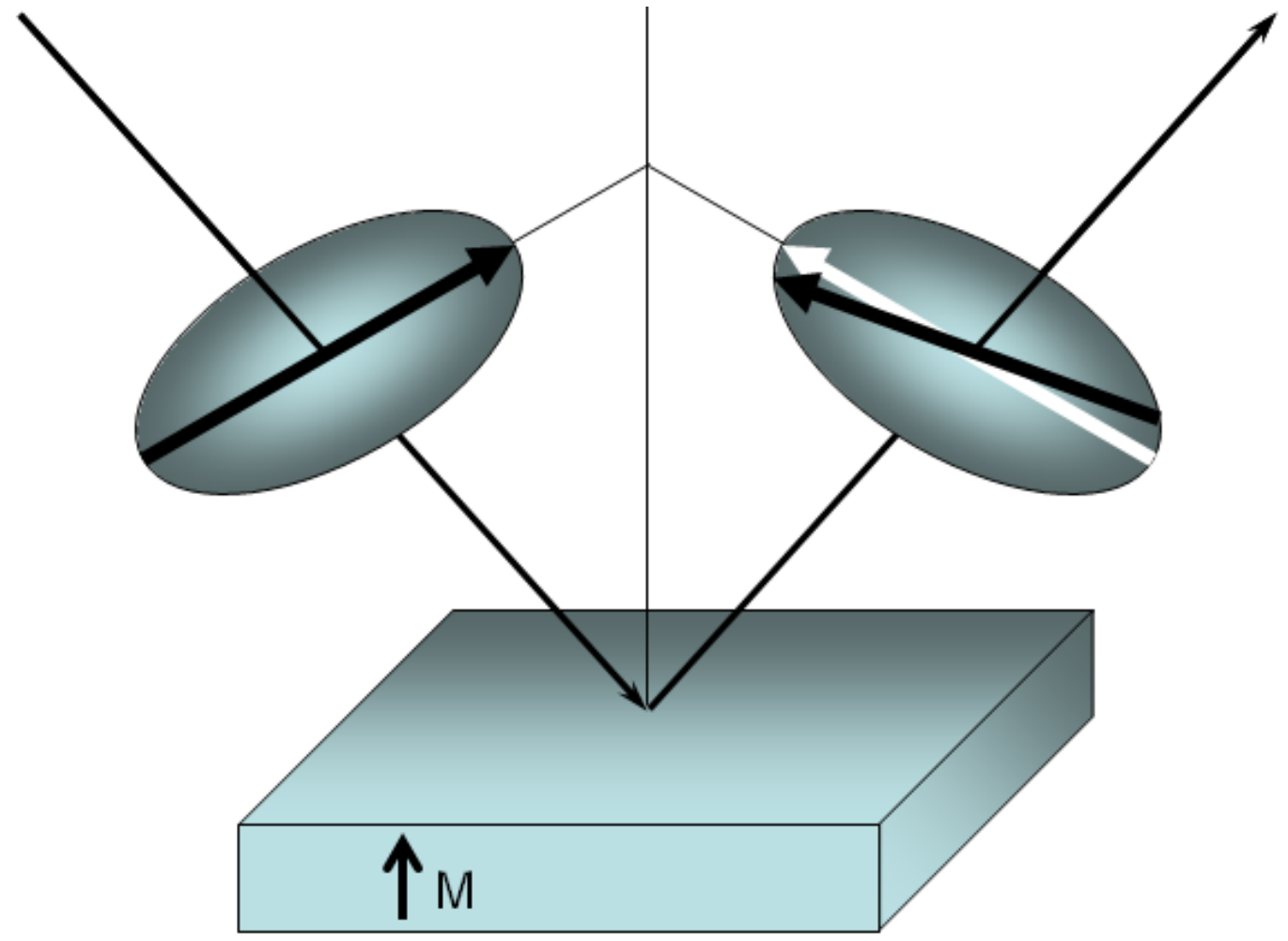

Figure 5.1: Illustration of polar MOKE, in which the magnetization is normal to the surface. The disks represent the plane of polarization in the direction of the black arrows.

\subsection{History of Magneto Optics}

The field of magneto-optics began in 1846 when Michael Faraday published his paper

"On the Magnetic Affection of Light". In it, he described the interaction of light and 
magnetism: "When a ray of polarized light and lines of magnetic force pass simultaneously and parallel to each other through a transparent (medium), the ray is rotated according to a simple law of action... [45]" This interaction, appropriately enough, came to be known as the Faraday effect. This effect has been used for the measurement of magnetic fields[46], characterization of electron spins in semiconductors[47], and many other purposes. However, it is perhaps most significant as the first empirical evidence for the relationship between light and magnetism, and therefore the beginning of the entire field of magneto-optics.

It was John Kerr who, in 1877, made the logical leap to extend Faraday's principle to reflected light[48]. Kerr's apparatus was fairly crude by modern standards, but it was nevertheless sufficient to demonstrate the effect that now bears his name. The light from a paraffin candle was polarized using a Nicol prism and reflected from the highly polished surface of a horseshoe electromagnet. The magnet was powered by six Grove cell batteries, providing roughly 12 volts DC [49], and consisted of an iron core solenoid two inches in diameter with about 400 turns of wire. A wedge of iron was also placed above the surface to concentrate the field. A second Nicol was placed in the path of the reflected beam and oriented so as to extinguish the reflection in the absence of a magnetic field.

As he expected, Kerr found that by applying power to the electromagnet, the effect on the polarization was sufficient to make the light visible through the second prism. He further found that with the field applied, he was unable to extinguish the beam regardless of the orientation of the second prism, indicating that the polarization had changed in ellipticity as well as angle. Kerr went on to describe twelve experiments he performed, further contributing to our understanding of the complex interaction between light and magnetism. 


\subsection{Mathematical Background on the Propagation of Light}

Classical electrodynamics is underscored by a set of four partial differential equations, including Gauss' law, Ampère's law, Faraday's law of induction, and Gauss' law for magnetism[50]:

$$
\begin{array}{r}
\nabla \cdot \mathbf{D}=\rho_{f}, \\
\nabla \times \mathbf{H}=\mathbf{J}_{f}+\frac{\partial \mathbf{D}}{\partial t}, \\
\nabla \times \mathbf{E}=-\frac{\partial \mathbf{B}}{\partial t}, \\
\nabla \cdot \mathbf{B}=0,
\end{array}
$$

in which $\rho_{f}$ is the free charge density, $\mathbf{J}_{\mathbf{f}}$ is the free current density, and $\mathbf{E}, \mathbf{D}, \mathbf{B}$, and $\mathbf{H}$ represent the electric, displacement, magnetic, and magnetizing fields, respectively. These are known collectively as Maxwell's equations, and along with the Lorentz force law, serve as the foundation for nearly all of classical electrodynamics. They are therefore also crucial to the fields of optics and electronics.

We begin with the familiar form of Maxwell's equations in the absence of free charges and currents, so that $\mathbf{J}_{\mathbf{f}}$ and $\rho_{f}$ go to zero and the above equations become

$$
\begin{array}{r}
\nabla \cdot \mathbf{D}=0, \\
\nabla \times \mathbf{H}=\frac{\partial \mathbf{D}}{\partial t}, \\
\nabla \times \mathbf{E}=-\frac{\partial \mathbf{B}}{\partial t}, \\
\nabla \cdot \mathbf{B}=0 .
\end{array}
$$

Following the procedure of Mansuripur, we have[5]

$$
\underline{u}=\left(u_{x}, u_{y}, u_{z}\right),
$$


and

$$
\underline{\underline{u}}=\left[\begin{array}{ccc}
0 & -u_{z} & u_{y} \\
u_{z} & 0 & -u_{x} \\
-u_{y} & u_{x} & 0
\end{array}\right],
$$

along with the recognition that

$$
\mathbf{D}=\epsilon_{0} \epsilon \mathbf{E}
$$

and

$$
\mathbf{B}=\mu_{0} \mathbf{H}
$$

where $\epsilon_{0}$ is the permittivity of free space, $\underset{\sim}{\epsilon}$ is the dielectric tensor of the propagation medium, and $\mu_{0}$ is the permeability of free space. We may express Maxwell's equations in a more convenient matrix notation as

$$
\begin{array}{r}
\underline{\nabla} \underset{\sim}{\epsilon} \underline{E}^{T}=0, \\
\underline{\nabla} \underline{H}^{T}=-\mathrm{i} \omega \epsilon_{0} \in \underline{E}^{T}, \\
\underline{\nabla} \underline{E}^{T}=\mathrm{i} \omega \mu_{0} \underline{H}^{T}, \\
\underline{\nabla} \underline{H}^{T}=0 .
\end{array}
$$

We then proceed to normalize Maxwell's equations by the impedance of free space,

$$
Z_{0}=\sqrt{\mu_{0} / \epsilon_{0}}
$$


and obtain

$$
\begin{array}{r}
\underline{\nabla} \underset{\sim}{\hat{\epsilon} \underline{\hat{E}}^{T}=0,} \\
\underline{\underline{\nabla}} \underline{H}^{T}=-\mathrm{i} \omega \sqrt{\frac{\mu_{0}}{\epsilon_{0}}} \epsilon_{0} \underset{\sim}{\hat{E}^{T}}=-\mathrm{i} k_{0} \underset{\sim}{\epsilon} \hat{E}^{T}, \\
\underline{\underline{\nabla}} \underline{\hat{E}}^{T}=\mathrm{i} \omega \sqrt{\frac{\mu_{0}}{\epsilon_{0}}} \mu_{0} \underline{H}^{T}=\mathrm{i} k_{0} \underline{H}^{T}, \\
\underline{\nabla} \underline{H}^{T}=0,
\end{array}
$$

where we have used $\underline{\hat{E}}^{T}$ to indicate the normalized electric field vector and introduced $k_{0}$, the propagation constant of free space:

$$
k_{0}=\sqrt{\varepsilon_{0} \mu_{0}} \omega
$$

If we have a plane wave defined by

$$
\begin{aligned}
\mathbf{E}(\mathbf{r}, t) & =\mathbf{E}_{0} \exp [\mathrm{i}(\mathbf{k} \cdot \mathbf{r}-\omega t)], \\
\mathbf{H}(\mathbf{r}, t) & =\mathbf{H}_{0} \exp [\mathrm{i}(\mathbf{k} \cdot \mathbf{r}-\omega t)],
\end{aligned}
$$

then equations 5.9a-d become

$$
\begin{array}{r}
\underline{k \in} \in \underline{\hat{E}}_{0}^{T}=0, \\
\underline{\underline{k}} H_{0}^{T}=-k_{0} \epsilon \underline{\hat{E}}_{0}^{T}, \\
\underline{\underline{k}}_{\hat{E}_{0}}^{T}=k_{0} \underline{H}_{0}^{T}, \\
\underline{k} \underline{H}_{0}^{T}=0 .
\end{array}
$$

Multiplying equation $5.12 \mathrm{~b}$ by $\underline{\underline{k}}$ and substituting equation $5.12 \mathrm{c}$ into the right hand 
side yields the Helmholtz equation

$$
\left[\left(\frac{k}{k_{0}}\right)^{2}+\underset{\epsilon}{\epsilon}\right] \hat{E}_{0}^{T}=0,
$$

which has a nontrivial solution when

$$
\left|\left(\frac{k}{k_{0}}\right)^{2}+\epsilon\right|=0 .
$$

\subsection{Light in a Medium}

Still following the procedure of Mansuripur, we consider a plane wave propagating in an isotropic medium, we may take the dielectric tensor to be $\underset{\sim}{\epsilon}=\epsilon \mathbf{I}$ where $\mathbf{I}$ is a $3 \mathrm{x}$ 3 identity matrix. Equation 5.14 then becomes

$$
\left|\frac{1}{k_{0}^{2}}\left[\begin{array}{ccc}
-k_{y}^{2}-k_{z}^{2} & k_{x} k_{y} & k_{x} k_{z} \\
k_{x} k_{y} & -k_{x}^{2}-k_{z}^{2} & k_{y} k_{z} \\
k_{x} k_{z} & k_{y} k_{z} & -k_{x}^{2}-k_{y}^{2}
\end{array}\right]+\left[\begin{array}{ccc}
\epsilon & 0 & 0 \\
0 & \epsilon & 0 \\
0 & 0 & \epsilon
\end{array}\right]\right|=0
$$

and solving for the determinant yields

$$
k_{x}^{2}+k_{y}^{2}+k_{z}^{2}=k_{0}^{2} \epsilon .
$$

If we confine the wave vector $\mathbf{k}$ to the $\mathrm{y}-\mathrm{z}$ plane, we may proceed with the assumptions that $k_{x}=0$ and $k_{y}=k_{0} \sin \theta$. Equation 5.16 then becomes

$$
k_{z}=k_{0} \sqrt{\epsilon-\sin ^{2} \theta},
$$


and the Helmholtz equation 5.13 is therefore

$$
\left[\begin{array}{ccc}
0 & 0 & 0 \\
0 & \sin ^{2} \theta & \pm \sin \theta \sqrt{\epsilon-\sin ^{2} \theta} \\
0 & \pm \sin \theta \sqrt{\epsilon-\sin ^{2} \theta} & \epsilon-\sin ^{2} \theta
\end{array}\right]\left[\begin{array}{c}
\hat{E}_{x} \\
\hat{E}_{y} \\
\hat{E}_{z}
\end{array}\right]=0 .
$$

This matrix gives three equations, but one is trivial, and the other two are equivalent:

$$
\hat{E}_{z}= \pm \frac{\sin \theta}{\sqrt{\epsilon-\sin ^{2} \theta}} \hat{E}_{y}
$$

Rearrangement of the equation $5.12 \mathrm{~b}$ yields

$$
\underline{H}_{0}^{T}=\left(\underline{\underline{k}} / k_{0}\right) \underline{\hat{E}}_{0}^{T},
$$

which can be used define a relationship between the electric and magnetic fields. Using equation 5.17 along with the values we chose for $k_{x}$ and $k_{y}$, equation 5.20 becomes

$$
\left[\begin{array}{c}
H_{x} \\
H_{y} \\
H_{z}
\end{array}\right]=\left[\begin{array}{ccc}
0 & \mp \sqrt{\epsilon-\sin ^{2} \theta} & \sin \theta \\
\pm \sqrt{\epsilon-\sin ^{2} \theta} & 0 & 0 \\
-\sin \theta & 0 & 0
\end{array}\right]\left[\begin{array}{c}
\hat{E}_{x} \\
\hat{E}_{y} \\
\hat{E}_{z}
\end{array}\right] .
$$

Substituting equation 5.19 into the first of these equations gives

$$
\begin{array}{r}
H_{x}=\mp \frac{\epsilon}{\sqrt{\epsilon-\sin ^{2} \theta}} \hat{E}_{y}, \\
H_{y}= \pm \sqrt{\epsilon-\sin ^{2} \theta} \hat{E}_{x}, \\
H_{z}=-\sin \theta \hat{E}_{x} .
\end{array}
$$


The third equation is unnecessary because $H_{z}$ is rarely of interest [5] and can be defined in terms of $H_{y}$ if necessary. We are therefore left with the following relationship between the $\vec{E}$ and $\vec{H}$ fields for a plane wave propagating in an isotropic medium:

$$
\left[\begin{array}{l}
H_{x} \\
H_{y}
\end{array}\right]= \pm\left[\begin{array}{cc}
0 & -\frac{\epsilon}{\sqrt{\epsilon-\sin ^{2} \theta}} \\
\sqrt{\epsilon-\sin ^{2} \theta} & 0
\end{array}\right]\left[\begin{array}{l}
\hat{E}_{x} \\
\hat{E}_{y}
\end{array}\right]
$$

\subsection{Mathematical Formulation of MOKE}

When the principles of the preceding section are applied to an anisotropic medium, the math becomes more complicated. The dielectric tensor can no longer be represented by an identity matrix. We begin by considering the case of a homogeneous medium under the influence of a magnetic field oriented along the z-axis. The dielectric tensor is given by:

$$
\underset{\sim}{\epsilon}=\left[\begin{array}{ccc}
\epsilon & \epsilon^{\prime} & 0 \\
-\epsilon^{\prime} & \epsilon & 0 \\
0 & 0 & \epsilon
\end{array}\right]
$$

And with $k_{x}=0$ and $k_{y}=k_{0} \sin \theta$, equation 5.14 becomes

$$
\left|\left[\begin{array}{ccc}
\epsilon-\sin ^{2} \theta-\frac{k_{z}^{2}}{k_{0}^{2}} & \epsilon^{\prime} & 0 \\
-\epsilon^{\prime} & \epsilon-\frac{k_{z}^{2}}{k_{0}^{2}} & \frac{k_{z}}{k_{0}} \sin \theta \\
0 & \frac{k_{z}}{k_{0}} \sin \theta & \epsilon-\sin ^{2} \theta
\end{array}\right]\right|=0 .
$$

Some algebra yields the characteristic equation

$$
\left(\frac{k_{z}}{k_{0}}\right)^{4}-2\left(\epsilon-\sin ^{2} \theta\right)\left(\frac{k_{z}}{k_{0}}\right)^{2}+\left[\left(\epsilon-\sin ^{2} \theta\right)^{2}-\epsilon^{\prime 2}\left(\frac{\sin ^{2} \theta}{\epsilon}-1\right)\right]=0,
$$


and an application of the quadratic formula yields the solutions:

$$
\begin{aligned}
& k_{z 1}= \pm k_{0} \sqrt{\left(\varepsilon-\sin ^{2} \theta\right)+\varepsilon^{\prime} \sqrt{\frac{\sin \theta}{\varepsilon}-1}}, \\
& k_{z 2}= \pm k_{0} \sqrt{\left(\varepsilon-\sin ^{2} \theta\right)-\varepsilon^{\prime} \sqrt{\frac{\sin \theta}{\varepsilon}-1}} .
\end{aligned}
$$

We are interested in the wave propagating downward into the medium, so we consider the negative solutions. The Helmholtz equation 5.13 with $k_{z 1}$ is then

$$
\left(\begin{array}{ccc}
-\epsilon^{\prime} \sqrt{\frac{\sin ^{2} \theta}{\epsilon}-1} & \epsilon^{\prime} & 0 \\
-\epsilon^{\prime} & \sin ^{2} \theta-\epsilon^{\prime} \sqrt{\frac{\sin ^{2} \theta}{\epsilon}-1} & -\sin \theta \sqrt{\epsilon-\sin ^{2} \theta+\epsilon^{\prime} \sqrt{\frac{\sin ^{2} \theta}{\epsilon}-1}} \\
0 & -\sin \theta \sqrt{\epsilon-\sin ^{2} \theta+\epsilon^{\prime} \sqrt{\frac{\sin ^{2} \theta}{\epsilon}-1}} & \epsilon-\sin ^{2} \theta
\end{array}\right)\left(\begin{array}{l}
\hat{E}_{x} \\
\hat{E}_{y} \\
\hat{E}_{z}
\end{array}\right)=0,
$$

which, along with equation 5.20, provides us with equations for the components of the $\mathbf{E}$ and $\mathbf{H}$ fields in terms of $\hat{E}_{x}$ :

$$
\begin{gathered}
\hat{E}_{y}^{\left(t_{1}\right)}=\sqrt{\frac{\sin ^{2} \theta}{\epsilon}-1} \hat{E}_{x}^{\left(t_{1}\right)}, \\
\hat{E}_{z}^{\left(t_{1}\right)}=-\frac{\sin \theta \sqrt{\epsilon-\sin ^{2} \theta+\epsilon^{\prime} \sqrt{\frac{\sin ^{2} \theta}{\epsilon}-1}}}{\epsilon \sqrt{\frac{\sin ^{2} \theta}{\epsilon}-1}} \hat{E}_{x}^{\left(t_{1}\right)}, \\
H_{x}^{\left(t_{1}\right)}=-\frac{\sqrt{\epsilon-\sin ^{2} \theta+\epsilon^{\prime} \sqrt{\frac{\sin ^{2} \theta}{\epsilon}-1}}}{\sqrt{\frac{\sin ^{2} \theta}{\epsilon}-1}} \hat{E}_{x}^{\left(t_{1}\right),} \\
H_{y}^{\left(t_{1}\right)}=-\sqrt{\epsilon-\sin ^{2} \theta+\epsilon^{\prime} \sqrt{\frac{\sin ^{2} \theta}{\epsilon}-1}} \hat{E}_{x}^{\left(t_{1}\right)}, \\
H_{z}^{\left(t_{1}\right)}=-\sin \theta \hat{E}_{x}^{\left(t_{1}\right)} .
\end{gathered}
$$

We now return to the Helmholtz equation 5.13 and consider the second wave 
vector, $k_{z 2}$, to obtain

$$
\left(\begin{array}{ccc}
\epsilon^{\prime} \sqrt{\frac{\sin ^{2} \theta}{\epsilon}-1} & \epsilon^{\prime} & 0 \\
-\epsilon^{\prime} & \sin ^{2} \theta+\epsilon^{\prime} \sqrt{\frac{\sin ^{2} \theta}{\epsilon}-1} & -\sin \theta \sqrt{\epsilon-\sin ^{2} \theta-\epsilon^{\prime} \sqrt{\frac{\sin ^{2} \theta}{\epsilon}-1}} \\
0 & -\sin \theta \sqrt{\epsilon-\sin ^{2} \theta-\epsilon^{\prime} \sqrt{\frac{\sin ^{2} \theta}{\epsilon}-1}} & \epsilon-\sin ^{2} \theta
\end{array}\right)\left(\begin{array}{l}
\hat{E}_{x} \\
\hat{E}_{y} \\
\hat{E}_{z}
\end{array}\right)=0 .
$$

As before, we use this matrix along with equation 5.20 to obtain the $\mathbf{E}$ and $\mathbf{H}$ field components, but this time in terms of $\hat{E}_{y}$ :

$$
\begin{gathered}
\hat{E}_{x}^{(t 2)}=-\frac{1}{\sqrt{\frac{\sin ^{2} \theta}{\epsilon}-1}} \hat{E}_{y}^{(t 2)}, \\
\hat{E}_{z}^{(t 2)}=\frac{\sin \theta}{\epsilon-\sin ^{2} \theta} \sqrt{\epsilon-\sin ^{2} \theta-\epsilon^{\prime} \sqrt{\frac{\sin ^{2} \theta}{\epsilon}-1}} \hat{E}_{y}^{(t 2)}, \\
\hat{H}_{x}^{(t 2)}=\frac{\epsilon}{\epsilon-\sin ^{2} \theta} \sqrt{\epsilon-\sin ^{2} \theta-\epsilon^{\prime} \sqrt{\frac{\sin ^{2} \theta}{\epsilon}}-1} \hat{E}_{y}^{(t 2)}, \\
\hat{H}_{y}^{(t 2)}=\frac{\sqrt{\epsilon-\sin ^{2} \theta-\epsilon^{\prime} \sqrt{\frac{\sin ^{2} \theta}{\epsilon}-1}} \hat{E}_{y}^{(t 2)},}{\sqrt{\frac{\sin ^{2} \theta}{\epsilon}-1}} \\
\hat{H}_{z}^{(t 2)}=\frac{\sin ^{2} \theta}{\sqrt{\frac{\sin ^{2} \theta}{\epsilon}-1}} \hat{E}_{y}^{(t 2)} .
\end{gathered}
$$

At this point, the equations can be greatly simplified by assuming normal incidence (i.e. $\theta=0$ ). Equations 5.29 and 5.31 then become: 


$$
\begin{aligned}
& k_{z 1}=-k_{0} \sqrt{\epsilon+i \epsilon^{\prime}} \\
& \hat{E}_{y}^{\left(t_{1}\right)}=i \hat{E}_{x}^{\left(t_{1}\right)} \\
& \hat{E}_{z}^{\left(t_{1}\right)}=0 \\
& \hat{H}_{x}^{\left(t_{1}\right)}=i \sqrt{\epsilon+i \epsilon^{\prime}} \hat{E}_{x}^{\left(t_{1}\right)} \\
& \hat{H}_{y}^{\left(t_{1}\right)}=-\sqrt{\epsilon+i \epsilon^{\prime}} \hat{E}_{x}^{\left(t_{1}\right)} \\
& \hat{H}_{z}^{\left(t_{1}\right)}=0 \\
& k_{z 2}=-k_{0} \sqrt{\epsilon-i \epsilon^{\prime}} \\
& \hat{E}_{x}^{\left(t_{2}\right)}=i \hat{E}_{y}^{\left(t_{1}\right)} \\
& \hat{E}_{z}^{\left(t_{2}\right)}=0 \\
& \hat{H}_{x}^{\left(t_{2}\right)}=\sqrt{\epsilon-i \epsilon^{\prime}} \hat{E}_{y}^{\left(t_{1}\right)} \\
& \hat{H}_{y}^{\left(t_{2}\right)}=-i \sqrt{\epsilon-i \epsilon^{\prime}} \hat{E}_{y}^{\left(t_{1}\right)} \\
& \hat{H}_{z}^{\left(t_{2}\right)}=0
\end{aligned}
$$

These fields have no z components, and in each case $\hat{H}_{x}$ and $\hat{H}_{y}$ differ only by a factor of $-i$, indicating that within the magnetic medium, the natural modes are right circular polarization (RCP) and left circular polarization (LCP), respectively. Given this, the refractive indices are necessarily

$$
\begin{aligned}
& n_{1}=\sqrt{\epsilon+i \epsilon^{\prime}}, \\
& n_{2}=\sqrt{\epsilon-i \epsilon^{\prime}} .
\end{aligned}
$$

The reflectivity can be calculated from[50]

$$
R=\left(\frac{n_{1}-n_{2}}{n_{1}+n_{2}}\right) .
$$

Thus, from equations 5.34 and 5.35, one can calculate the reflectivity of the RCP and LCP components of the incident light, and the polarization of the reflected beam is therefore given by superposition of the resulting components. 


\section{Magnetic Microscopy}

Kerr microscopy is far from being the only available technique for magnetic imaging. Indeed, magnetic fields were imaged before they had ever been understood. It was the tendency of iron filings to align in predictable patterns around a bar magnet that first allowed Faraday to conceptualize magnetic field lines, eventually to be mathematically quantified by Maxwell[51]. Magnetic imaging has come a long way since those days. Some other modern methods of magnetic microscopy include the Bitter method, electron microscopy (SEM and TEM), and magnetic force microscopy (MFM). MFM is of particular interest as a tool for comparison and verification of results obtained with the Kerr system.

\subsection{The Bitter Method}

Bitter's method derives from a simple premise - that magnetic particles will align in the direction of the magnetic field. In this method, a colloidal mixture of ferromagnetic or ferrimagnetic particles is applied to the surface of a sample. This solution, once known as a Bitter solution, is now commonly referred to as a ferrofluid. In a ferrofluid, magnetic particles are coated with a surfactant to prevent agglomeration before being suspended in a carrier fluid. The result is a paramagnetic fluid which tends to accumulate along the domain walls of the sample. This is known as "decorating" the sample. The particles may then be imaged using any of several techniques. With high-resolution imaging (i.e., electron microscopy or scanning tunneling microscopy), the resolution of the Bitter method is limited only by the size of the particles, which may be as small as $10 \mathrm{~nm}[44]$.

\subsection{Magnetic Force Microscopy}

Magnetic force microscopy (MFM), pioneered by Martin and Wickramasinghe in 1987[52], is the most widely used technique for magnetic imaging. As the name 
implies, MFM is an extension of atomic force microscopy (AFM). A typical AFM reveals the topography of a sample in non-contact or tapping mode by monitoring the response of a driven cantilever as it nears the surface, as described in section 4.2. At this close range, atomic forces affect the oscillation.

In an MFM, the cantilever is coated with a ferromagnetic film, such as $\mathrm{CoCr}[53]$, CoPt[54], or Ni[54]. The tip is then magnetized by application of an external field, thereby leading to a magnetic force between the tip and sample. The first line of topography is scanned as normal, and the weak magnetic force has little effect on overall picture. At this point, MFM deviates from AFM. The tip is lifted above the sample by a user-specified distance, the lift height, and the topography is retraced. In this way, the contributions from the atomic forces are effectively negated, and the only deflection in the tip can be attributed to the magnetic force, which, though relatively weak, persists over a longer distance than the others[44].

Using a commercial AFM (Park Scientific Instruments) with CoCr MFM tips (MikroMasch NSC18), we have successfully obtained MFM data. Figures 6.1 and 6.2 show sectors on a floppy data disk and the magnetic structure of a MnGe film, respectively.

In the final stages of this project, MFM will be crucial for sample analysis and verification of data.

\subsection{Electron Microscopy Techniques for Magnetic Imaging}

Electron microscopy is a valuable tool in modern scientific research. As was discussed in section 3.6, resolution in optical microscopy is limited by diffraction according to Abbe's equation:

$$
d=\frac{\lambda}{2(n \sin \alpha)},
$$

where $d$ is the resolution limit, $\lambda$ is the wavelength of propagation, and $n \sin \alpha$ is the numerical aperture. This limit applies in electron microscopy as well; however, 

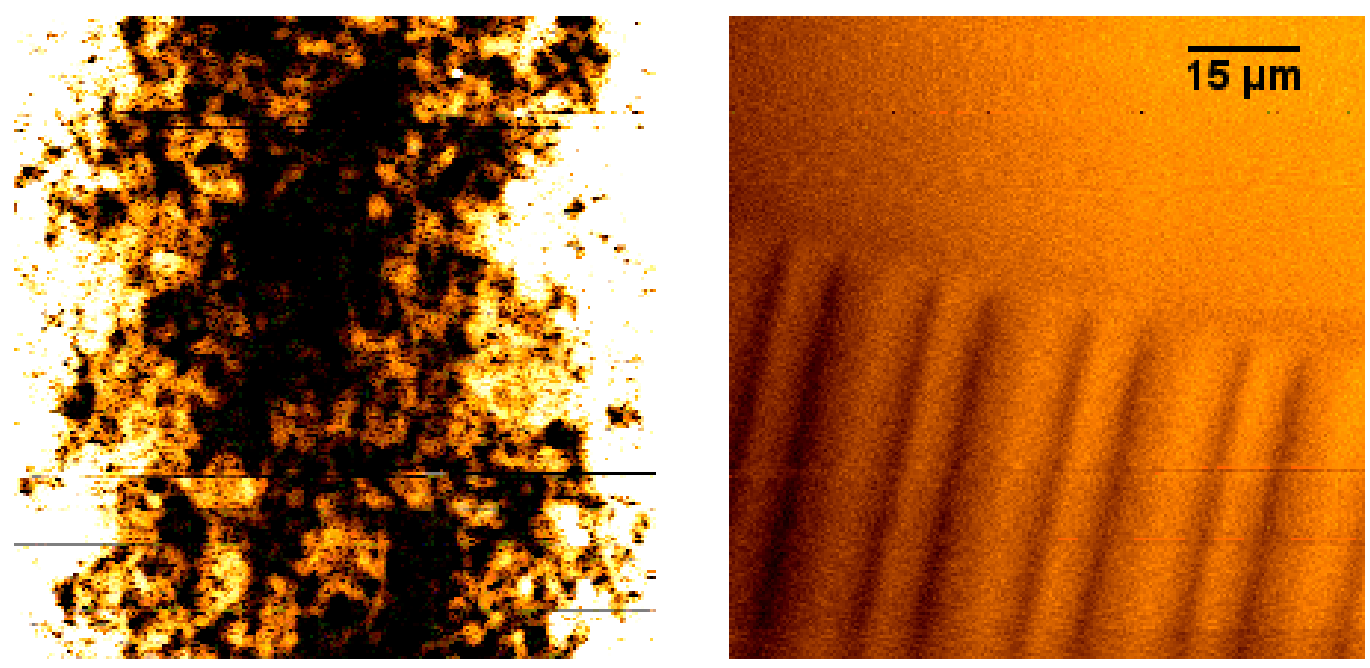

Figure 6.1: Simultaneous AFM topography (left) and MFM phase signals (right) reveal sectors of floppy data disk.
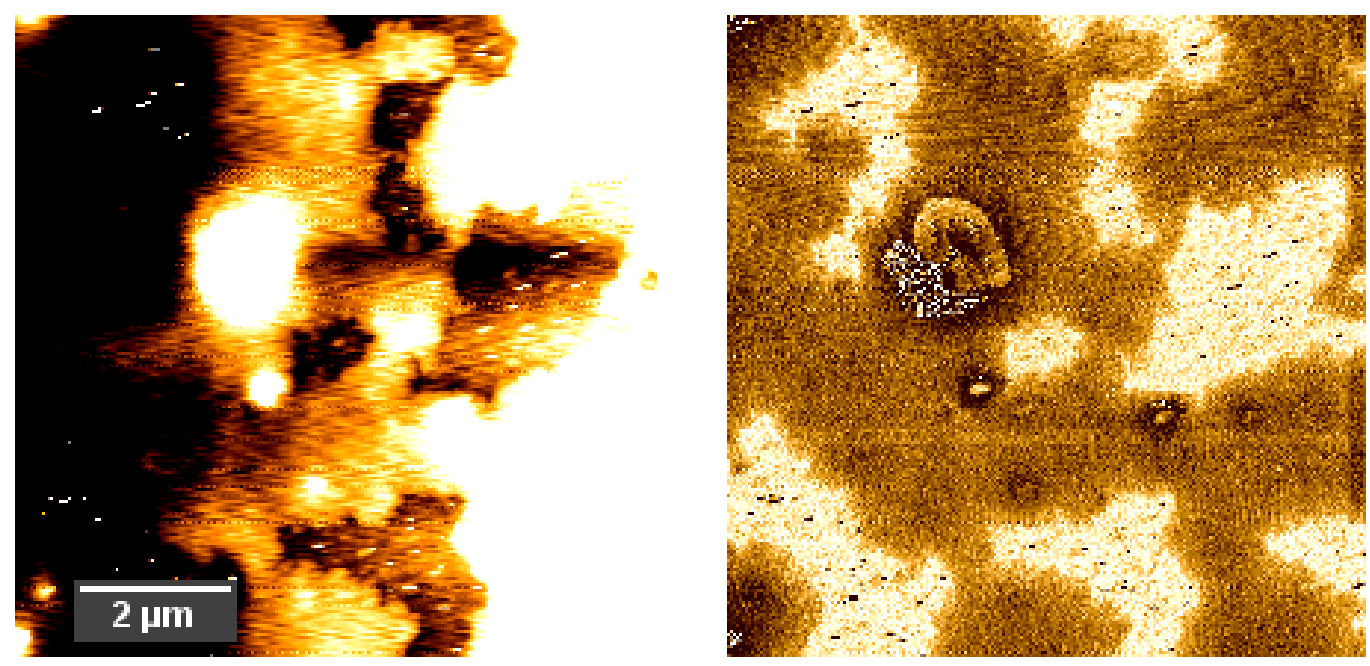

Figure 6.2: Simultaneous AFM topography (left) and MFM phase (right) signals of MnGe film.

the wavelength of electron propagation is considerably shorter than that of visible light. Whereas visible light has wavelength 400-700 nm, the wavelength of electron propagation, referred to as the de Broglie wavelength in honor of Louis de Broglie, is given by $[55]$ :

$$
\lambda=\frac{h}{m_{e} v},
$$


where $h$ is Planck's constant, $m_{e}$ is the electron mass, and $v$ is the electron's velocity. Using the standard equation for kinetic energy, which will give us a fairly accurate result despite ignoring significant relativistic effects, eliminates the unknown velocity from the equation. We then have

$$
\lambda=\frac{h}{\sqrt{2 E_{k} m_{e}}},
$$

and the resolution limit due to diffraction is therefore

$$
d=\frac{h}{2(n \sin \alpha) \sqrt{2 E_{k} m_{e}}} .
$$

In typical operating conditions (accelerating voltage of 1-100 keV), equation 6.3 yields a de Broglie wavelength on the order of $10 \mathrm{pm}$, about four orders of magnitude lower than the wavelength of visible light. If we assume the half angle $\alpha$ of the aperture to be roughly .01 radians [55], we find that the resolution limit is on the order of $1 \mathrm{~nm}$. Aberrations and distortions in lenses and apertures somewhat reduce the resolution from this theoretical optimum. To date, the highest resolution obtained by a scanning electron microscope (SEM) was 80 pm[56]. Transmission electron microscopy (TEM) typically acquires even better resolution than SEM-using an accelerating voltage of $300 \mathrm{kV}$ and half angle of $28.9 \mathrm{mrad}$ with advanced aberration correction techniques, $50 \mathrm{pm}$ resolution has been obtained using a TEM[57].

With such improved resolution over optical microscopy, it is unsurprising that electron microscopes have become so prevalent in so many fields of scientific research. Magnetic microscopy is no exception. There are many methods by which magnetic structure can be investigated with electron microscopy. The most common of these are Lorentz microscopy and scanning electron microscopy with polarization analysis (SEMPA). 
In Lorentz microscopy, a thin magnetic sample is investigated with a TEM. As the electron beam passes through the sample, the interaction due to the Lorentz force, given by

$$
\mathbf{F}=q[\mathbf{E}+(\mathbf{v} \times \mathbf{B})]
$$

results in a measurable deflection, which serves as the contrast mechanism. This method has achieved lateral resolution of $10 \mathrm{~nm}[58]$. SEMPA relies on a Mott detector to measure the spin polarization of secondary electrons in an SEM[59]. This provides a direct measurement of the magnitude and direction of the sample magnetization. SEMPA is an incredibly sensitive technique with demonstrated resolution as low as $10 \mathrm{~nm}[60]$.

Electron microscopy is clearly a powerful tool for investigation of magnetic samples, however, it is not without its disadvantages. Lorentz microscopy in particular is limited to very thin samples. While SEMPA is a more versatile technique, it has all of the disadvantages that come with electron microscopy, namely the necessity for a vacuum, charging of ungrounded samples, and cost of maintenance. A more relevant disadvantage to all of these techniques is the interaction of the magnetic field in the vicinity of the sample with the imaging probe. Because it is generally desirable to image the magnetization of the sample itself, the interference of the stray field may be an unwanted complication[61].

\subsection{Kerr Microscopy}

Kerr microscopy, as the name implies, harnesses the magneto-optic Kerr effect to image magnetic domains. Before the invention of electron microscopes and atomic force microscopes, Kerr microscopy was the only alternative to the Bitter method.

Traditional Kerr setups employ two crossed polarizers in an otherwise standard optical microscope. The first polarizer ensures the purity of the polarization of the illumination light. The second polarizer, referred to as the analyzer, is crossed with 
the first for maximum extinction such that, in the absence of polarization effects at the sample, the detector will receive no light. Therefore, the rotation of the polarization plane due to the Kerr effect will allow some light to pass through the analyzer, forming an image of the magnetic structure. A conceptual diagram of such an arrangement is shown in figure 6.3.

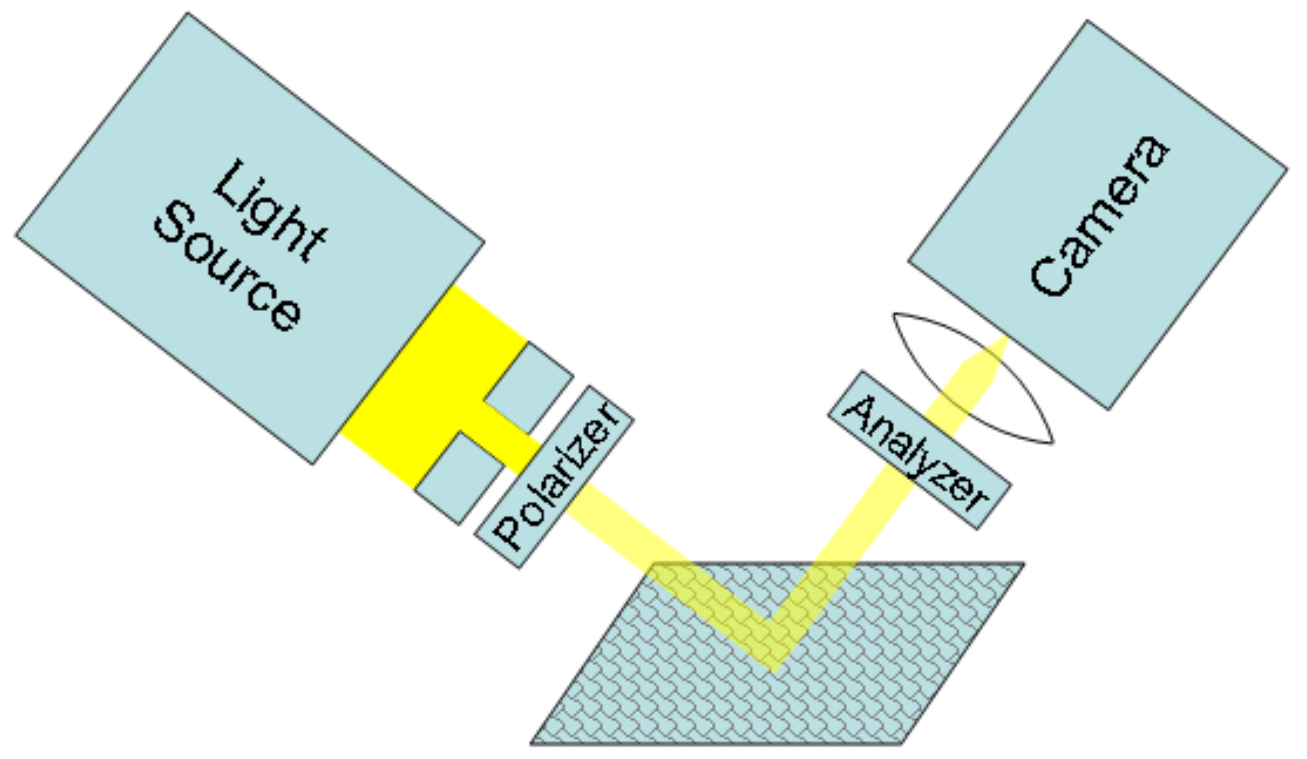

Figure 6.3: Traditional configuration of Kerr microscope.

After some time was spent investigating this configuration, the determination was made that the Kerr rotation angle was too small, and the Kerr signal therefore too weak, to be detected above the noise in our system. Consequently, we decided to use an alternative configuration, based on the principles that allow magneto-optical (MO) drives to read the magnetic state of data bits.

MO Drives employ a differential detection scheme using a polarizing beam splitter (PBS), which separates light into its s and p components. The incident light reflects from the MO data disk into the PBS, and the s and $\mathrm{p}$ components are collected by separate photodiodes. The initial polarization is set such that the intensity of the s 
and p polarized components are equal in the absence of a Kerr rotation. Therefore a rotation will contribute a slight increase to the signal in one detector and a slight decrease to the other. The difference between the two signals, which would be zero in the absence of the Kerr effect, therefore reveals only information related to the Kerr rotation $[5]$.

We have built the same detection scheme into our microscope. Two APDs of the same model employed previously (APD110A) were mounted to the outputs of a cube-mounted polarizing beam splitter (Thorlabs CM1-PBS251), while the initial polarization was set by rotation of a half-wave plate (Thorlabs WPH05M-514). Figure 6.4 shows these modifications to the optical microscope.

The differential detection requirement posed another challenge. The FPGA code was not configured to receive input from two detectors, so that section had to be rewritten. Moreover, it was desirable to monitor both signals as well as the difference signal simultaneously, which required modification to the scanning portion of the Windows code. This program now monitors three signals simultaneously, and the user may choose between any of the available options, which include topography, phase, $\operatorname{APD}(\mathrm{s}), \operatorname{APD}(\mathrm{p})$, and APD (ms-np), where $\mathrm{m}$ and $\mathrm{n}$ are user-defined variables to allow for fine adjustment of the relative intensities of the $\mathrm{s}$ and $\mathrm{p}$ signals before subtraction. The updated GUI is shown in figure 6.5

Initial investigations have been performed on magneto-optical disks due to the relatively strong Kerr rotation of the rare earth-transition metal (RE-TM) films they employ. In figure 6.6, magnetic contrast is clearly seen as the black and white variation along the tracks. Figure 6.7 is a three-dimensional representation of the difference signal, showcasing these features. 


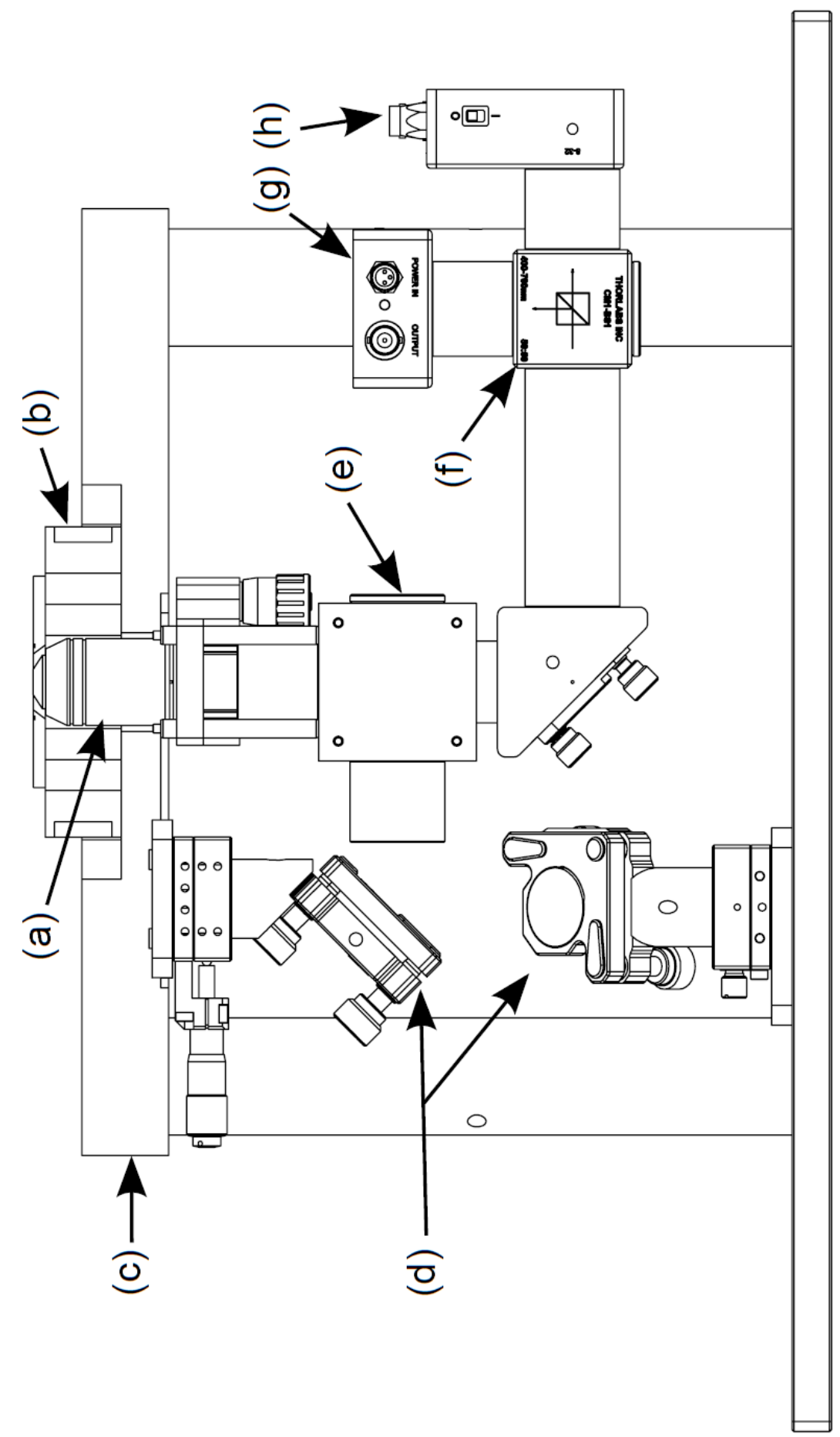

Figure 6.4: SolidWorks Diagram of Kerr Microscope a) Objective lens b) Scan stage c) Base plate d) Periscope assembly e) Beam splitter f) Polarizing beam splitter g) APD for s signal h) APD for p signal. 


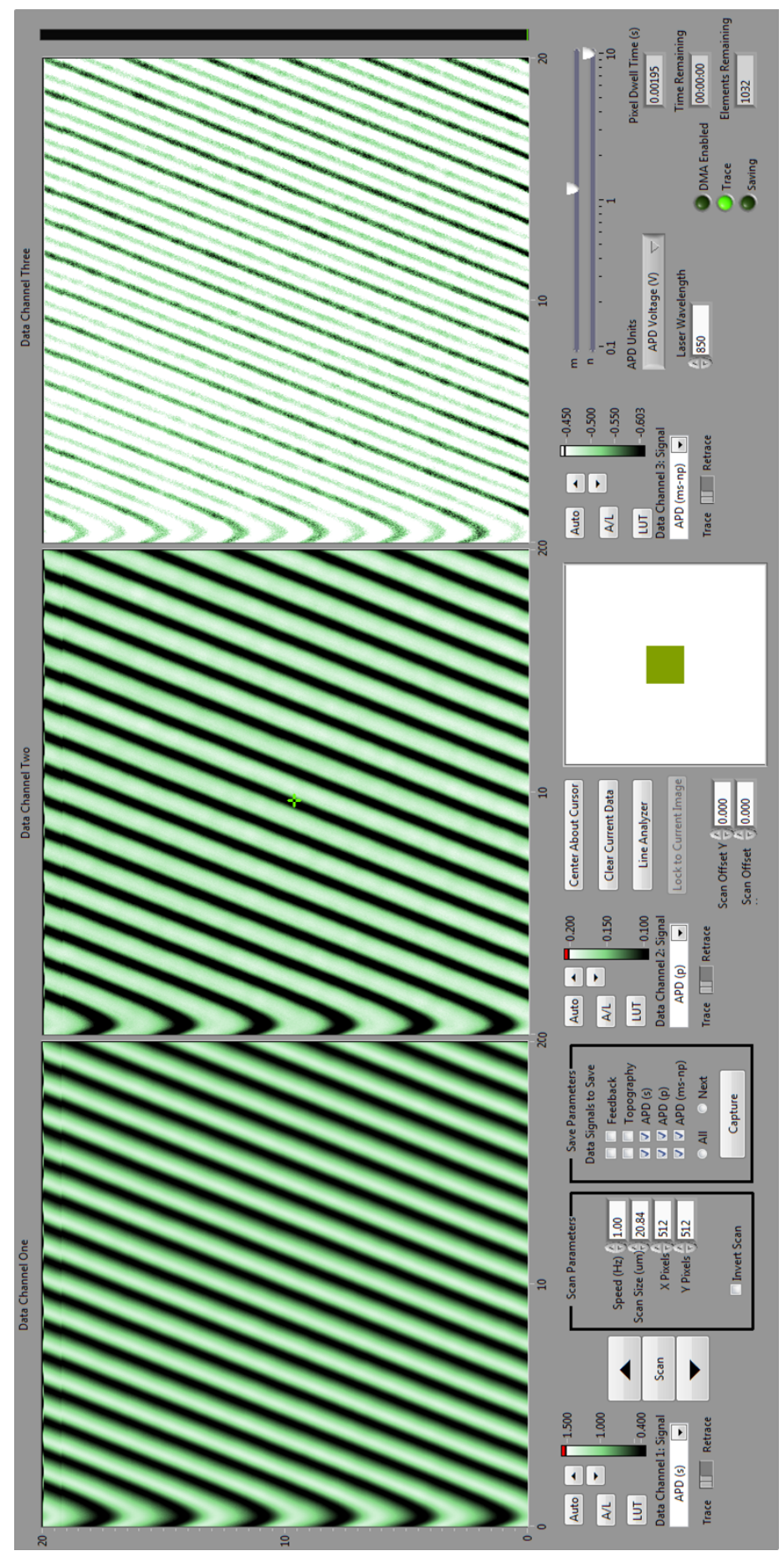

Figure 6.5: Updated GUI for scan program provides monitoring of three channels to display s, p, and difference signals simultaneously. The mirror pattern on the left of each image is an artifact of the closed loop scanner. 


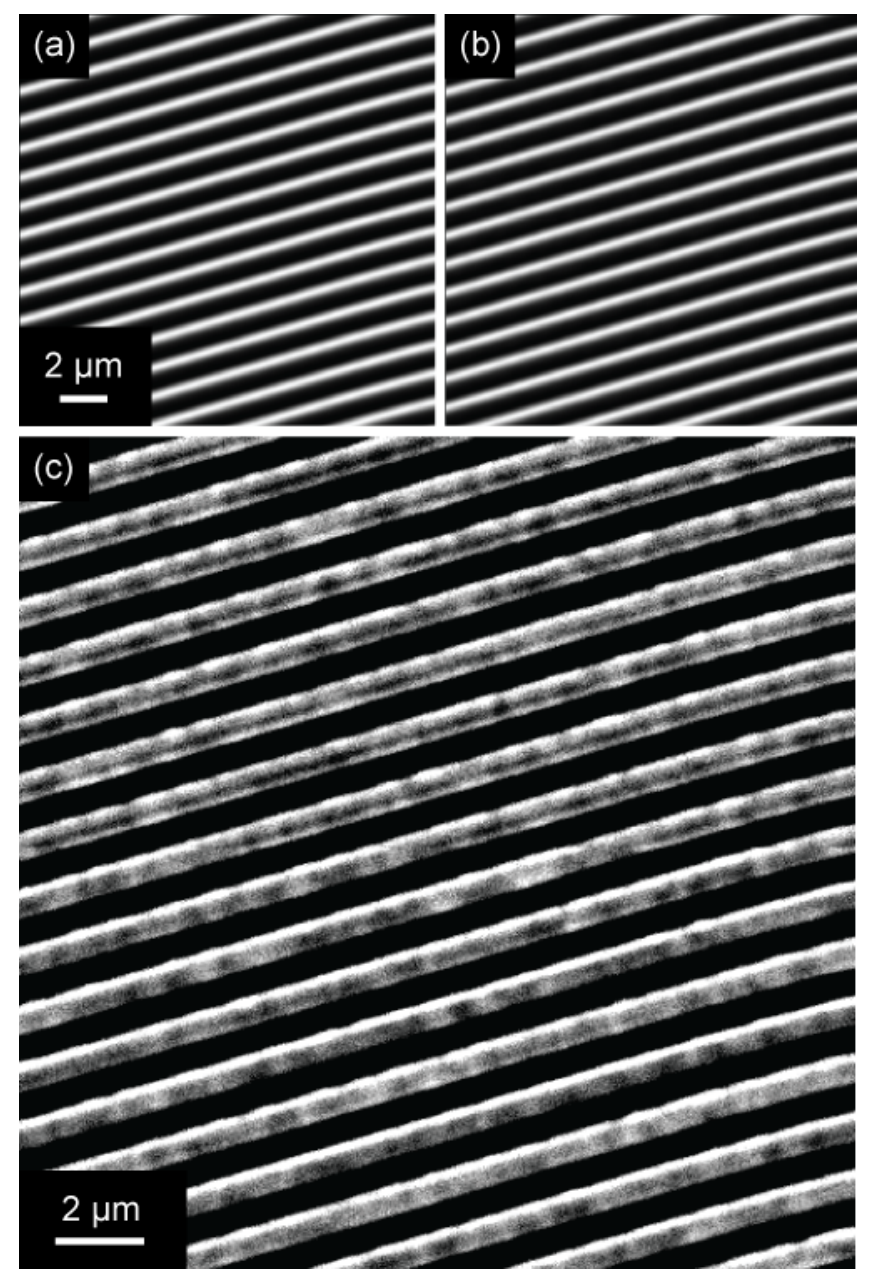

Figure 6.6: Kerr image of magneto-optic disk. a) s component of reflected light b) p component c) Difference signal revealing magnetic contrast along data tracks. 


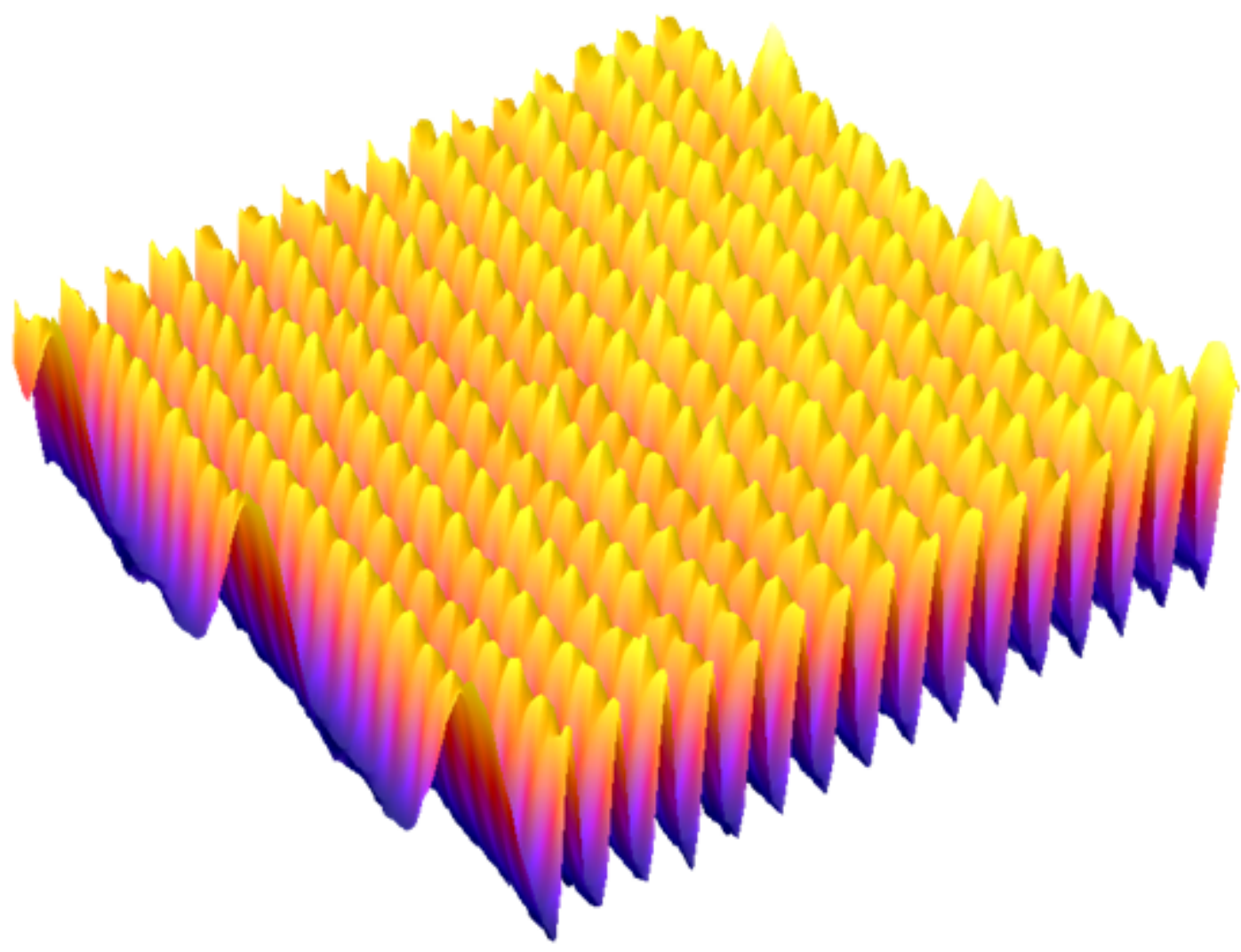

Figure 6.7: Three dimensional Kerr image reveals data bits on a magneto-optic disk. In this representation, the peaks corresponding to the bit structure are extremely apparent. 


\section{Writing Magnetic Domains}

If near-field Kerr is ever to be used as a technology for data storage, then it is not sufficient that the instrument be able to image magnetic information. It must also be capable of altering the magnetic state of a sample at the same resolution - writing as well as reading. This requires a heating mechanism to bring the sample above the Curie temperature, an external magnetic field, and precise control of the sample position.

\subsection{Magnetic Domains}

In section 5.4, we demonstrated that the off-diagonal components of the dielectric tensor lead to a change in the refractive index. This affects the polarization of the reflected and transmitted light and is therefore responsible for the Kerr and Faraday effects. The off-diagonal components of the dielectric tensor are determined by the magnetization of the medium, and we may therefore conclude that the magnitude of the Kerr rotation is directly dependent on the sample magnetization.

The magnetization over an entire sample, however is not generally uniform, and Kerr microscopy would hardly be of any interest if that were the case. Instead, mag-

netic media are composed of many small regions of uniform magnetization, known as magnetic domains. Within these regions, all of the atoms possess magnetic moments which point in the same direction[44].

The orientation of magnetic domains is motivated by energy minimization. The magnetic free energy within a crystal is given by[62]

$$
E=E_{e x}+E_{k}+E_{\lambda}+E_{D}+E_{H},
$$

where the terms on the right correspond to the exchange, magnetocrystalline aniso- 
tropy, magnetoelastic, magneto-static, and Zeeman energies, respectively, of the crystal. The magneto-static energy, $E_{D}$, is of particular interest. This is the energy due to the magnetic field created by interaction of the magnetic domains with one another, and minimization of this term occurs when the domains are aligned such that magnetization makes closed loops, with parallel magnetization occurring between adjacent domains. The result is that ferromagnetic materials appear with statistically random domains and no net field[62].

Paramagnetic materials, by contrast, have no domain structure. The magnetic moments of the atoms in these materials will align in the presence of a magnetic field, but the dipoles do not interact with each other. Susceptibility, the degree of magnetization due to the presence of a magnetic field, is therefore higher in paramagnets than ferromagnets. Their orientation in the absence of an external field appears completely random due to thermal motion. As ferromagnetic materials are heated, the thermal energy approaches the interaction energy between the dipoles. The temperature at which these energies are equal is known as the Curie temperature, and a ferromagnet heated above its Curie temperature will therefore become paramagnetic.[44]

The result is that reorientation of ferromagnetic domains should be possible with localized heating to bring the sample above its Curie temperature. If an external field is applied in this paramagnetic state, the domain should refreeze in the direction of the applied field.

\subsection{Design of Electromagnet}

The requirements for reorientation of magnetic domains, then, are heat and an external magnetic field. In a magneto-optical disk drive, a system which shares many similarities with our microscope, the field is supplied by a magnetic recording head, while the heat comes from increasing the intensity of the illumination laser, typically to around $10 \mathrm{~mW}[5]$ - a value which our laser systems are easily capable of outputting. 
Due to the dynamic nature of the Kerr effect, however, it was not sufficient to use a simple field as would be provided by a recording head or solenoid. We required control of the field intensity in all three axes, and therefore endeavored to create an electromagnetic unit in which the total field vector could be controlled in three dimensions. The SolidWorks design and a photograph of the functional magnet are shown in figures 7.1 and 7.2 , respectively.

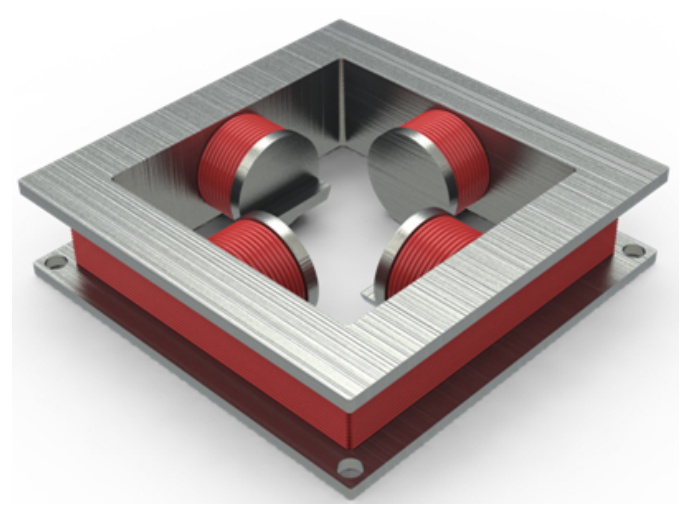

Figure 7.1: SolidWorks rendering of vectorizable electromagnet.

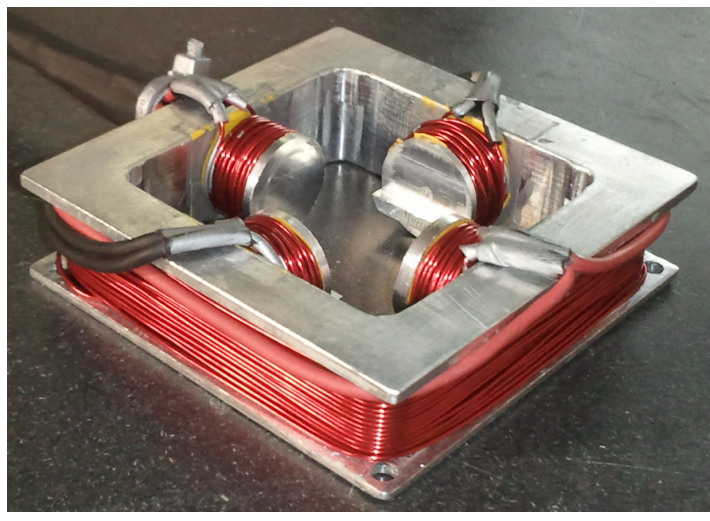

Figure 7.2: Photograph of functional electromagnet.

As with most of the design aspects of the system, the magnet was drafted in SolidWorks. The major complication was in obtaining adequate field strength within the narrow physical range afforded by the existing microscope design. The unit needed to mount to the piezo scan stage, allowing for an overall footprint of just (67 $\mathrm{mm})^{2}$, while not interfering with the objective lens or scan head and also holding the sample. This goal likely would have proved unattainable without the assistance in visualization provided by CAD. With the aim of maximization of field strength and homogeneity, the unit was designed as a variation of the "Maltese cross" [63] design, in which a field is generated in the center of two sets of two concentric solenoids. These take the form of four cylindrical arms which provide the $\mathrm{x}$ and $\mathrm{y}$ fields. The arms are mounted to the inside of a square solenoid, which provides the z field, while 
a shelf on the arms suspends the sample in the proximity of the objective lens.

The electromagnet was machined from aluminum to minimize the remnant field of the unit when not in use, and custom LabVIEW software (figure 7.3) controls the strength and direction of the generated magnetic field.

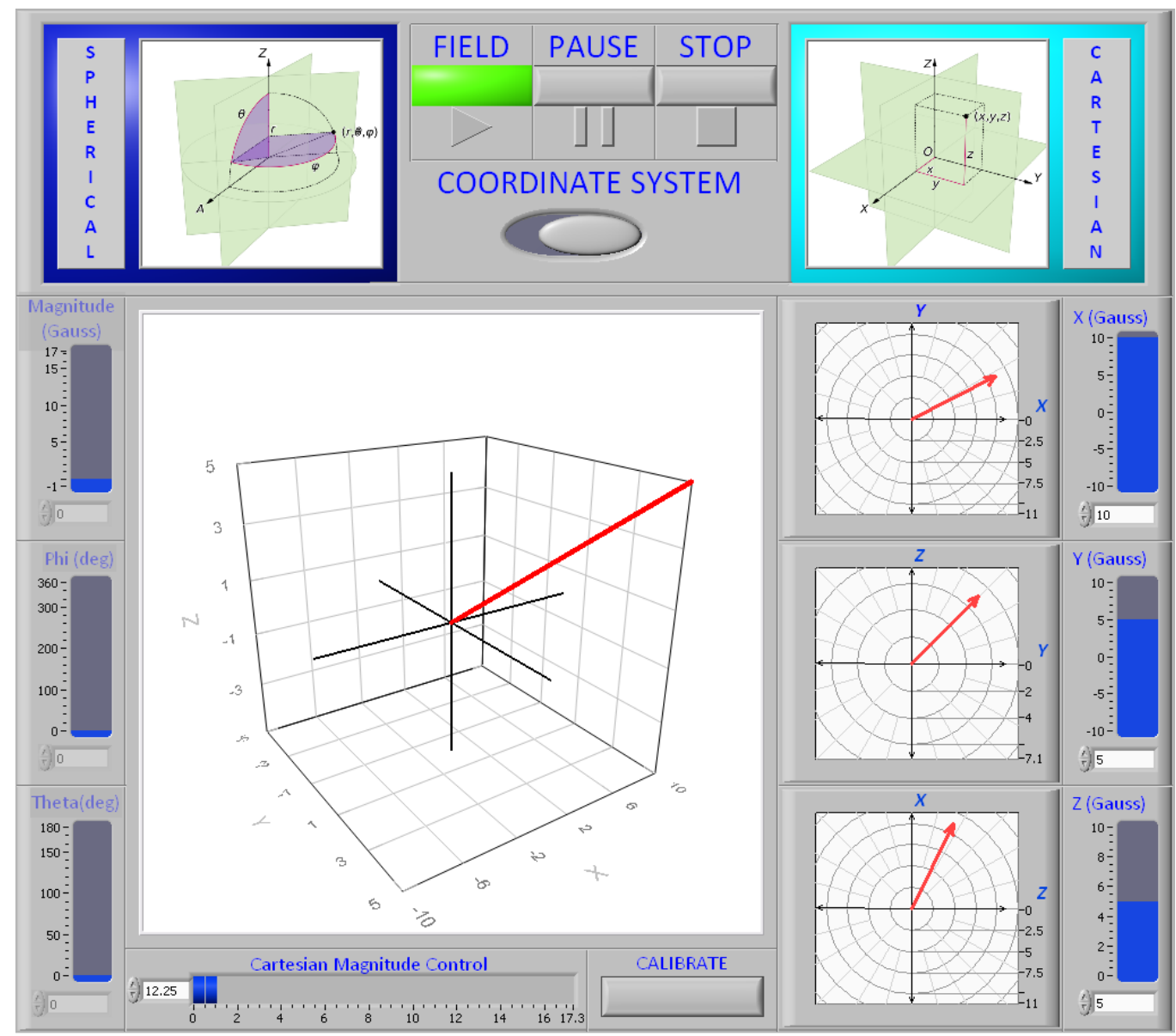

Figure 7.3: GUI for electromagnet control software, written in LabVIEW.

\subsection{Magnetic Field of a Circular Solenoid}

The magnetic field due to a circular solenoid (figure 7.4) can be derived from a straightforward application of the Biot-Savart law[64]:

$$
\mathbf{B}(\mathbf{r})=\frac{\mu_{0}}{4 \pi} \int \frac{\mathbf{I} \times r}{r^{2}} d \mathbf{l}^{\prime}
$$


In this equation, $\mu_{0}$ is the permeability of free space, $\mathbf{r}$ is the vector from the source to the point, and $d \mathbf{l}^{\prime}$ is the length element in the direction of the current. From figure 7.4 we see that this becomes

$$
B=\frac{\mu_{0} I}{4 \pi} \int \frac{d l^{\prime}}{r^{2}} \cos \theta=\frac{\mu_{0} I}{4 \pi} \frac{2 \pi R}{r^{2}} \cos \theta
$$

Substituting

$$
\cos \theta=\frac{R}{\sqrt{R^{2}+z^{2}}}
$$

and multiplying by $\mathrm{N}$ loops, we obtain

$$
\mathbf{B}=\frac{\mu_{0} N I R^{2}}{2\left(R^{2}+z^{2}\right)^{3 / 2}} \hat{\mathbf{z}},
$$

which is the final form for the magnetic field of a circular solenoid.

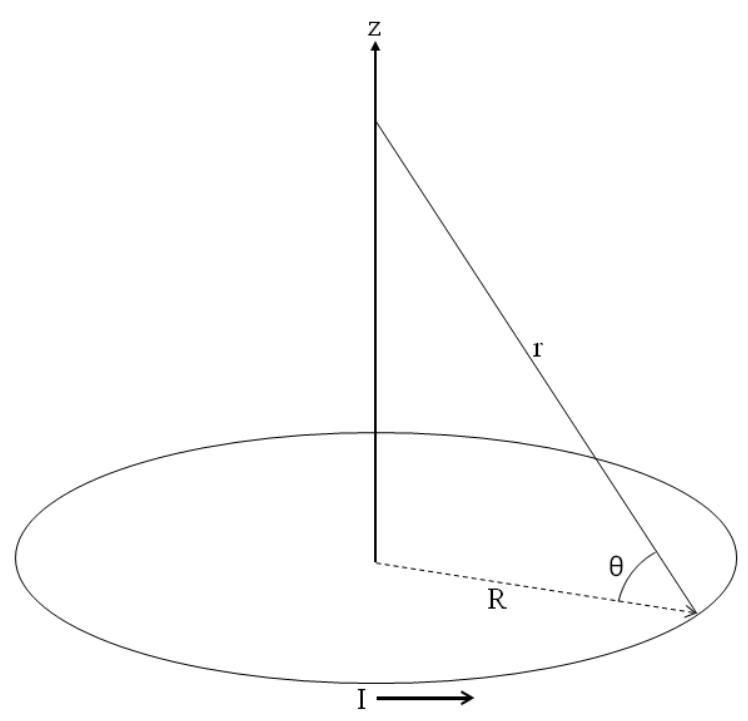

Figure 7.4: Magnetic field of a circular loop. 


\subsection{Magnetic Field of a Square Solenoid}

The treatment of a square solenoid is less straightforward. We again begin with BiotSavart law, equation 7.2, and calculate the magnetic field at an arbitrary point due to a current flowing through a wire, as shown in figure 7.5:

$$
\mathbf{B}(\mathbf{r})=\frac{\mu_{0}}{4 \pi} I \int \frac{d \mathbf{l}^{\prime} \times \hat{\mathbf{r}}}{r^{2}}
$$

From figure 7.5,

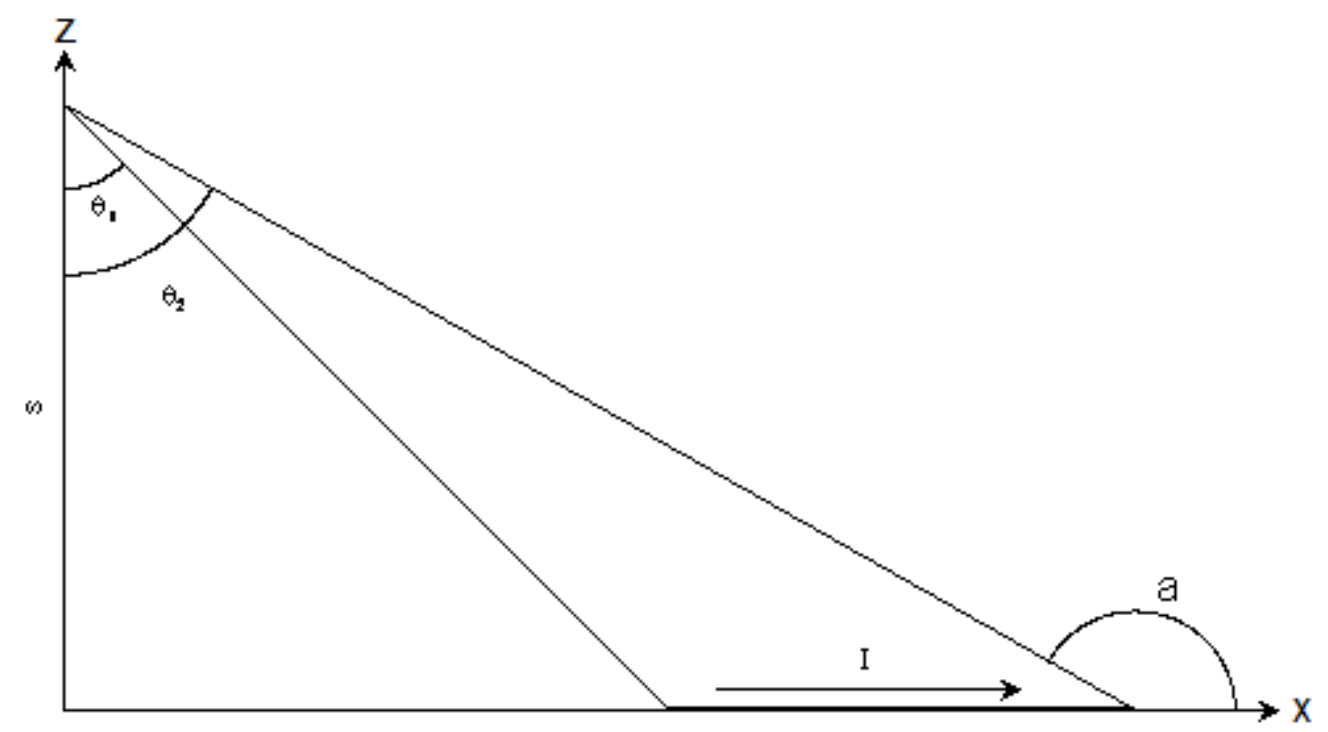

Figure 7.5: Magnetic field of a wire segment.

$$
\left|d \mathbf{l}^{\prime} \times \hat{\mathbf{r}}\right|=d l^{\prime} \sin \alpha=d l^{\prime} \cos \theta
$$

We can also see that $l^{\prime}=s \tan \theta$. Therefore

$$
d l^{\prime}=\frac{s d \theta}{\cos ^{2} \theta}
$$


Further, we have that $s=r \cos \theta$, so

$$
r=\frac{s}{\cos \theta}
$$

Substituting equations 7.7 through 7.9 into equation 7.6 yields

$$
B=\frac{\mu_{0} I}{4 \pi} \int_{\theta_{1}}^{\theta_{2}} \frac{\cos \theta}{s} d \theta=\frac{\mu_{0} I}{4 \pi s}\left(\sin \theta_{2}-\sin \theta_{1}\right) .
$$

Equation 7.10 is the general form which represents the field at any point due to a current-carrying wire. A square loop of wire, figure 7.6, may be approximated as four such wire segments.

We use equation 7.10 to consider the field due to one half of each wire segment. From the Pythagorean theorem, we clearly see that

$$
s=\sqrt{z^{2}+\left(\frac{w}{2}\right)^{2}}
$$

and $\mathrm{r}$ is therefore

$$
r=\sqrt{s^{2}+\left(\frac{w}{2}\right)^{2}}=\sqrt{z^{2}+\frac{w^{2}}{2}} .
$$

We take $\theta_{1}$ as 0 and see that

$$
\sin \theta_{2}=\frac{\frac{w}{2}}{r}
$$

We then proceed to plug equations 7.11-7.13 back into equation 7.10, and find that

$$
B=\frac{\mu_{0} I}{4 \pi s} \sin \theta_{2}=\frac{\mu_{0} I}{4 \pi} \frac{w}{2 r s}=\frac{\mu_{0} I}{4 \pi} \frac{w}{2 \sqrt{z^{2}+\frac{w^{2}}{2}} \sqrt{z^{2}+\left(\frac{w}{2}\right)^{2}}} .
$$

Due to symmetry, all non z components will cancel between the four sides of the 


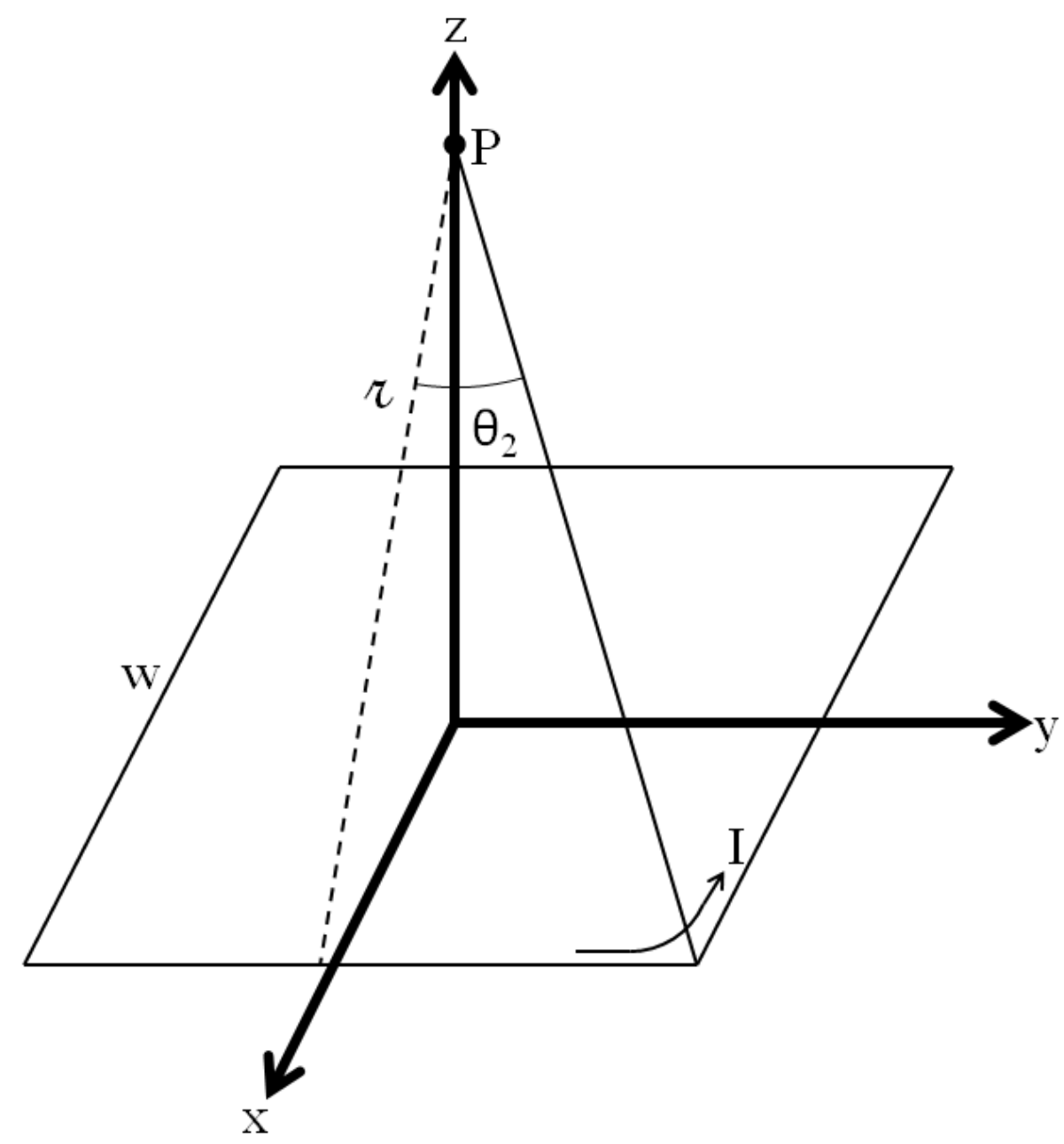

Figure 7.6: Magnetic field of a square loop.

square. We therefore multiply by

$$
\sin \phi=\frac{\left(\frac{w}{2}\right)}{\sqrt{z^{2}+\left(\frac{w}{2}\right)^{2}}}
$$

to obtain

$$
\mathbf{B}=\frac{\mu_{0} I}{16 \pi} \frac{w^{2}}{\left(z^{2}+\frac{w^{2}}{4}\right) \sqrt{z^{2}+\frac{w^{2}}{2}}} \hat{\mathbf{z}} .
$$

Equation 7.16 gives one eighth of the total field for a square loop (half of one side of 
the square). For $\mathrm{N}$ loops, the total field is then given by

$$
\mathbf{B}=\frac{\mu_{0} N I}{2 \pi} \frac{w^{2}}{\left(z^{2}+\frac{w^{2}}{4}\right) \sqrt{z^{2}+\frac{w^{2}}{2}}} \hat{\mathbf{z}} .
$$

Equations 7.5 and 7.17, then, are the relevant formulae to the calculations of our magnetic fields.

\subsection{Magnetolithography}

The electromagnet was found to produce magnetic fields the strength of which was roughly in agreement with theory. Plugging in actual values for the unknowns in equations 7.5 and 7.17 yields predicted values of 4.9, 4.5, and 27.5 gauss for the $\mathrm{x}$, y, and z axes, respectively, while measurement with a DC magnetometer (AlphaLab, Inc.) yielded 2.69, 2.28, and 16.24 gauss[65].

With the electromagnet in place and the scanning optical microscope functional, the basic requirements for reorientation of magnetic domains were accounted for. Using a similar method to that employed in section 3.5 for photobleaching via successive scanning, we attempted to write a $(20 \mu \mathrm{m})^{2}$ square on a magneto-optical disk by scanning only that region. By applying an external magnetic field and increasing the laser intensity to approximately $30 \mathrm{~mW}$, we were successful in modifying the sample in the region of interest, as shown in figure 7.7 .

However, comparison of the s and p channels revealed little difference, indicating that the modification was not a magnetic effect. Rather, it seems likely that the high power of the laser partially melted the lacquer coating on the disk, resulting in a less reflective surface. Further attempts at writing magnetic domains with this method proved unsuccessful. This may have been due to differential heating owed to imperfect laser alignment, inadequate field strength, lack of precision in targeting the region of interest, or some as yet unknown factor. Regardless, it was determined that 


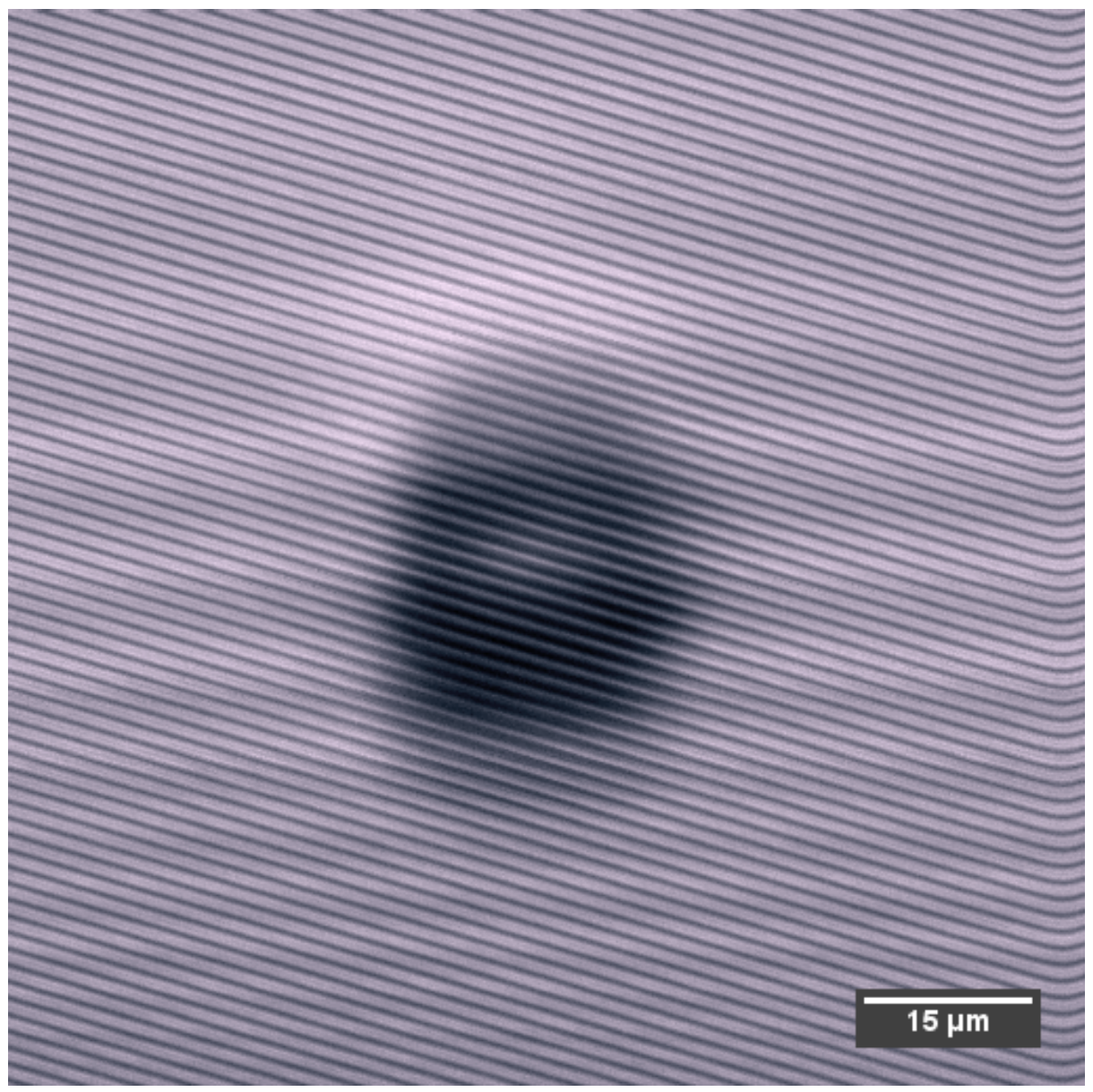

Figure 7.7: Preliminary attempt at writing a magnetic pattern in the shape of a square.

writing in the far field on a magneto-optical disk would have little carryover into near field domain writing and the endeavor was therefore abandoned as a low priority.

The lithography technique employed so far has been adequate for testing purposes. By scanning a small area with the lithographic light before scanning a larger area, an area of contrast becomes visible. However, this allows only for square patterns, which are not terribly interesting or useful for any other purpose. Therefore, a lithography program, shown in figure 7.8 , is in development.

The program has been fully integrated with the main ANSOM Project code in LabVIEW and allows the user to import a monochrome bitmap or draw simple shapes, 


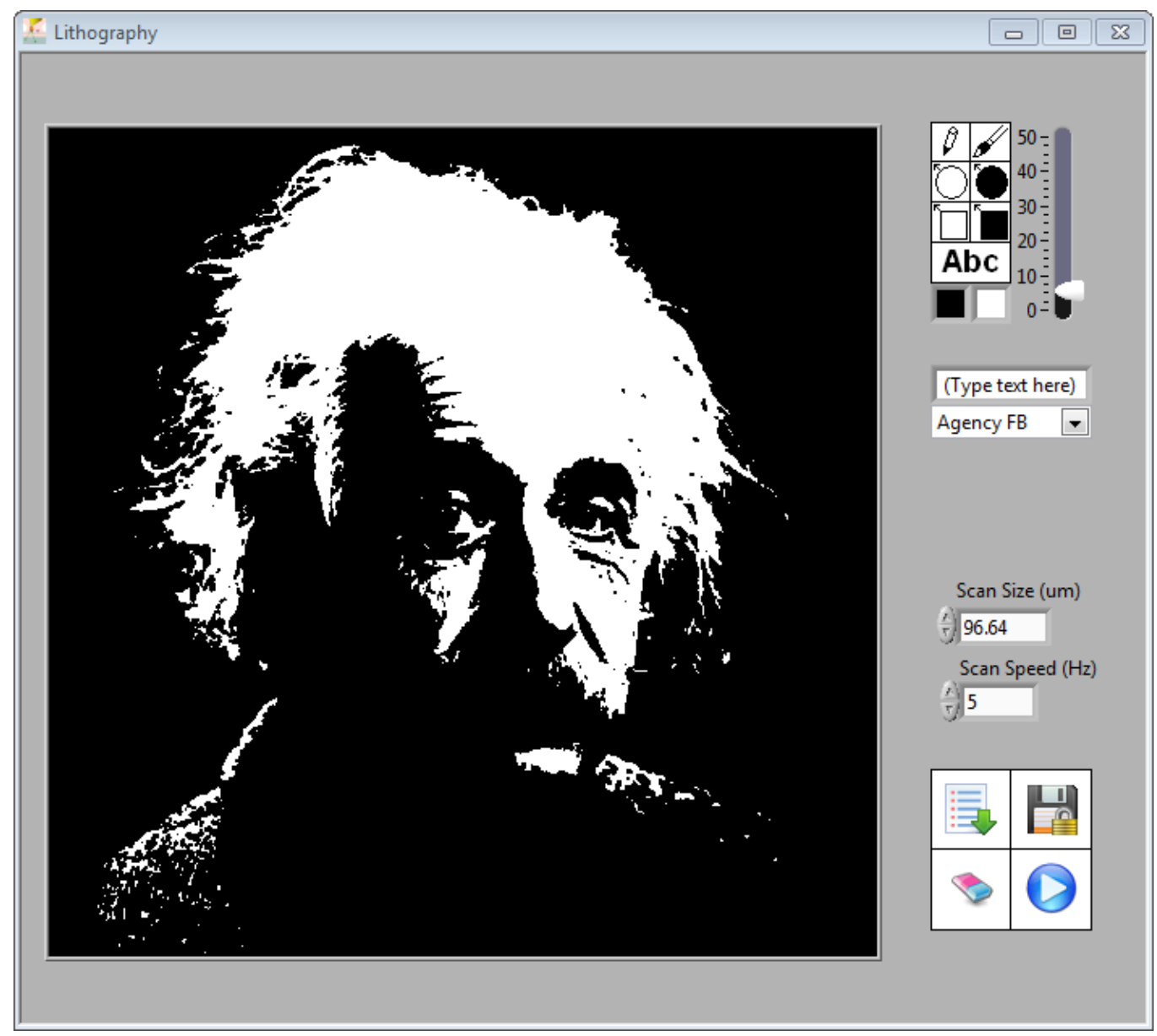

Figure 7.8: GUI for lithography program.

including dots, lines, circles, squares, and text. These patterns can also be saved for later use. The graphical user interface (GUI) for this program is fully functional, but the FPGA code which would direct the scanner to write the pattern remains incomplete. 


\section{Fiber Microscopy}

With the far-field Kerr system imaging successfully, the next step was improvement of the resolution. As discussed in section 3.6, the resolution in optical microscopy is fundamentally limited by diffraction to roughly half the wavelength of the incident light. This limit, however, clearly applies only to propagating light. The evanescent field within one wavelength of the source (the so called near field) is non-propagating and thus not subject to diffraction. The near field can be used as an imaging probe by placing a very small aperture in the path of the beam, such that the opening is too narrow for diffraction to occur. This is traditionally accomplished with the use of tapered optical fibers.

\subsection{Optical and Atomic Force Microscopy with Fiber Probes}

The fabrication of fiber probes is a complicated process. Once the cladding of the fiber has been removed, both ends are mounted in a quartz micropipette puller (Sutter P-2000). As the core of the fiber is heated with a 20W Class IV CO2 laser, the two ends are pulled in opposite directions. The simultaneous application of heat and tension stretches the fiber until the ends separate, resulting in an extremely sharp tip on each of the separated segments. These are then sputter coated (Plasma Sciences Inc. CrC-100 Sputtering System) with a thin ( 100 nm) layer of gold before being loaded into a focused ion beam (FIB) system (Micrion 2500).

Once in the FIB, the geometry of the probe can be tailored to more specific applications using the milling capabilities of the ion beam[66]. Ultimately, this project may require a very specific and sophisticated probe design. The necessary theory and computational work to make that determination are complicated and the research is ongoing[67]. For the present, however, it is sufficient to cut the apex of the tip to reveal an aperture through which light can escape. Such a cut is shown in figure 8.1. 


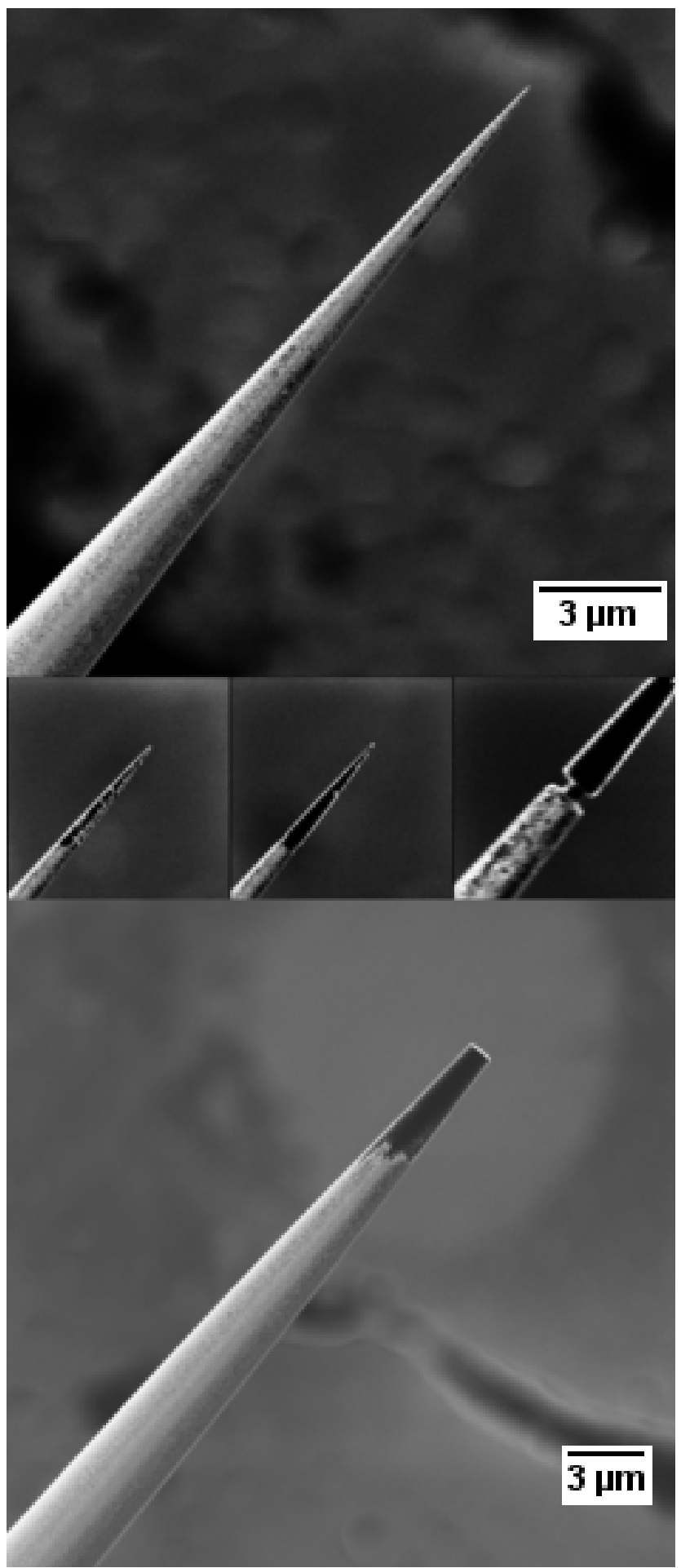

Figure 8.1: Pulled optical fiber in various stages of FIB milling process. 
Implementation of such a probe required some modification, as neither the AFM nor the optical microscope was originally configured to image via a fiber probe, and each presents its own set of problems. AFM becomes challenging due to the length requirement of the probe. In order to propagate light into the fiber, it must be long enough to extend from the tuning fork to the top of scan head and into a fiber splice. The absolute minimum functional length is therefore about $15 \mathrm{~cm}$, and in reality, should be closer to $20 \mathrm{~cm}$ or greater to provide adequate flexibility. Compared with a typical probe of less than a centimeter, this represents a significant increase in length and therefore mass, resulting in severe damping of the tuning fork to the point where the feedback response is inadequate for SFM imaging, as shown in figure 8.2.

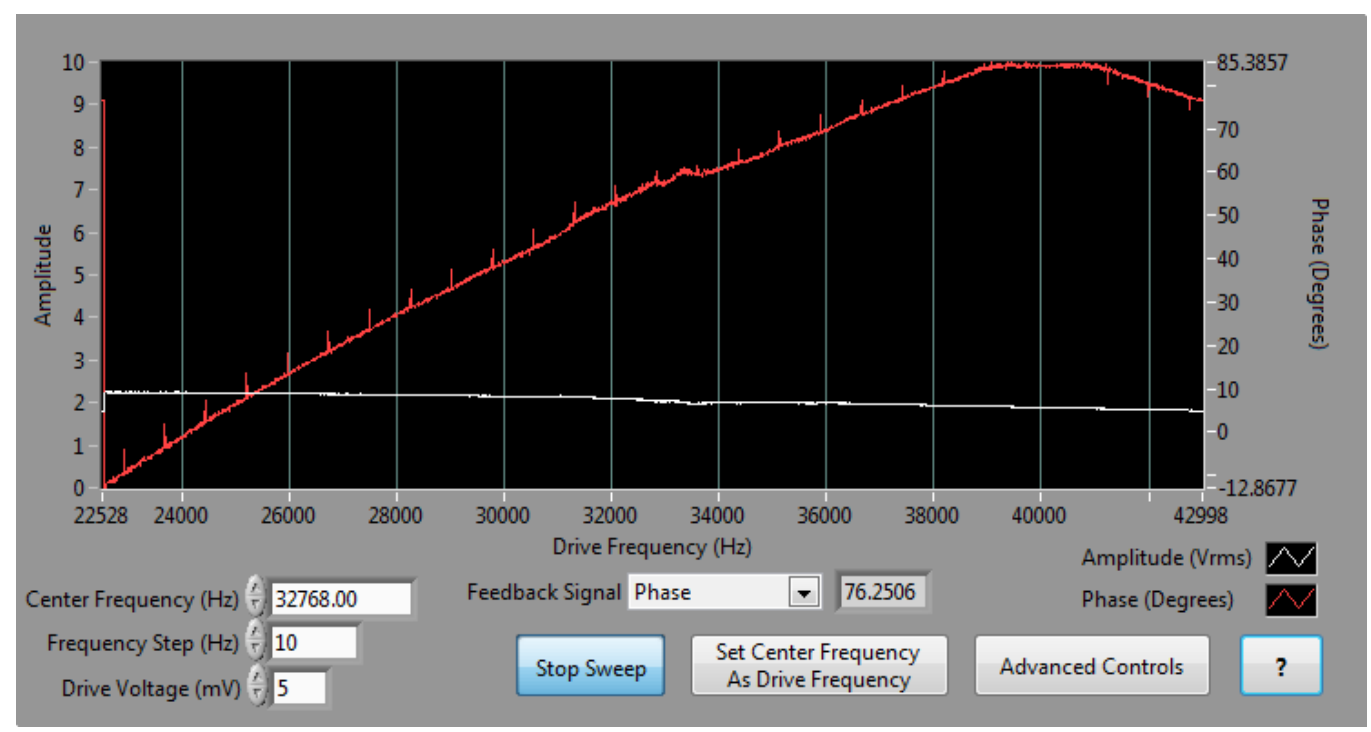

Figure 8.2: AFM sweep with long fiber attached to tuning fork.

Fortunately, this problem can be alleviated by affixing the fiber directly to the tuning fork circuit board with a small dab of adhesive. This serves to isolate the bulk of the fiber probe from the tuning fork, resulting in a vastly improved feedback response (figure 8.3), as well as protecting the probe and tuning fork from damage due to accidental tension. 


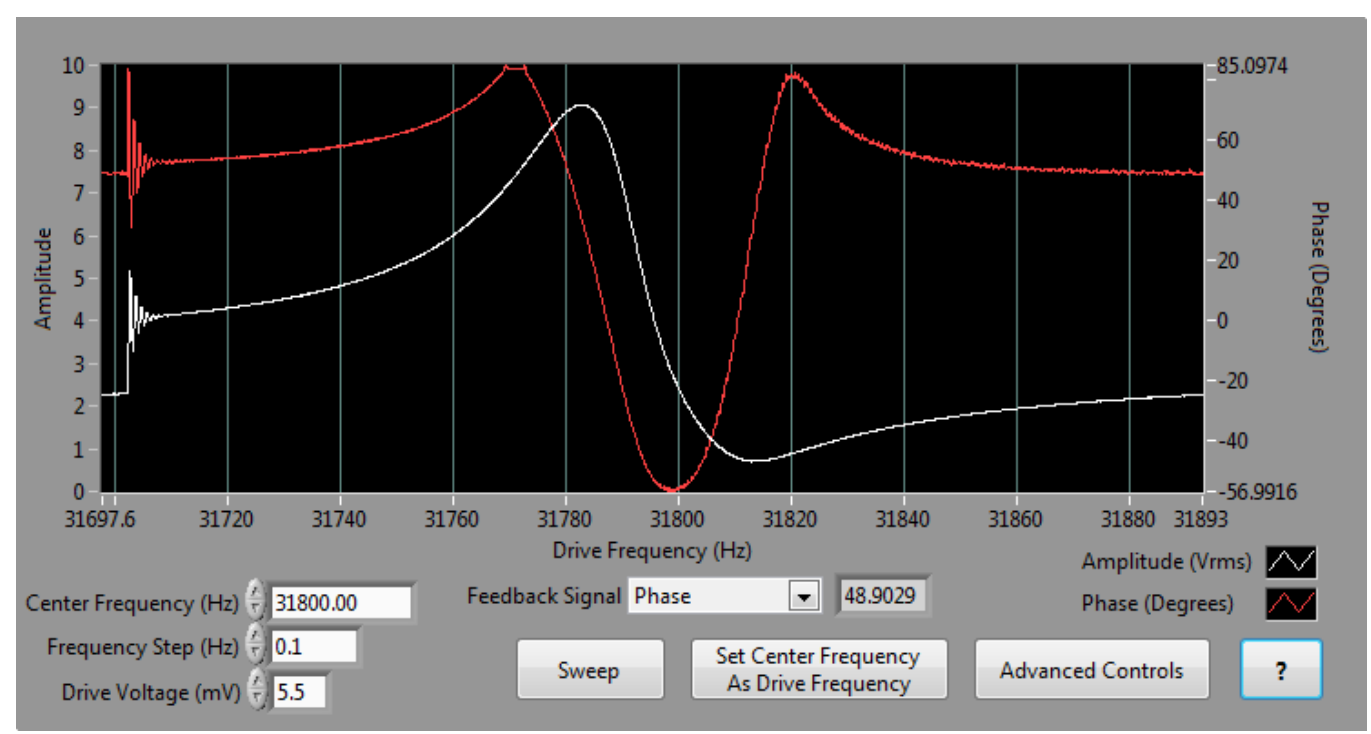

Figure 8.3: AFM sweep with fiber glued to tuning fork circuit board.

The second modification required was to the optical pathway. The illumination laser was redirected from the periscope assembly to a three-axis fiber launch (Thorlabs MBT612), in which an objective lens (Carl Zeiss 4188853, Neofluar 6,3/0,20) focuses the laser light into the cleaved fiber with roughly $90 \%$ throughput. The other end of the fiber was then coupled to the imaging probe using a fiber to fiber splice (Thorlabs TS128), which in turn was mounted to the backplane assembly. Figure 8.4 shows the illuminated fiber probe as it approaches the sample.

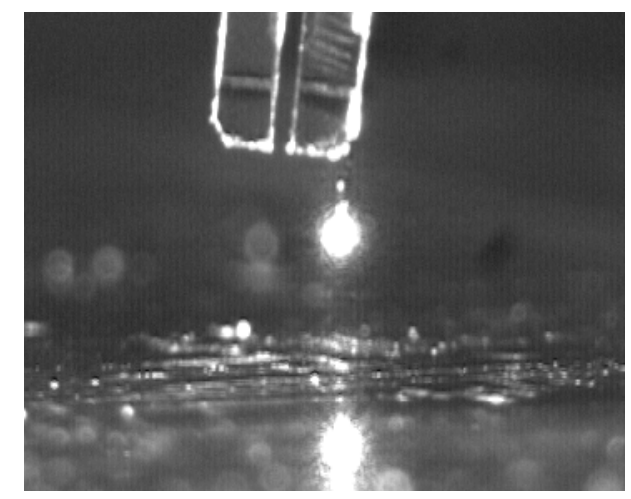

Figure 8.4: Illuminated fiber probe approaching sample surface. 


\subsection{Probe Fabrication}

The research we are undertaking in probe optimization is ongoing. We have not yet finalized our design, as we are still investigating the complicated interplay between various parameters. It is probable that the final design will utilize a bowtie pattern milled out of a triangular prism shape, as seen in figures 8.5 and 8.6, which show a SolidWorks model to be imported into finite difference time domain (FDTD) electromagnetic field modeling software (Remcom).

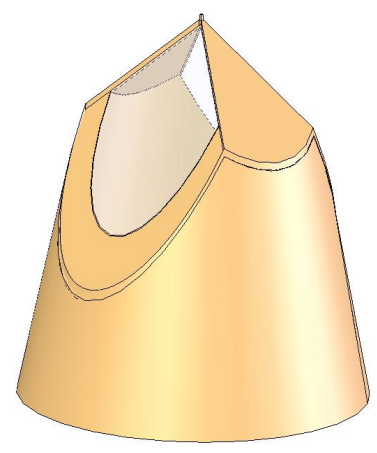

Figure 8.5: CAD model of fiber probe for FDTD analysis.

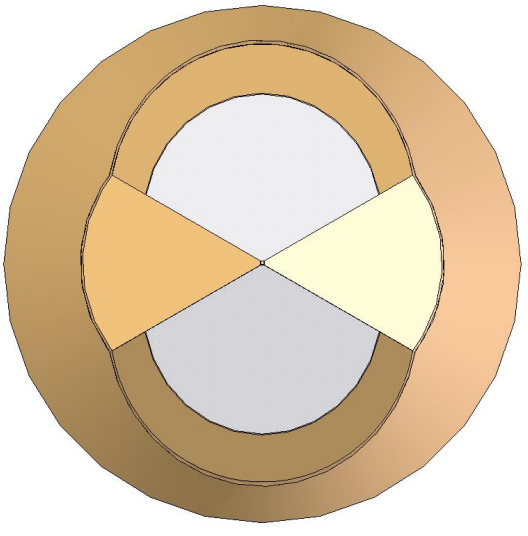

Figure 8.6: Top down view of CAD fiber probe.

We have fabricated some preliminary probes as a proof of concept. These probes were prepared with the fiber puller and sputter coater as detailed in the preceding section before being loaded into the FIB (FEI Strata 237 Dual Beam). Figure 8.7 is an SEM image showing the pulled fiber coated in gold. After cutting the prism shape, as seen in figure 8.8, the probe was removed from the FIB chamber, coated a second time (roughly $200 \mathrm{~nm}$ thick), and then reloaded into the chamber, resulting in the probe seen in figure 8.9.

The native milling capabilities of the FIB are well suited to circles, lines, and even simple polygons, but are entirely inadequate when attempting to fabricate more complex patterns, such as the desired bowtie shape. Fortunately, the FIB software 
is capable of reading bitmap files. We therefore imported the pattern seen in figure 8.10 and milled through the entire coating. Figures 8.11 shows the results.

The final step in the process was a simple cleanup of the top surface of the gold coating by milling from the side, leaving us with the probe seen in figures 8.12 and 8.13.

\subsection{Fiber Microscopy Results}

For initial testing, the detector was left beneath the sample for transmission mode imaging of a thin film of gold sputter-coated on a glass microscope slide. The scan revealed elevated features in the topographic signal which corresponded to dark spots in the optical signal, as seen in figure 8.14. These features may indicate areas of poor adhesion between the glass and the gold.

\subsection{Future Work}

A fairly straightforward modification of the microscope would be the replacement of the detector with the polarizing beam splitter (PBS) detection assembly. In this way, the instrument would become sensitive to the changes induced in the polarization by transmission through a magnetic sample (the Faraday Effect). While this would be an interesting course of study, the ultimate goal of the project is better served by pressing forward. The next step is therefore to collect the reflected light for eventual analysis by Kerr microscopy. A fraction of the light emitted through the fiber is reflected

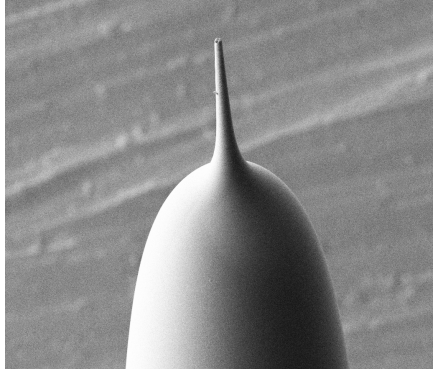

Figure 8.7: Gold coated fiber. $\mathrm{FOV}=80 \mu \mathrm{m}$.

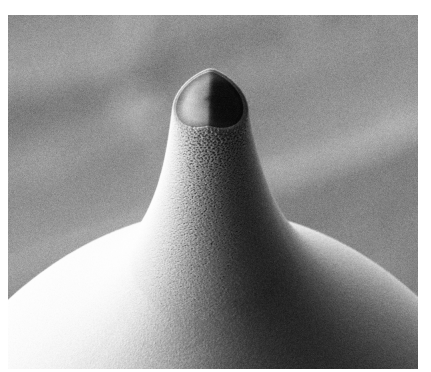

Figure 8.8: First cut on fiber probe.

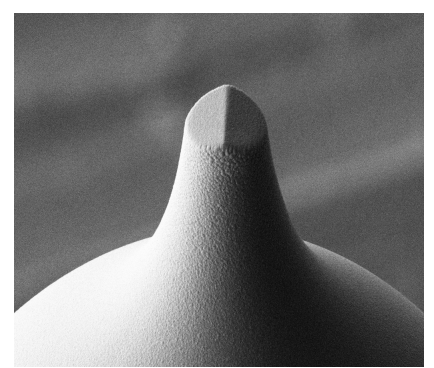

Figure 8.9: Fiber probe with second gold coating. 


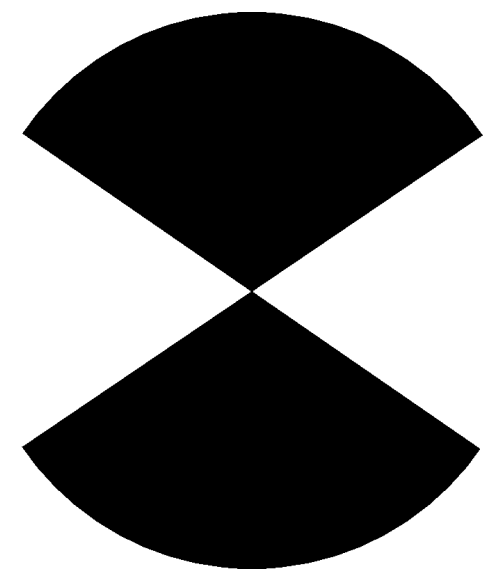

Figure 8.10: Bitmap used for bowtie cut.

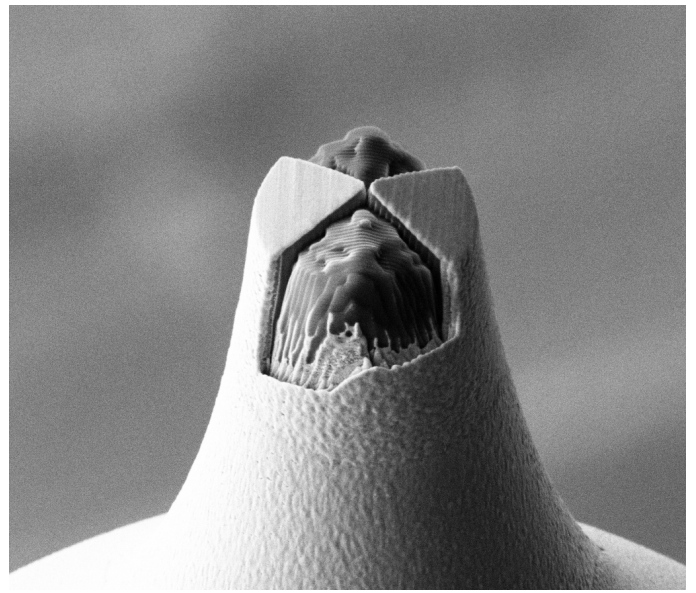

Figure 8.12: Bowtie probe.

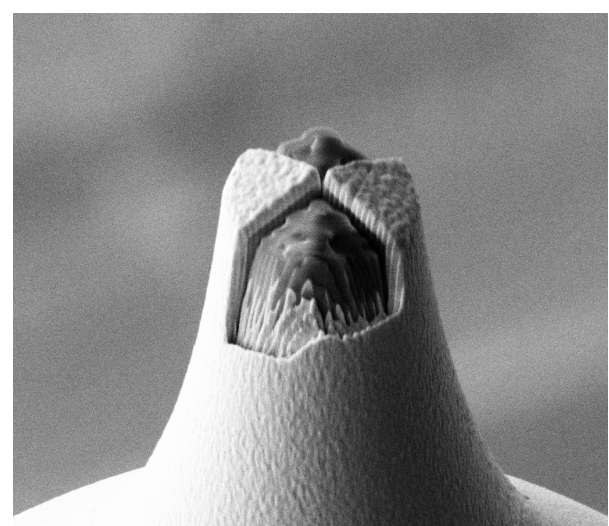

Figure 8.11: Fiber after bowtie cut.

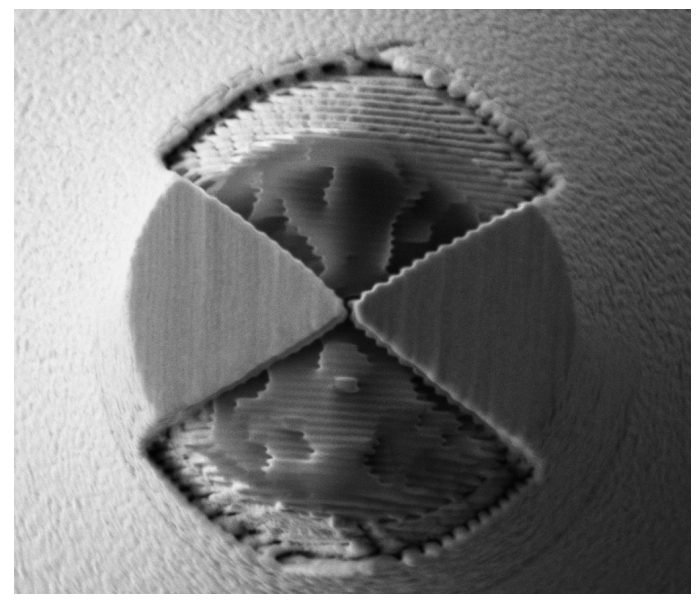

Figure 8.13: Top down view of probe.

from the sample. This light propagates back up the fiber and is roughly collimated by the same objective lens which initially focused it into the fiber. A beam splitter then serves to separate the reflected light from the incident light, which is directed into the PBS detector assembly for Kerr analysis. Beyond this simple modification, only one task remains to be completed: we must design a probe, paying careful consideration to field enhancement, geometry, polarization effects, and feasibility of fabrication. It remains to be seen just how difficult this task will prove. 

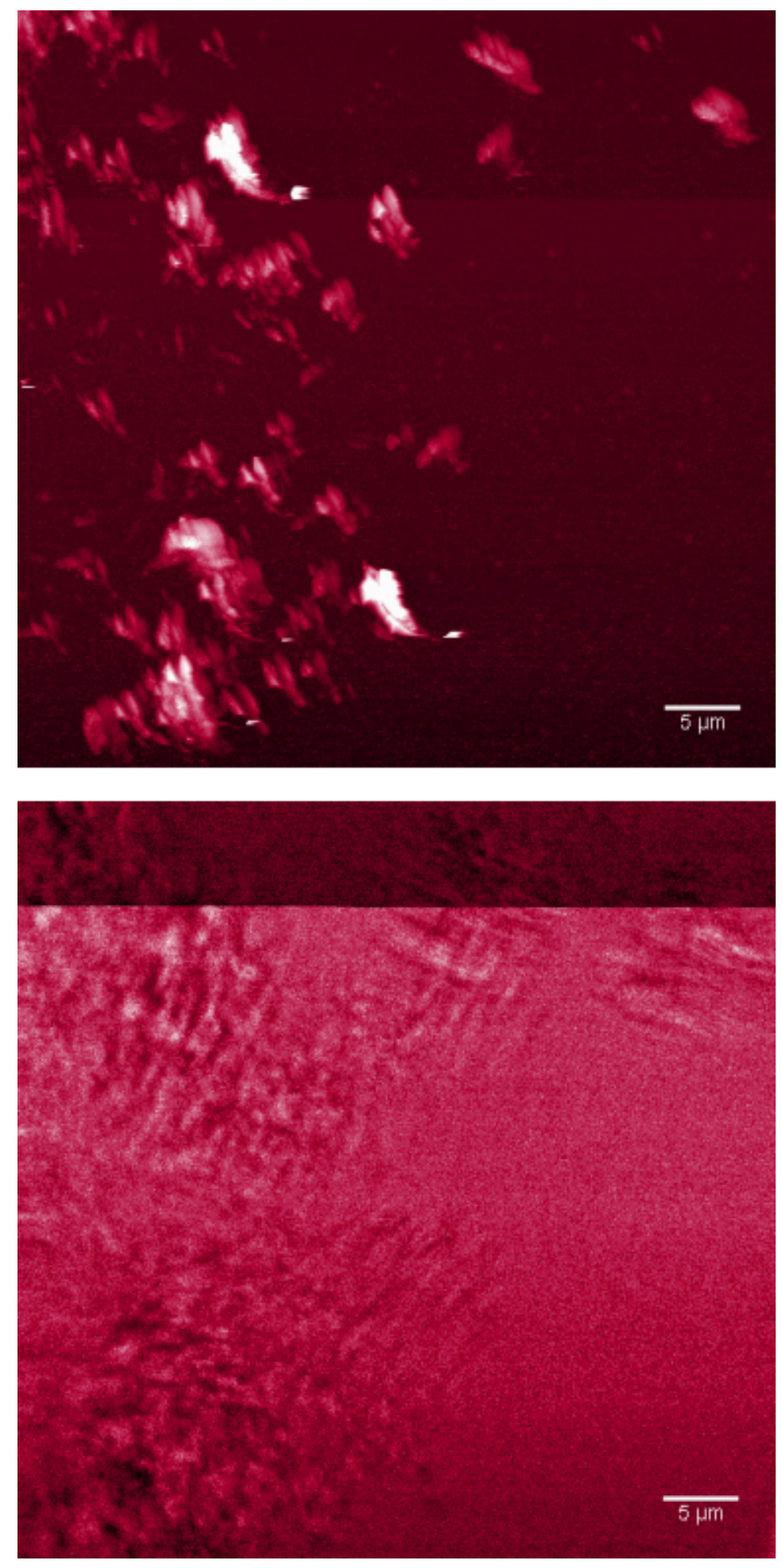

Figure 8.14: Simultaneous topographic (top) and optical transmission (bottom) images of gold film using a pulled optical fiber. 


\section{Conclusions}

We have presented the development of a versatile imaging tool. Chapter 2 gave some background on samples of interest, explaining why we elected to utilize magnetooptical disks as our primary sample.

In chapters 3 and 4 , we discussed the physical development of the microscope. Beginning with a basic schematic for an inverted optical microscope, we used SolidWorks to assemble a functional instrument. Preliminary testing was performed with a CCD camera as the detector. Point scanning capabilities were the next addition, requiring a piezo scan stage, an $\mathrm{APD}$, and the analog and control requirements for scanning and collection. We investigated magnetic glass with great success, obtaining resolution below $400 \mathrm{~nm}$. The microscope was also configured in fluorescence mode as a demonstration of versatility, and we were successful in acquiring fluorescence images of rhodamine $6 \mathrm{G}$. With the addition of a shear force scan head and AFM control systems, the microscope became capable of shear force imaging, which was demonstrated by scanning an AFM calibration grid.

Chapter 5 provided the theoretical framework for the magneto-optic Kerr effect (MOKE), the principle on which the microscope relies for magnetic imaging. The mechanism by which this occurs is discussed in chapter 6. Using a differential detection scheme to separate the s and p components of the reflected light, we have successfully imaged magnetic domains of magneto-optic disks.

Reorientation of magnetic domains is described in chapter 7. By laser heating the sample beyond the Curie temperature and applying an external magnetic field, it should be possible to refreeze domains in the direction of the field, thereby making the microscope capable of reading as well as writing.

Finally, chapter 8 discusses the adaptation of the system to fiber-based microscopy. 
The fabrication of AFM tips from optical fibers is presented. By coupling light into these AFM fiber probes, we obtained simultaneous optical transmission mode and topographic AFM imaging. 


\section{Bibliography}

[1] A. A. Mamun, G. Guo, and C. Bi, Hard Disk Drive: Mechatronics and Control (Automation and Control Engineering). CRC Press, 2006.

[2] "Seagate breaks areal density barrier: Unveils the world's first hard drive featuring 1 terabyte per platter." Press Release, May 2011.

[3] M. Ohtsu, ed., Near-field Nano/Atom Optics and Technology. Springer, 1 ed., 101998.

[4] E. Betzig and J. Trautman, "Near-field magneto-optics and high density data storage.," Applied Physics Letters, vol. 61, no. 2, p. 142, 1992.

[5] M. Mansuripur, The physical principles of magneto-optical recording. Cambridge New York: Cambridge University Press, 1995.

[6] "Metglas frequently asked questions." http://www.metglas.com/faq/.

[7] Metglas, Magnetic Alloy 2714A (cobalt-based) Technical Bulletin, 2009.

[8] J. A. Ewing, Magnetic Induction In Iron And Other Metals - Third Edition Illustrated. Merchant Books, 3rd ed., Jan 2007.

[9] Metglas, Magnetic Alloy 2605SA1 (iron-based) Technical Bulletin, 2009.

[10] Metglas, Magnetic Alloy 2705M (cobalt-based) Technical Bulletin, 2009.

[11] S. Egelkamp, "Imaging of magnetic domains by the kerr effect using a scanning optical microscope," IOPscience, vol. 79, no. 79, pp. 1-6, 1990. 
[12] H. J. Mamin, D. Rugar, J. E. Stern, R. E. Fontana, and P. Kasiraj, "Magnetic force microscopy of thin permalloy films.," Applied Physics Letters, vol. 55, no. 3, p. 318, 1989.

[13] H. J. Hug, B. Stiefel, A. Moser, I. Parashikov, A. Klicznik, D. Lipp, H.-J. Gntherodt, G. Bochi, D. I. Paul, and R. C. OHandley, "Magnetic domain structure in ultrathin cu/ni/cu/si(001) films (invited)," Journal of Applied Physics, vol. 79, no. 8, p. 5609, 1996.

[14] S. Singh, Fundamentals of Optical Engineering. Discovery Publishing House, Apr 2009.

[15] S. H. Gage, The Microscope and Histology. Pt. I.the Microscope and Microscopical Methods [1892]. James W. Queen and Co., Aug.

[16] R. Hooke, Micrographia. General Books LLC, 32010.

[17] I. Morison, Introduction to Astronomy and Cosmology. Wiley, Dec.

[18] J. J. Lister, "On some properties in achromatic object-glasses applicable to the improvement of the microscope," Philosophical Transactions of the Royal Society of London, vol. 120, pp. 187-200, 1830.

[19] G. Cox, ed., Optical Imaging Techniques in Cell Biology. CRC Press, 1 ed., 11 2006.

[20] F. Zernike, "Phase contrast, a new method for the microscopic observation of transparent objects part ii," Physica, vol. 9, no. 10, pp. 974 - 986, 1942.

[21] M. Mansuripur, Classical Optics and its Applications. Cambridge University Press, 2 ed., Mar 2009. 
[22] R. Zsigmondy, Colloids and the Ultramicroscope; A Manual of Colloid Chemistry and Ultramicroscopy. General Books LLC, 2010.

[23] E. Synge, "A suggested method for extending the microscopic resolution into the ultramicroscopic region," Phil. Mag., vol. 6, no. 356, 1928.

[24] E. A. Ash and G. Nicholls, "Super-resolution aperture scanning microscope," Nature, vol. 237, no. 510, 1972.

[25] G. Binnig, H. Rohrer, C. Gerber, and E. Weibel, "Surface studies by scanning tunneling microscopy," Phys. Rev. Lett., vol. 49, pp. 57-61, Jul 1982.

[26] A. Lewis, M. Isaacson, A. Harootunian, and A. Muray, "Development of a 500 å spatial resolution light microscope: I. light is efficiently transmitted through 入/16 diameter apertures," Ultramicroscopy, vol. 13, no. 3, pp. 227 - 231, 1984.

[27] D. W. Pohl, W. Denk, and M. Lanz, "Optical stethoscopy: Image recording with resolution $\lambda / 20$," Applied Physics Letters, vol. 44, pp. 651-653, Apr. 1984.

[28] D. B. Nowak, A. J. Lawrence, and E. J. Sánchez, "Apertureless near-field/far-field cw two-photon microscope for biological and material imaging and spectroscopic applications," Appl. Opt., vol. 49, pp. 6766-6771, Dec 2010.

[29] D. B. Nowak, A. J. Lawrence, Z. K. Dzegede, J. C. Hiester, C. Kim, and E. J. Sánchez, "Field programmable gate array based reconfigurable scanning probe/optical microscope," Rev. Sci. Instrum., vol. 82, p. 103701, Oct 2011.

[30] F. M. Dearmond, "Development of a scanning electrochemical microscope for the detection of gentamicin," Master's thesis, Portland State University, 2011. 
[31] D. B. Nowak, The Design of a Novel Tip Enhanced Near-field Scanning Probe Microscope for Ultra-High Resolution Optical Imaging. PhD thesis, Portland State University, 2010.

[32] Physik Instrumente, P-733.2 P-733.3 XY(Z) Piezo-Nanopositioning Stage Datasheet, 2008.

[33] E. Hecht, Optics (4th Edition). Addison Wesley, 4 ed., Aug 2001.

[34] H. Du, R.-C. A. Fuh, J. Li, L. A. Corkan, and J. S. Lindsey, "Photochemcad: A computer-aided design and research tool in photochemistry," Photochemistry and Photobiology, vol. 68, no. 2, pp. 141-142, 1998.

[35] M. Born and E. Wolf, Principles of Optics: Electromagnetic Theory of Propagation, Interference and Diffraction of Light. Cambridge University Press, 7th ed., 101999.

[36] E. Abbe, "A contribution to the theory of the microscope and the nature of microscopic vision," Archiv fur Mikroskopische Anatomie, 1984.

[37] C. J. Chen, Introduction to Scanning Tunneling Microscopy (Oxford Series in Optical $\& 3$ Imaging Sciences). Oxford University Press, USA, 1993.

[38] G. Binnig, C. F. Quate, and C. Gerber, "Atomic force microscope," Physical Review Letters, vol. 56, pp. 930-933, Mar. 1986.

[39] S. A. Maier, Plasmonics: Fundamentals and Applications. Springer, softcover reprint of hardcover 1st ed. 2007 ed., Oct 2010.

[40] P. Eaton and P. West, Atomic Force Microscopy. Oxford University Press, USA, May 2010. 
[41] K. Karrai and R. D. Grober, "Piezoelectric tip-sample distance control for near field optical microscopes," Applied Physics Letters, vol. 66, pp. 1842-1844, Apr. 1995.

[42] "Ansom project: An open system microscope design created at portland state university, department of physics." http://ansom.research.pdx.edu/.

[43] V. Zavala-Alonso, R. Aguilera-Flores, N. Patino-Marin, G. A.

Martinez-Casatanon, K. J. Anusavice, and J. P. Loyola-Rodriguez, "Nanostructure evaluation of healthy and fluorotic dentin by atomic force microscopy before and after phosphoric acid etching," Dental Materials Journal, vol. 30, no. 4, pp. 546-553, 2011.

[44] A. Hubert and R. Schfer, Magnetic Domains: The Analysis of Magnetic Microstructures. Springer, corrected ed., Nov 2008.

[45] M. Faraday, "On the magnetic affection of light," Philosophical Magazine, vol. 24, no. 249, pp. 153-166, 1846.

[46] M. N. Deeter, A. Rose, and G. W. Day, "Fast sensitive magnetic field sensors based on the faraday effect in yig," vol. LT-8, pp. 1838-1842, Dec 1990.

[47] D. Awschalom, D. Loss, and N. Samarth, eds., Semiconductor Spintronics and Quantum Computation (NanoScience and Technology). Springer, softcover reprint of hardcover 1st ed. 2002 ed., Dec 2010.

[48] J. Kerr, "On rotation of the plane of polarization by reflection from the pole of a magnet," Philosophical Magazine, 1877. 
[49] W. E. Ayrton, Practical electricity; a laboratory and lecture course for first year students of electrical engineering, based on the international definitions of the electrical units. London [etc.]. Cassell and company, limited, 1896., 2010.

[50] J. Jackson, Classical electrodynamics. New York: Wiley, 1999.

[51] J. C. Maxwell, "On physical lines of force," Philosophical Magazine, vol. 90, no. sup1, pp. 11-23, 2010.

[52] Y. Martin and H. K. Wickramasinghe, "Magnetic imaging by "force microscopy" with 1000 Å resolution," Applied Physics Letters, vol. 50, pp. 1455-1457, May 1987.

[53] K. Bessho, Y. Iwasaki, and S. Hashimoto, "Fabricating nanoscale magnetic mounds using a scanning probe microscope.," Journal of Applied Physics, vol. 79 , no. 8 , p. 5057, 1996.

[54] O. Cespedes, A. Luu, F. Rhen, and J. Coey, "Fabrication of magnetic force microscopy tips via electrodeposition and focused ion beam milling," Magnetics, IEEE Transactions on, vol. 44, pp. 3248 -3251, Nov. 2008.

[55] J. Goldstein, D. E. Newbury, D. C. Joy, C. E. Lyman, P. Echlin, E. Lifshin, L. Sawyer, and J. Michael, Scanning Electron Microscopy and X-ray Microanalysis. Springer, 3rd ed., Feb 2003.

[56] D. C. Joy, "Scanning electron microscopy: Second best no more.," Nat Mater, vol. 8, no. 10, pp. 776-7, 2009.

[57] R. Erni, M. D. Rossell, C. Kisielowski, and U. Dahmen, "Atomic-resolution imaging with a sub-50-pm electron probe," Phys. Rev. Lett., vol. 102, p. 096101, Mar 2009. 
[58] S. McVitie, J. Chapman, L. Zhou, L. Heyderman, and W. Nicholson, "In-situ magnetising experiments using coherent magnetic imaging in tem," Journal of Magnetism and Magnetic Materials, vol. 148, no. 1-2, pp. 232 - 236, 1995.

[59] K. Koike and K. Hayakawa, "Spin polarization of electron-excited secondary electrons from a permalloy polycrystal," Japanese Journal of Applied Physics, vol. 23, no. Part 2, No. 2, pp. L85-L87, 1984.

[60] A. Berger and H. P. Oepen, "Magnetic domain structure in ultrathin cobalt films," Journal of Magnetism and Magnetic Materials, vol. 93, no. 0, pp. 116 $122,1991$.

[61] B. Freeman, M.R.Choi, "Advances in magnetic microscopy.," Science, vol. 294, no. 5546, p. 1484, 2001.

[62] R. Carey, Magnetic domains and techniques for their observation,. English Universities P, 1966.

[63] D. Lukas, "On solution to an optimal shape design problem in 3-dimensional linear magnetostatics," Applications of Mathematics, vol. 49, pp. 441-464, 2004. 10.1023/B:APOM.0000048122.27970.19.

[64] D. Griffiths, Introduction to electrodynamics. Upper Saddle River, N.J: Prentice Hall, 1999.

[65] A. Lauer, "Magnetic field control in three dimensions using visual and numerical vectorization in a software interface," Master's thesis, Portland State University, 2011. 
[66] E. J. Sánchez, L. Novotny, and X. S. Xie, "Near-field fluorescence microscopy based on two-photon excitation with metal tips," Phys. Rev. Lett., vol. 82, pp. 4014-4017, May 1999.

[67] J. J. Doughty, "Near-field probe design: A comparison of symmetric and antisymmetric probesnear-field probe design: A comparison of symmetric and antisymmetric probes," Master's thesis, Portland State University, 2011. 


\section{Appendix - CAD Drawings}

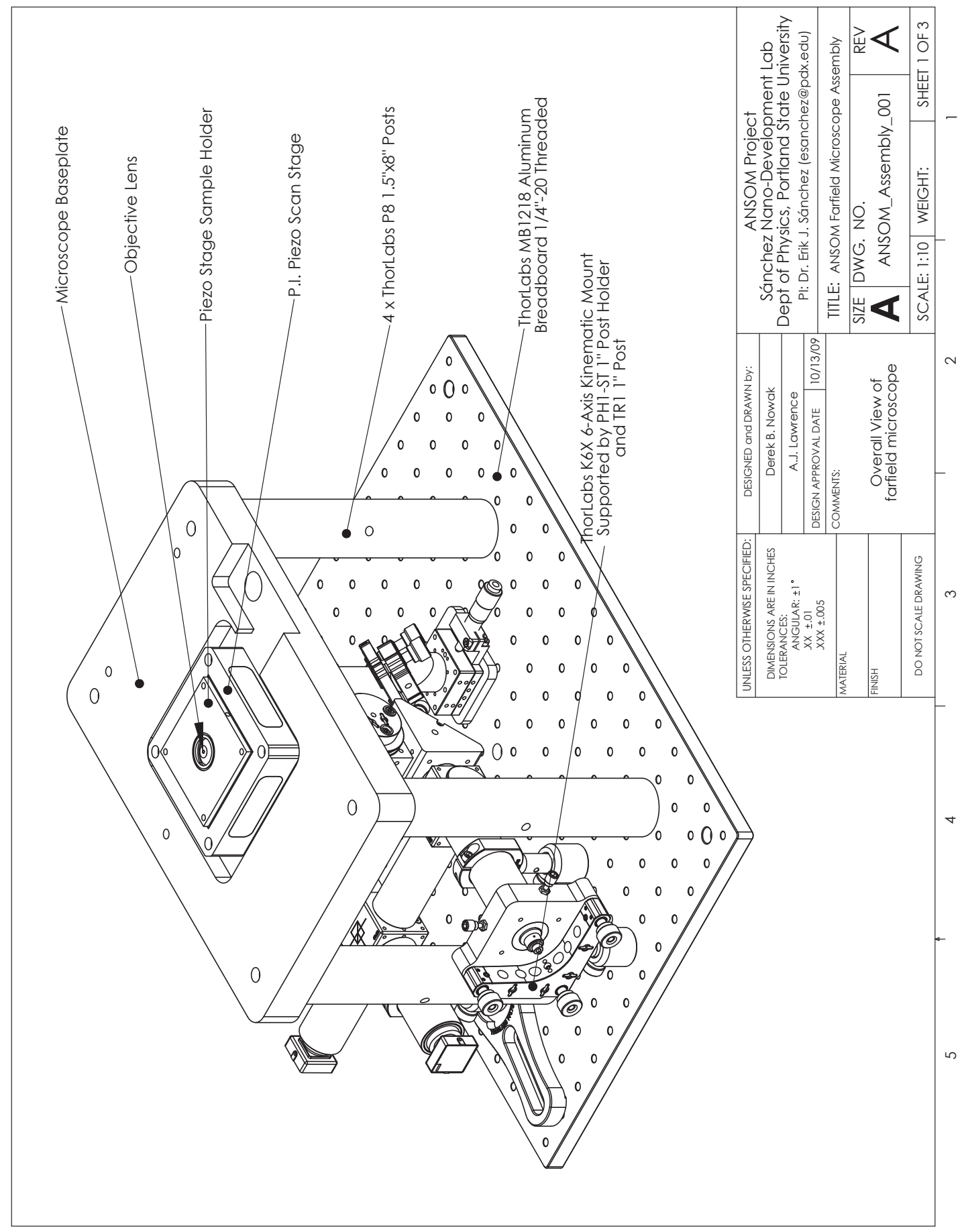




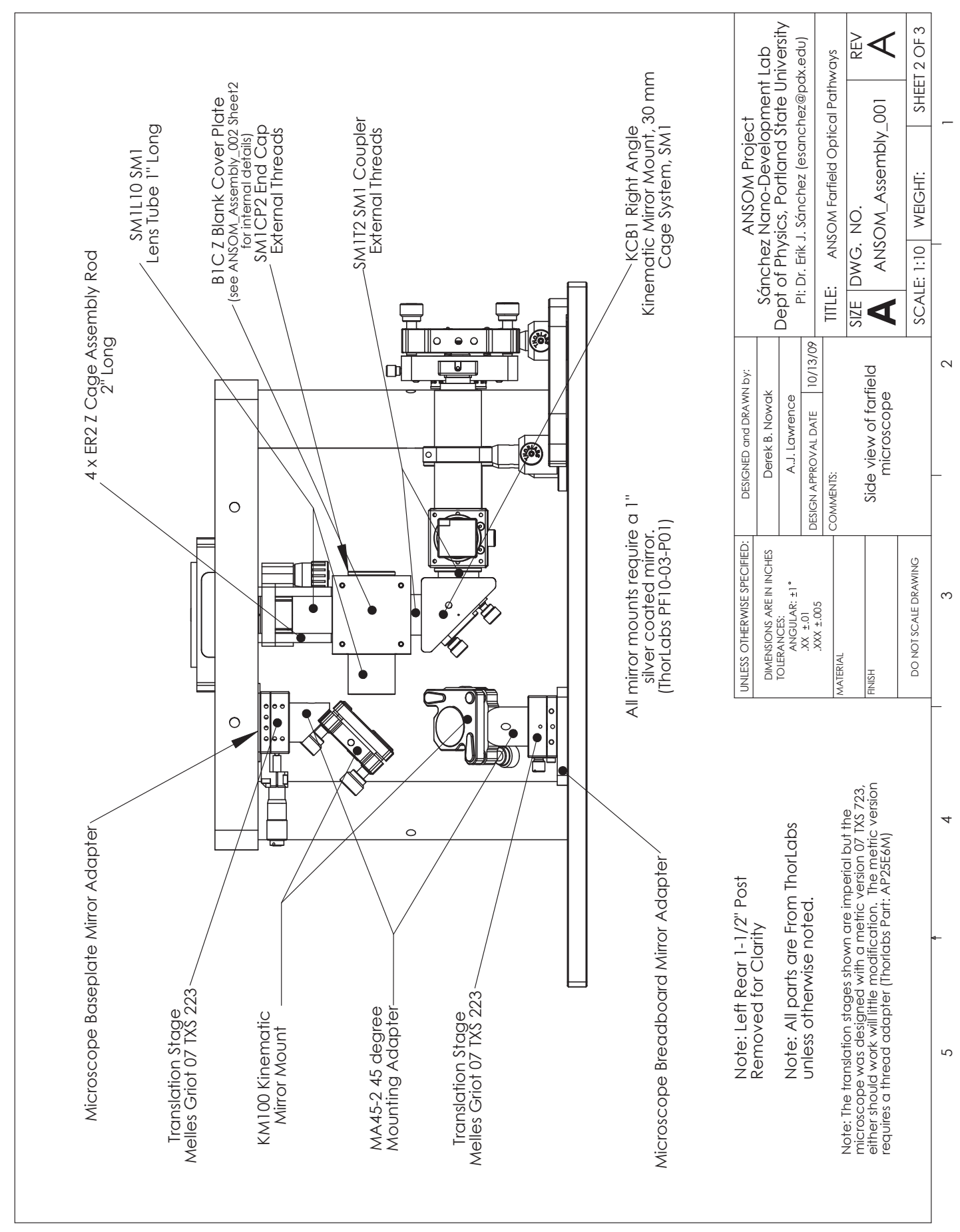




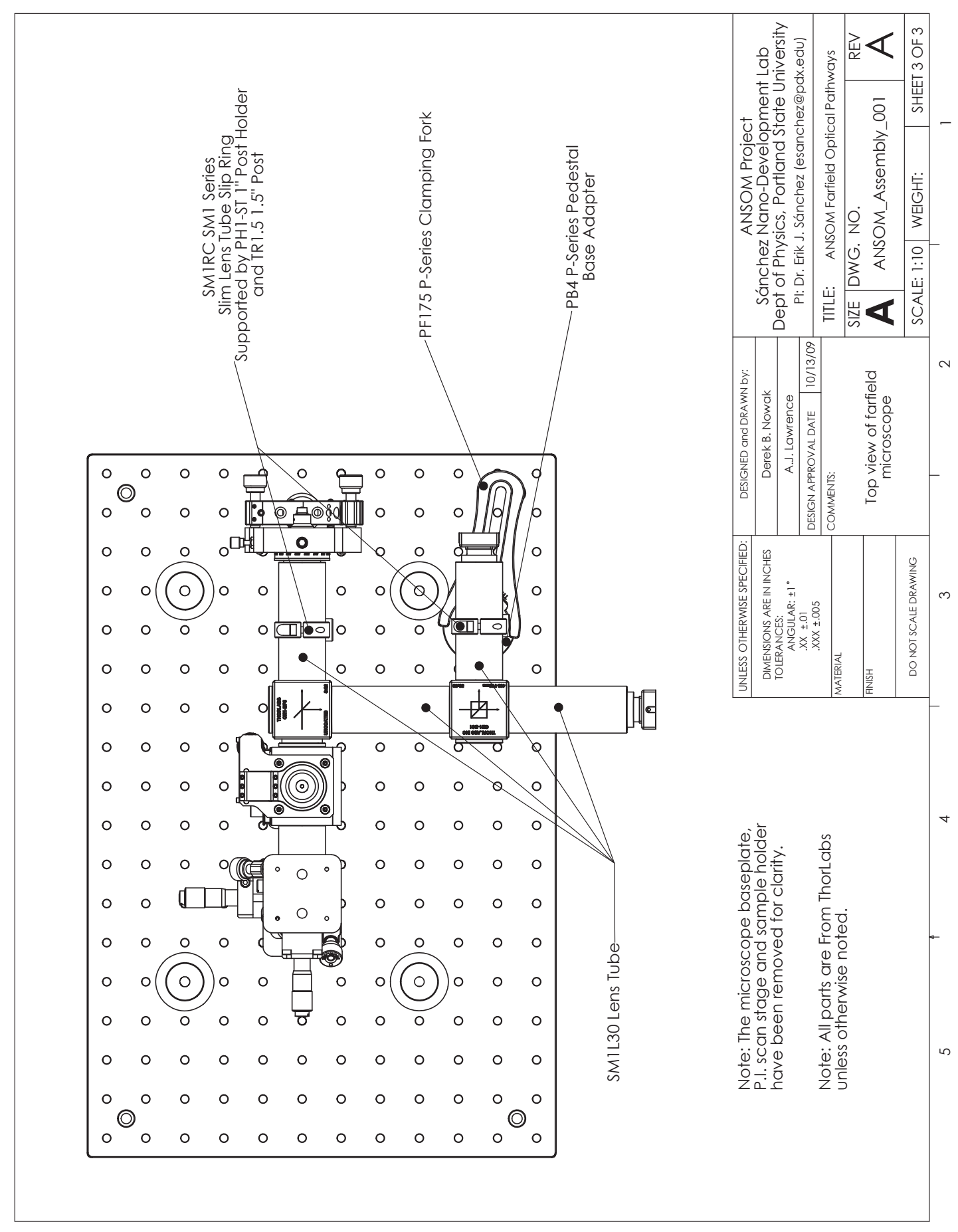




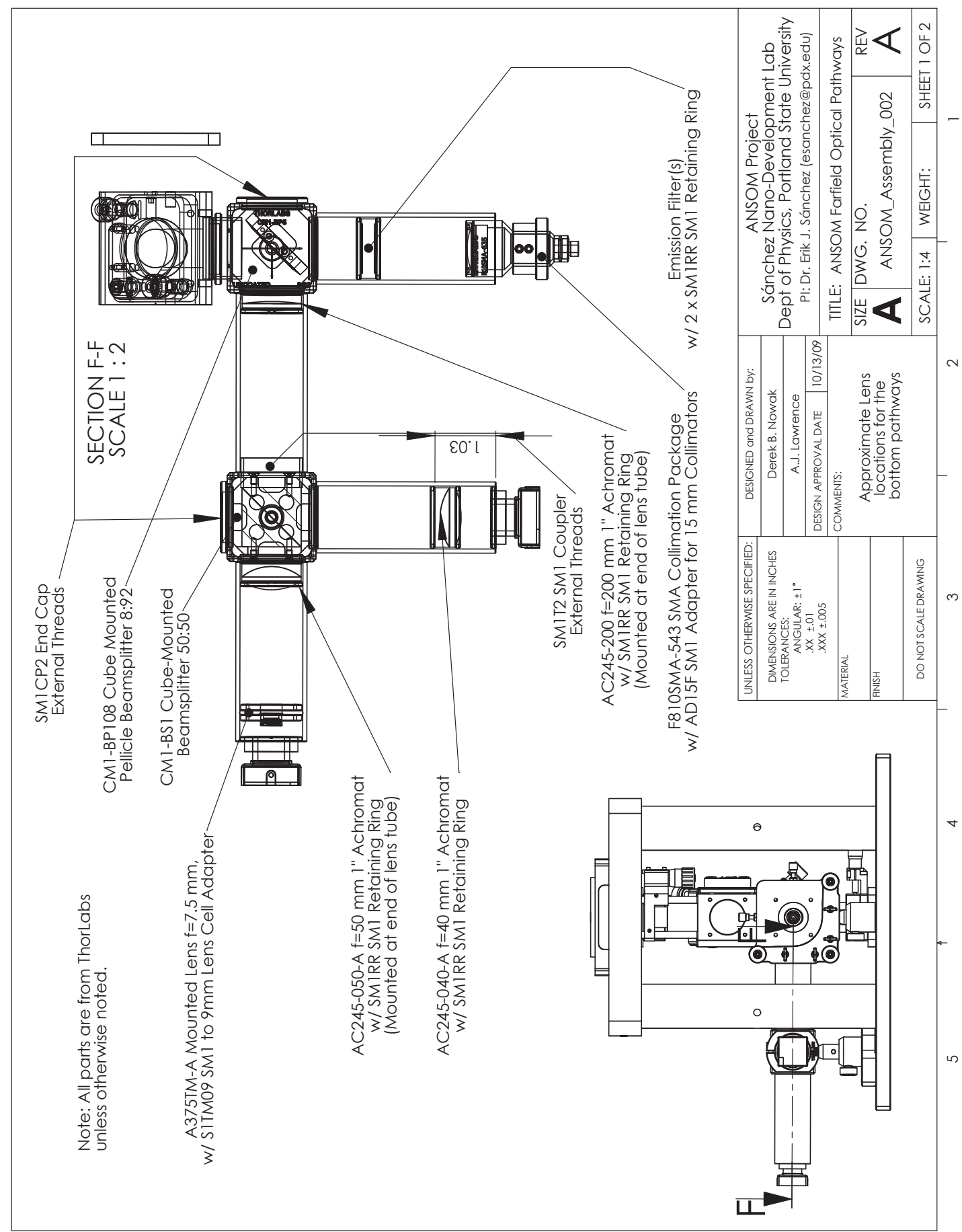




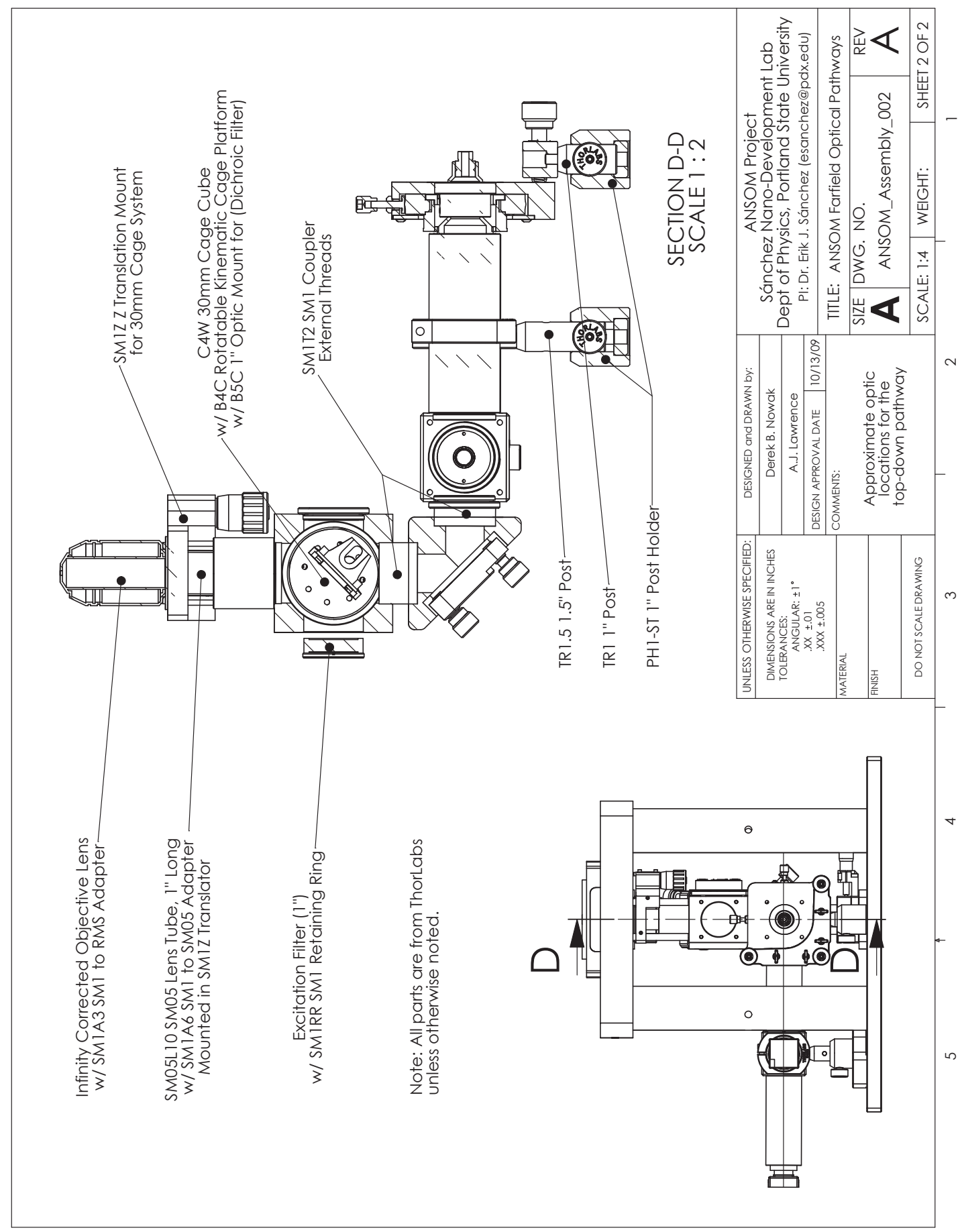




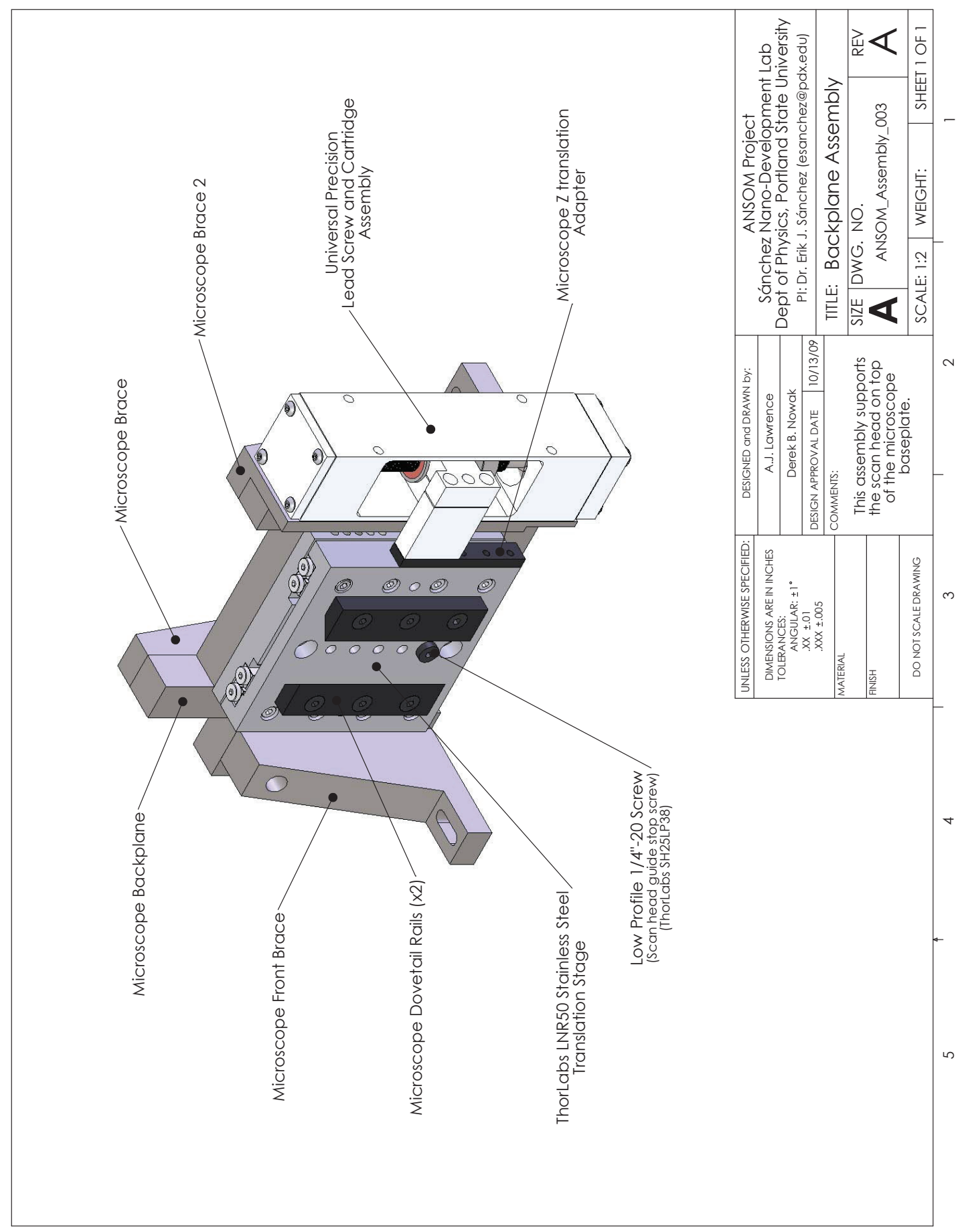




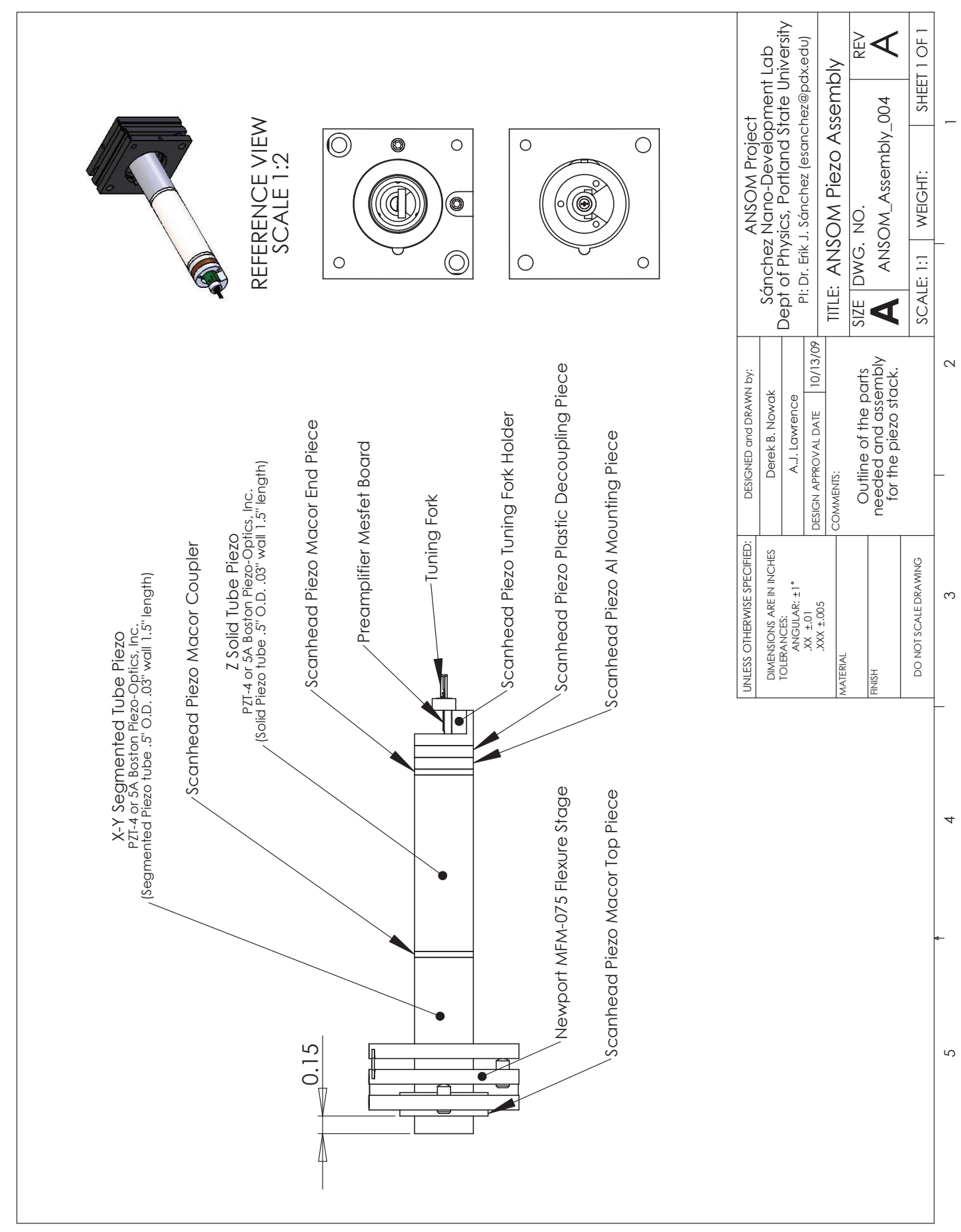




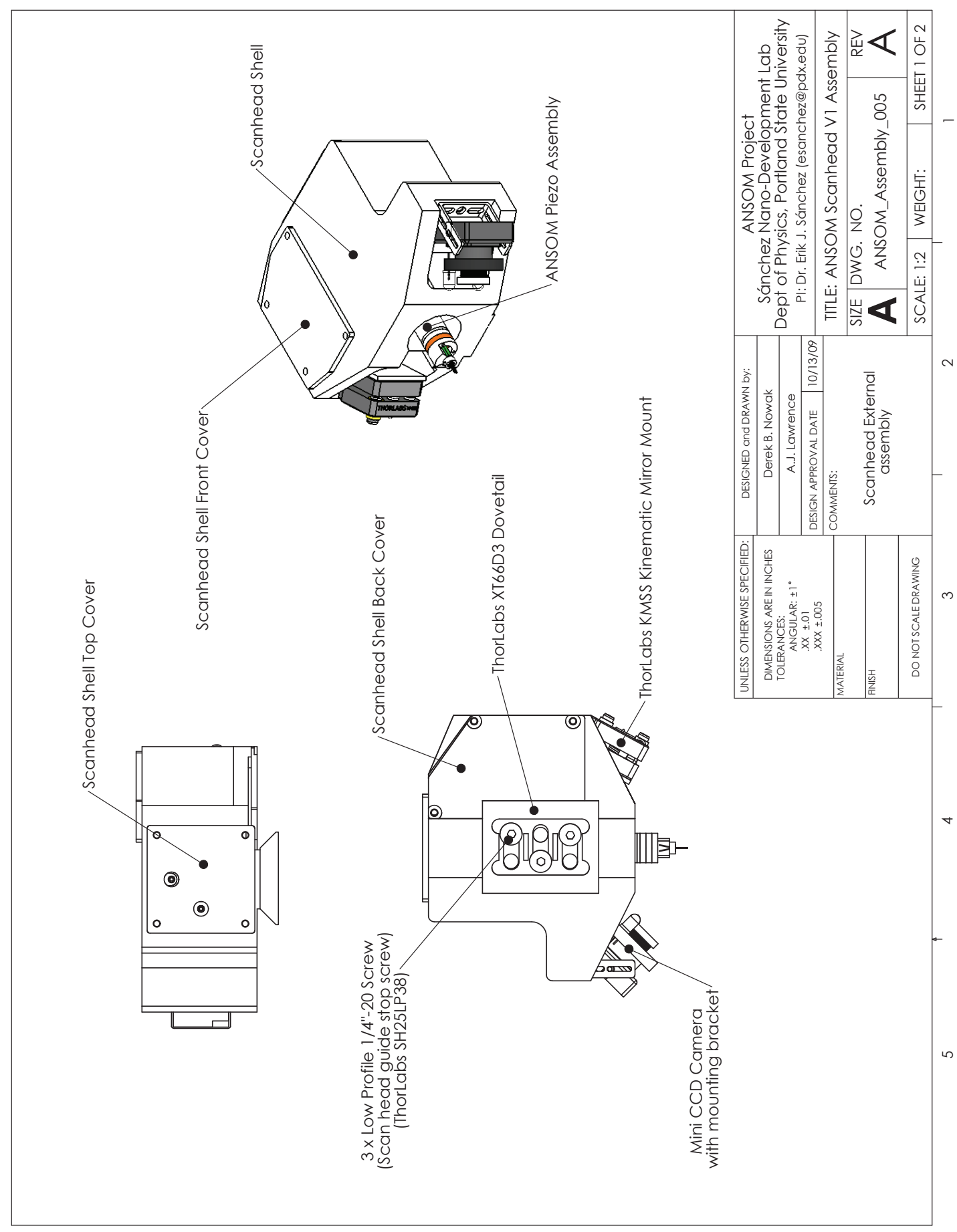




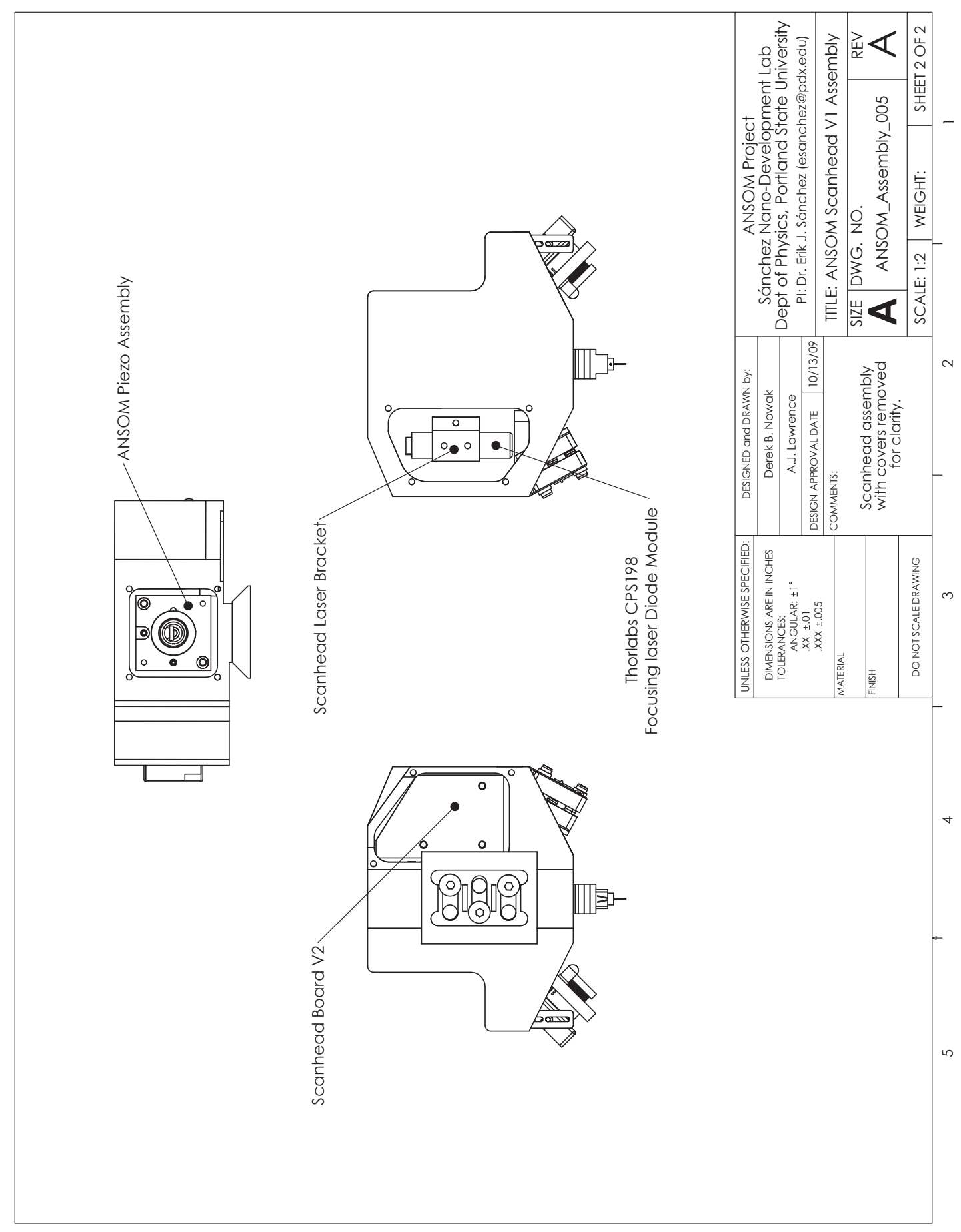




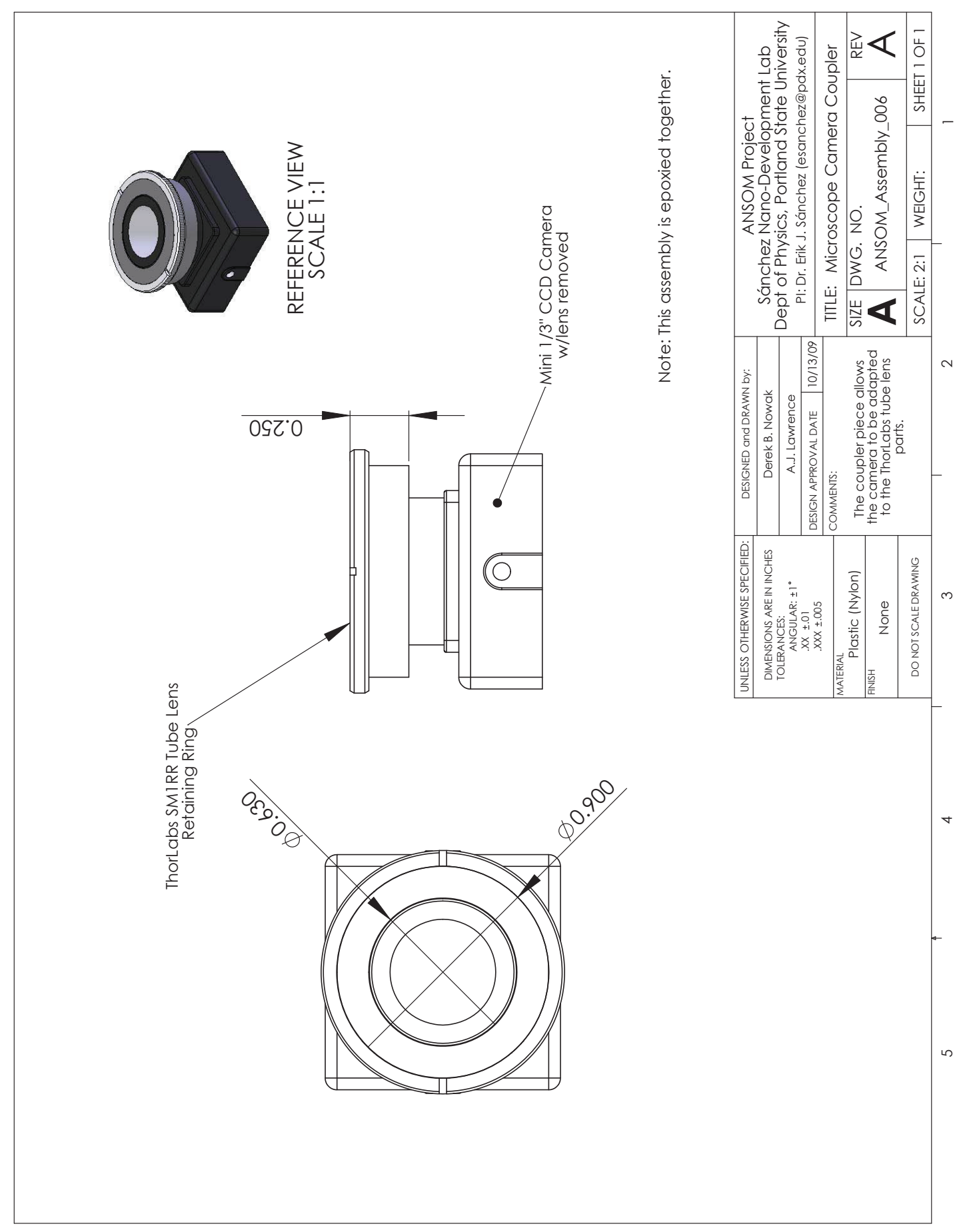




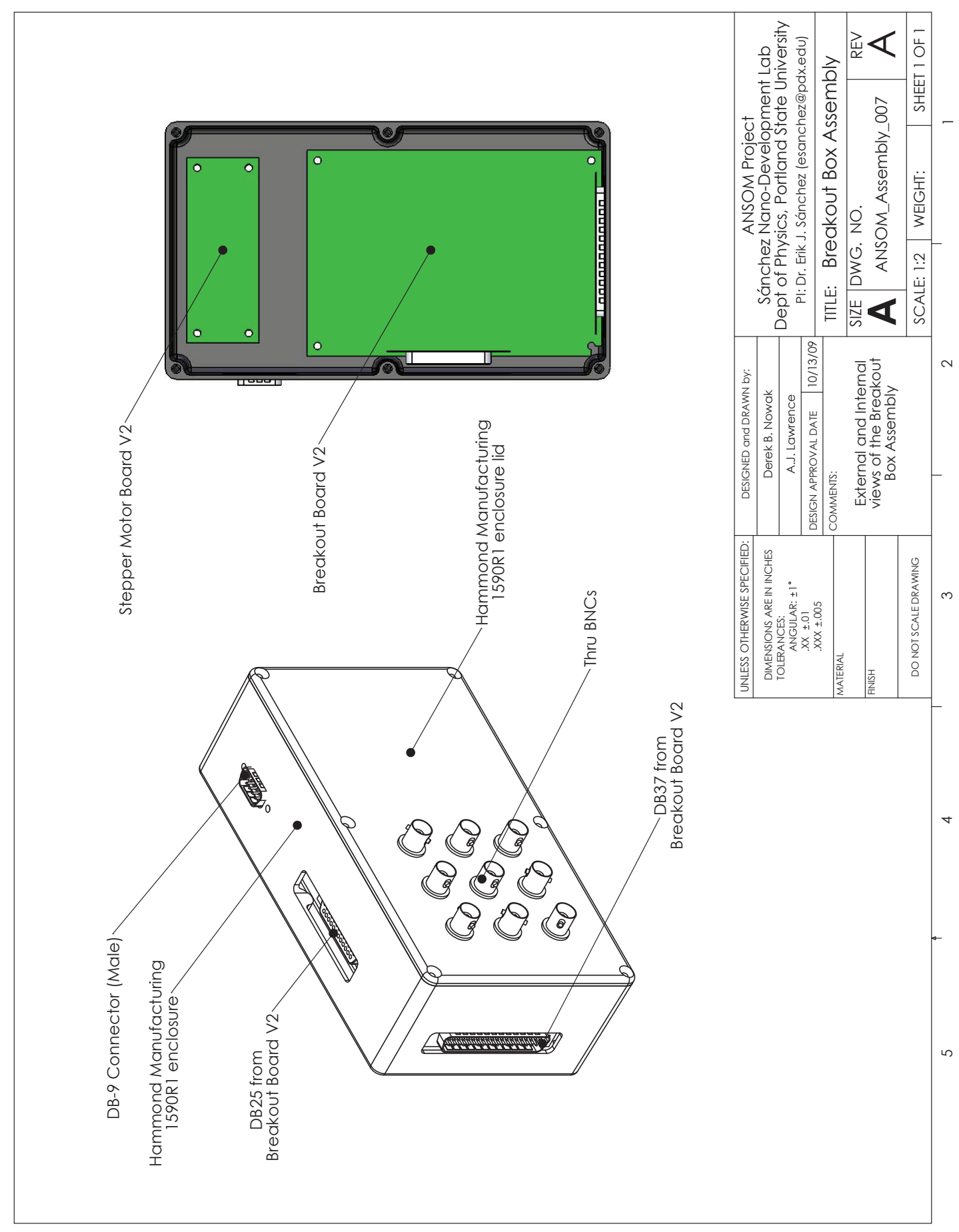




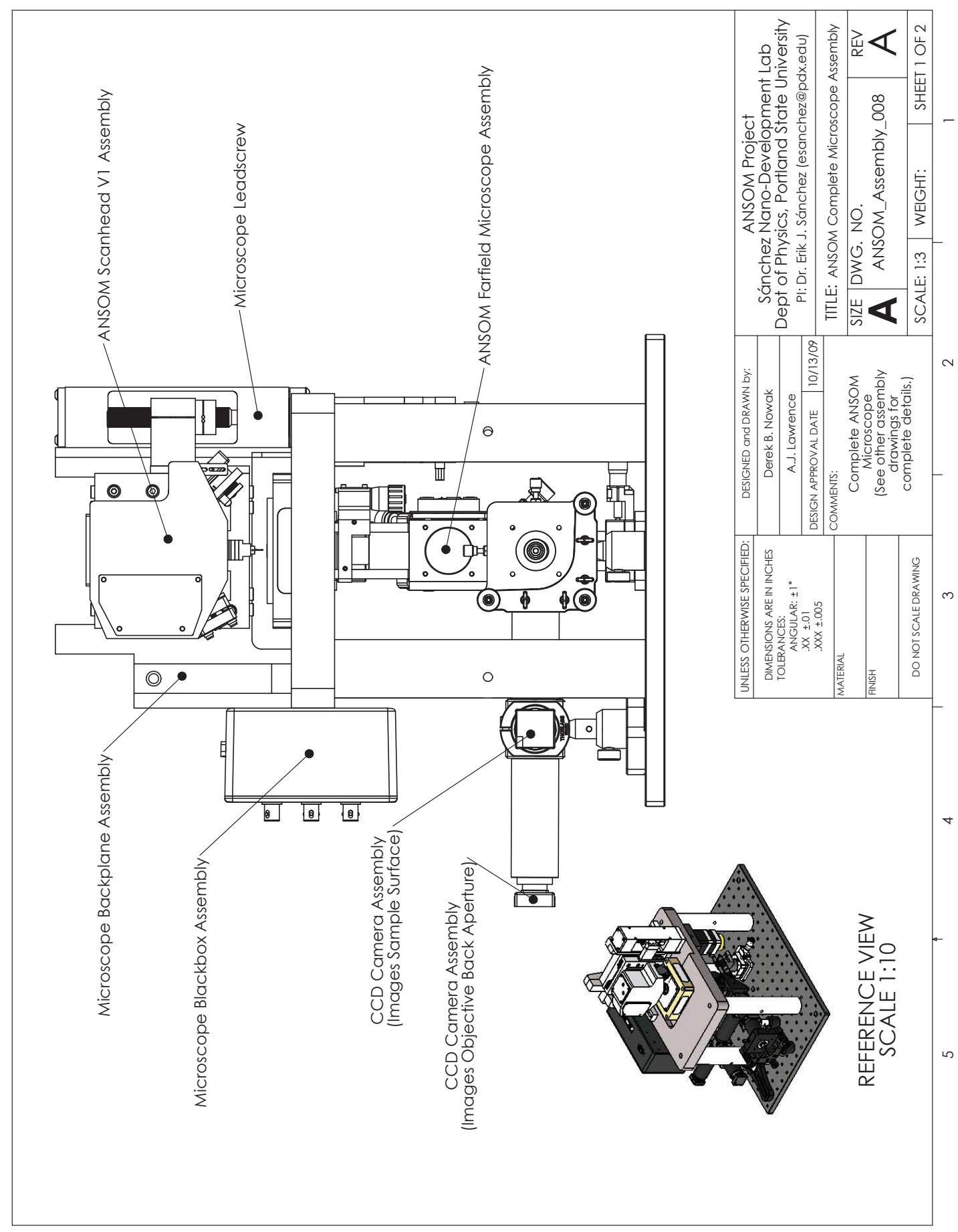




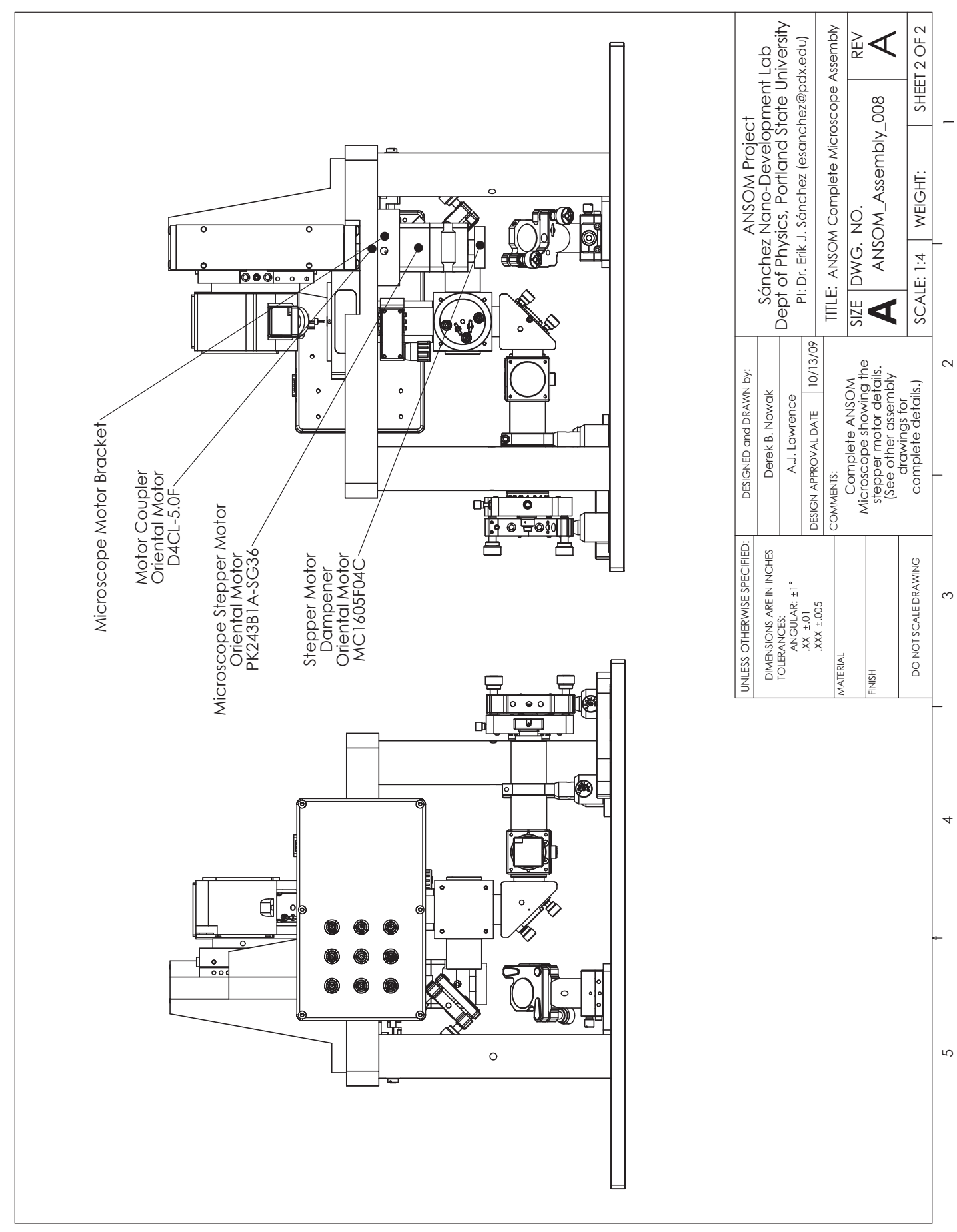




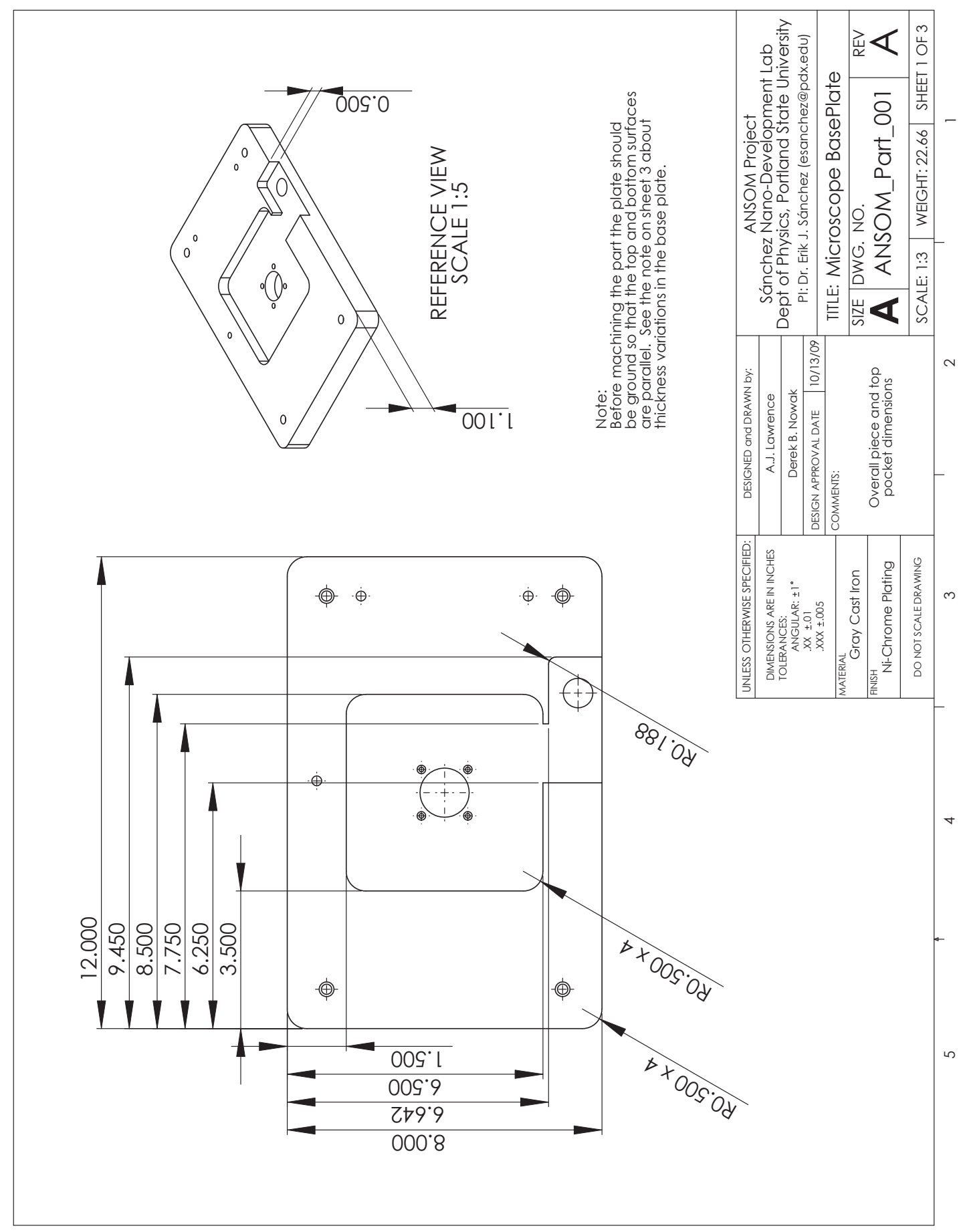




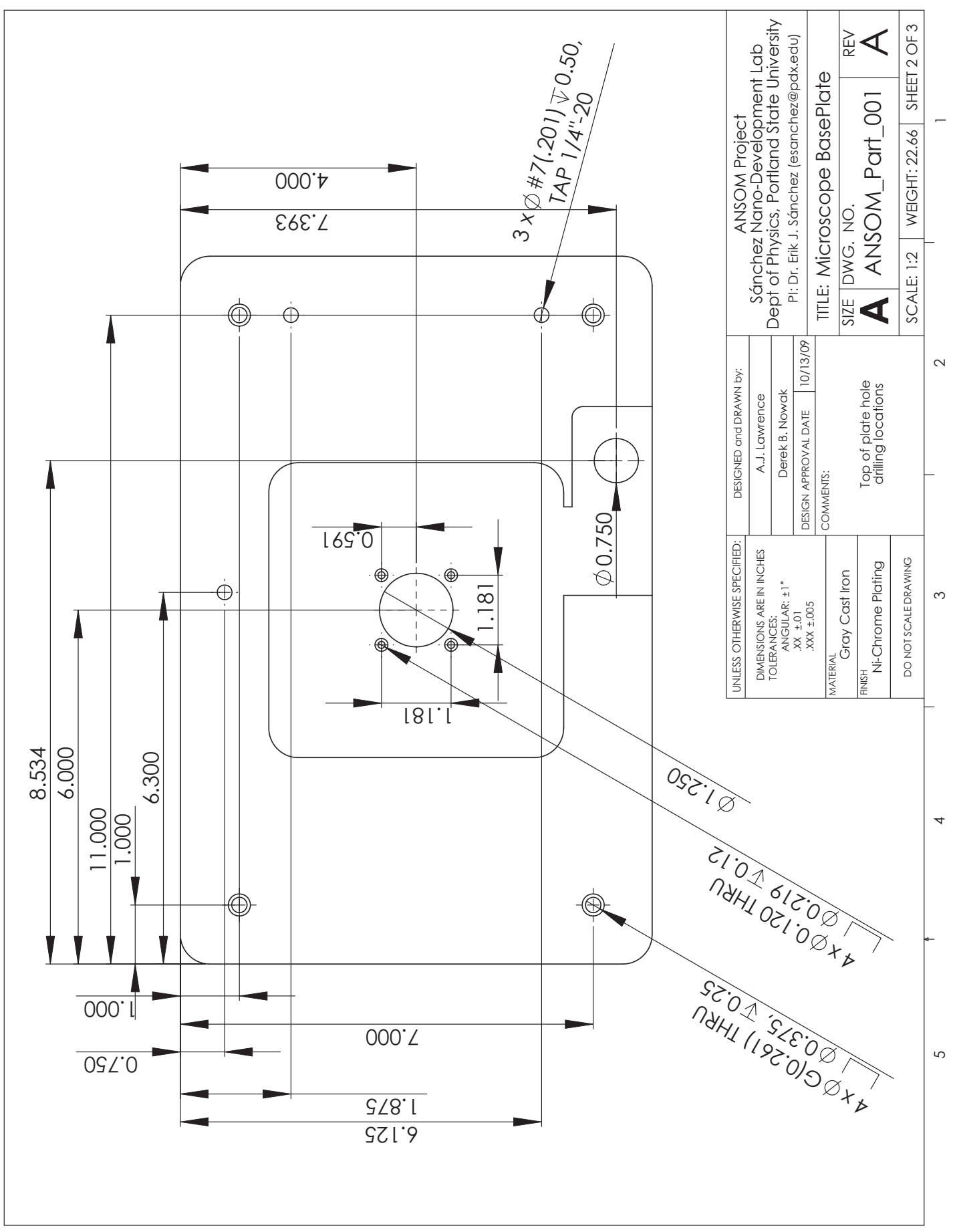




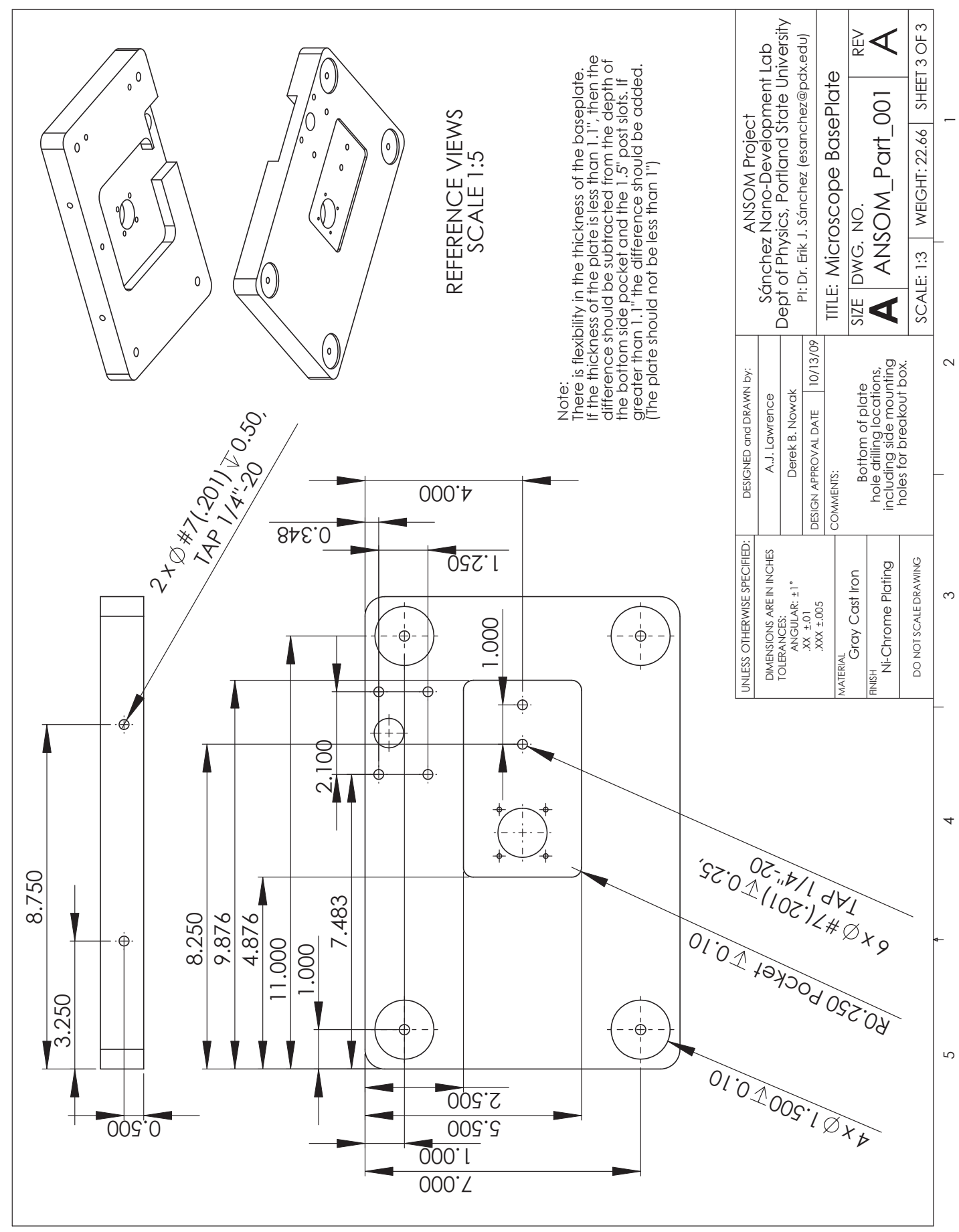




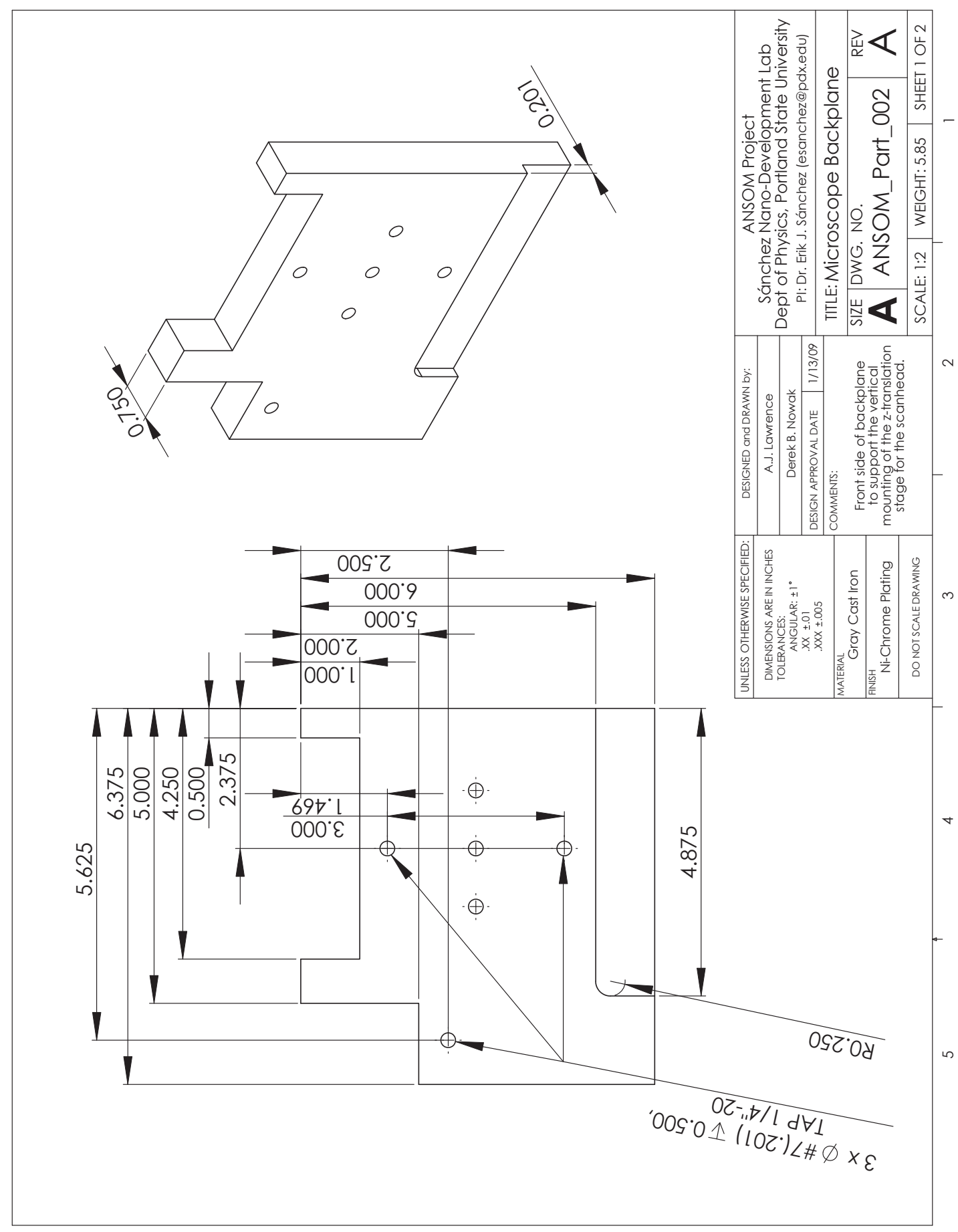




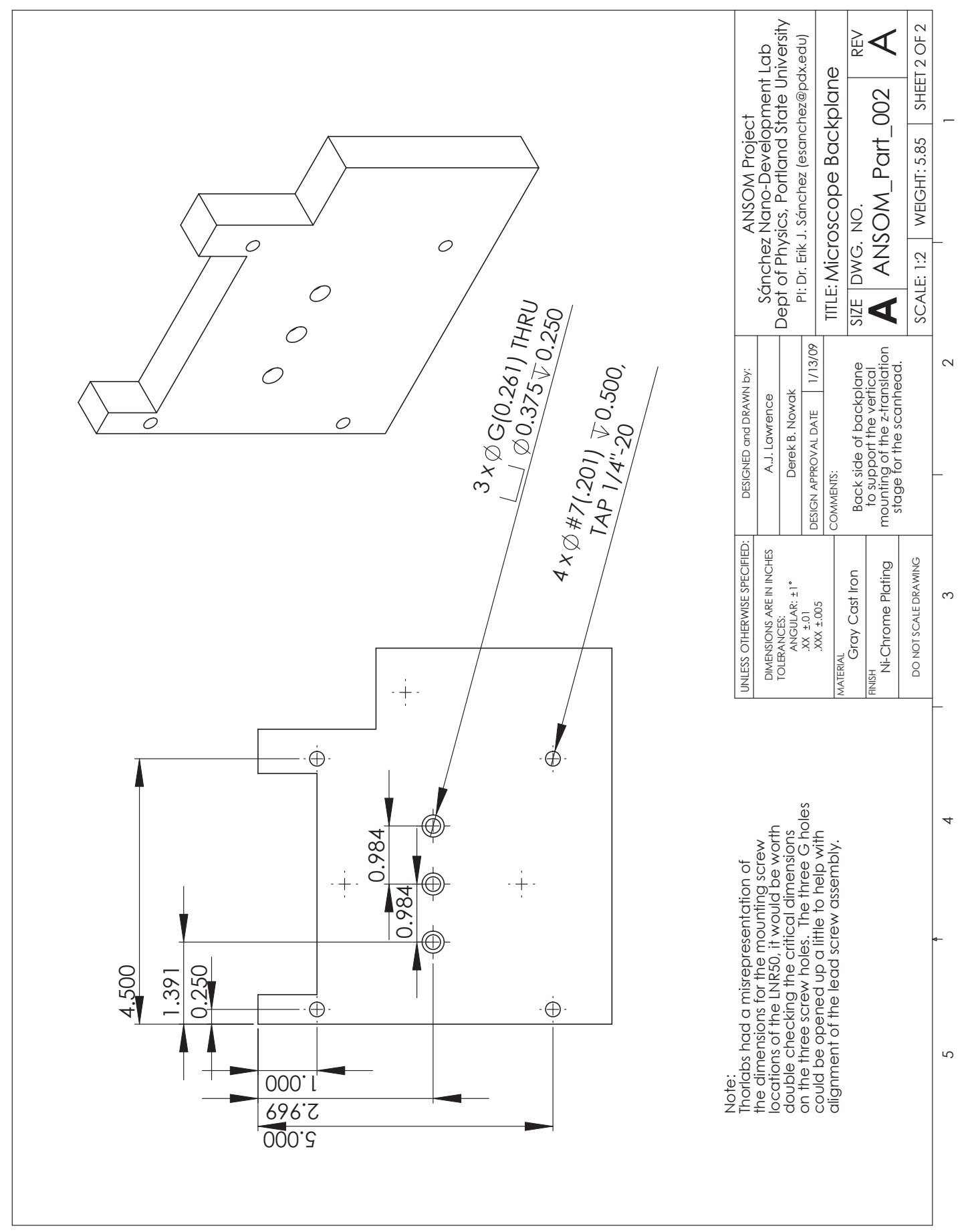




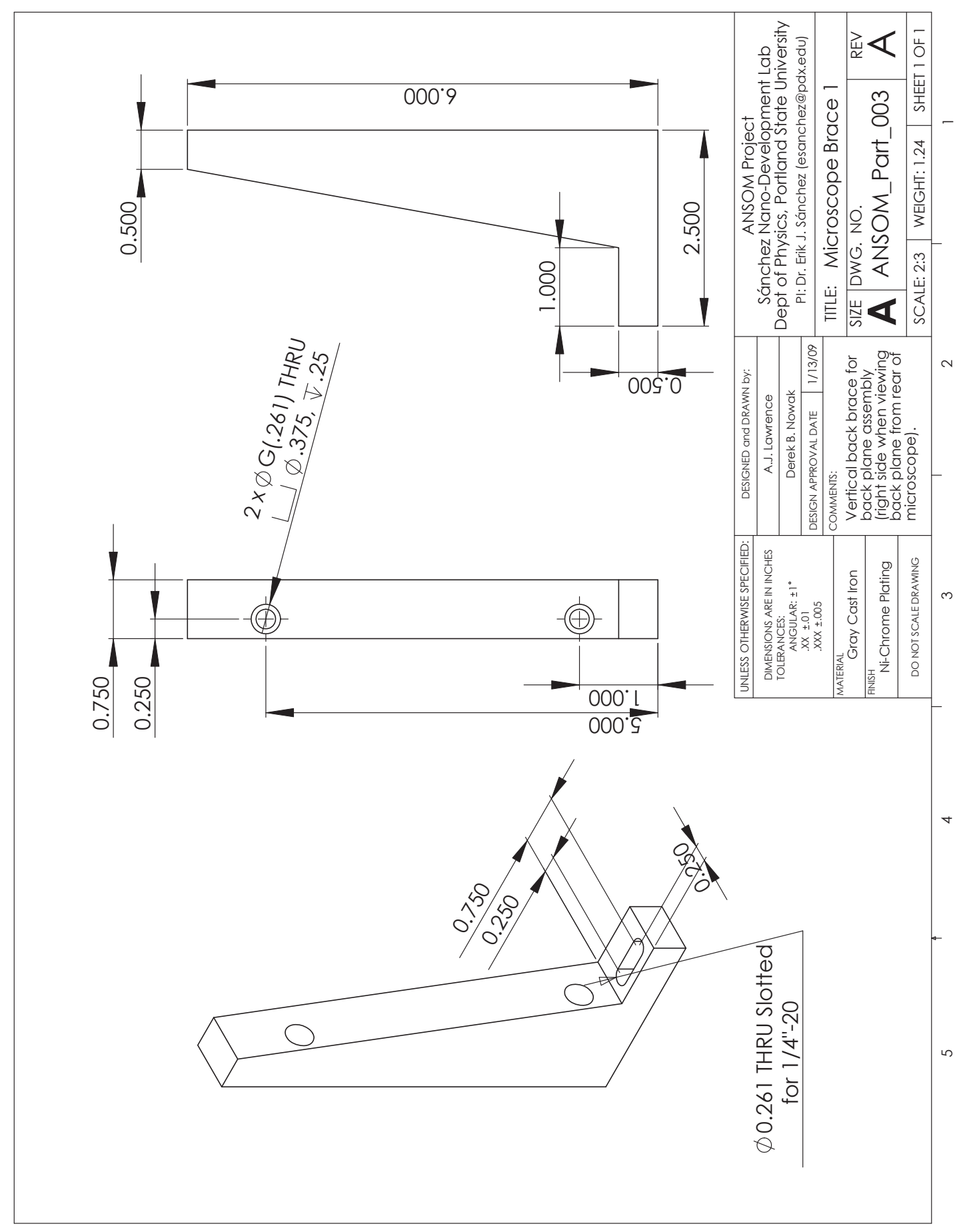




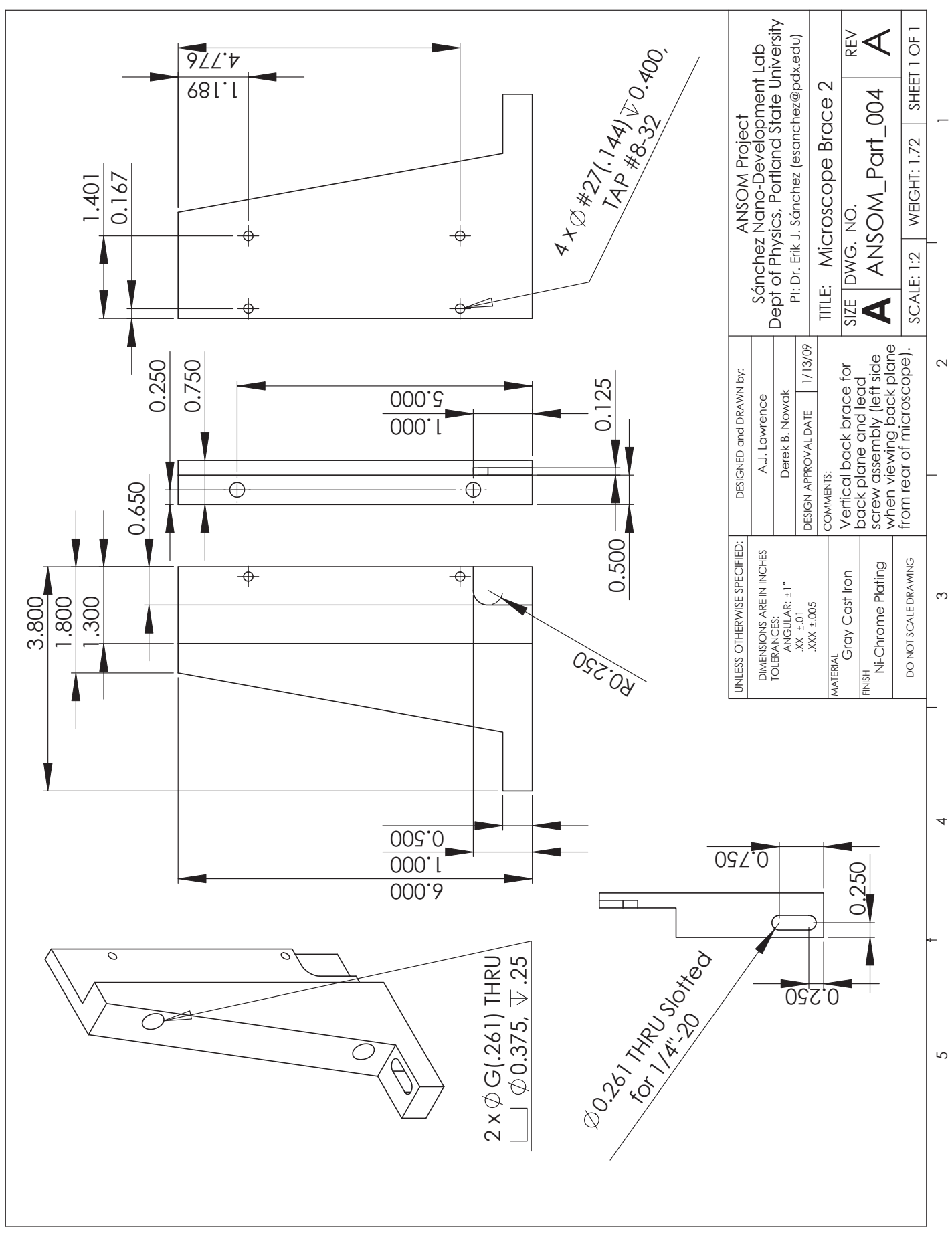




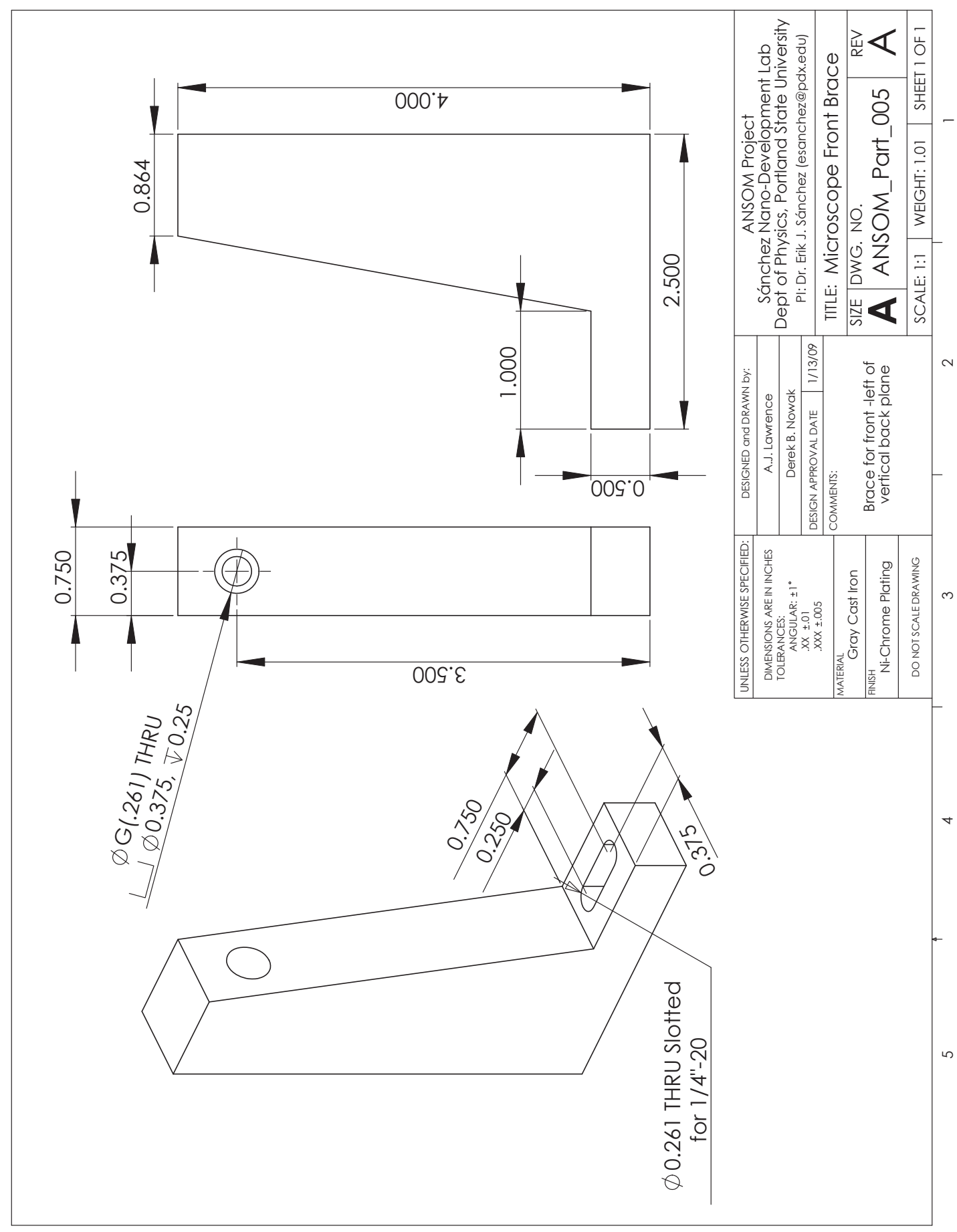




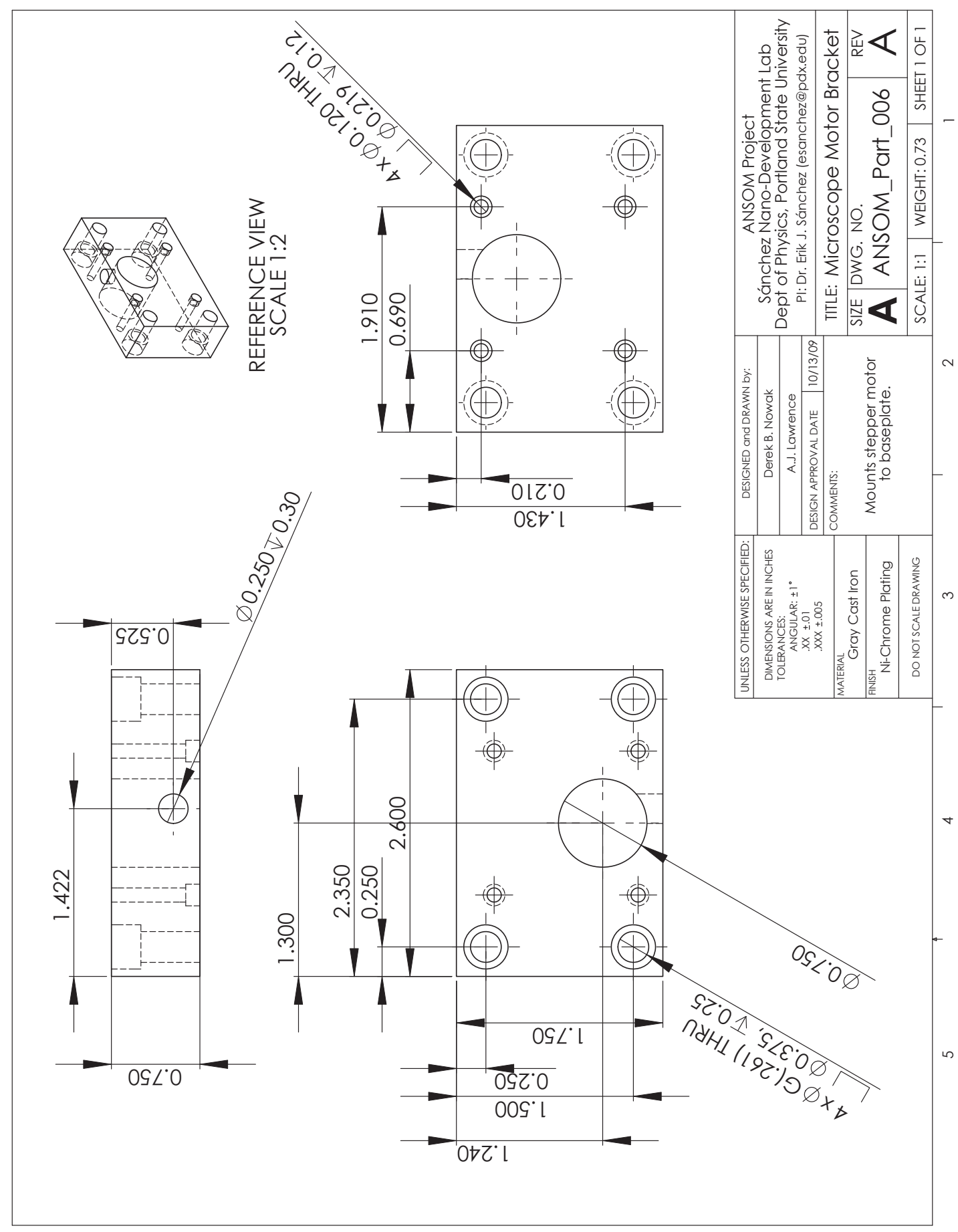




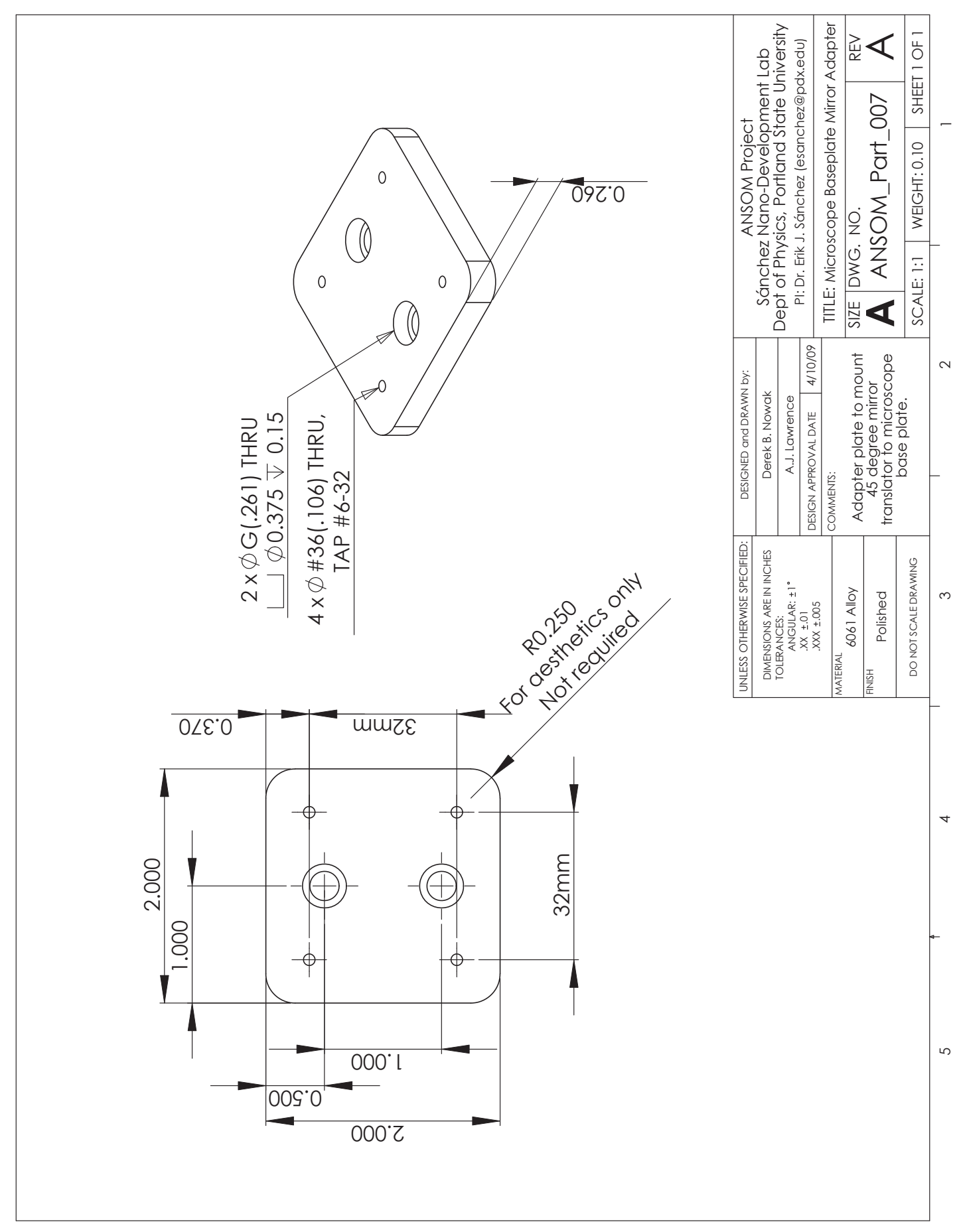




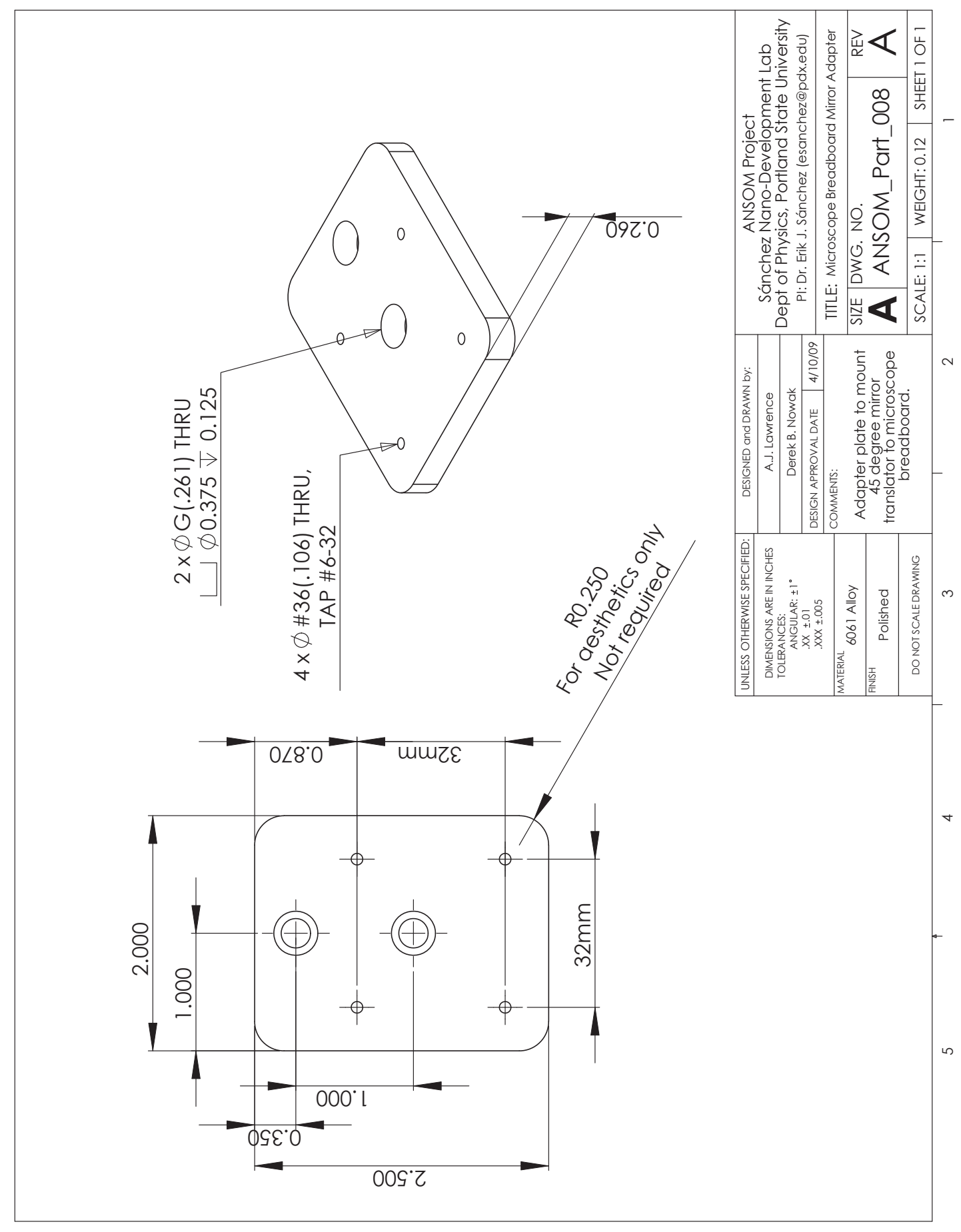




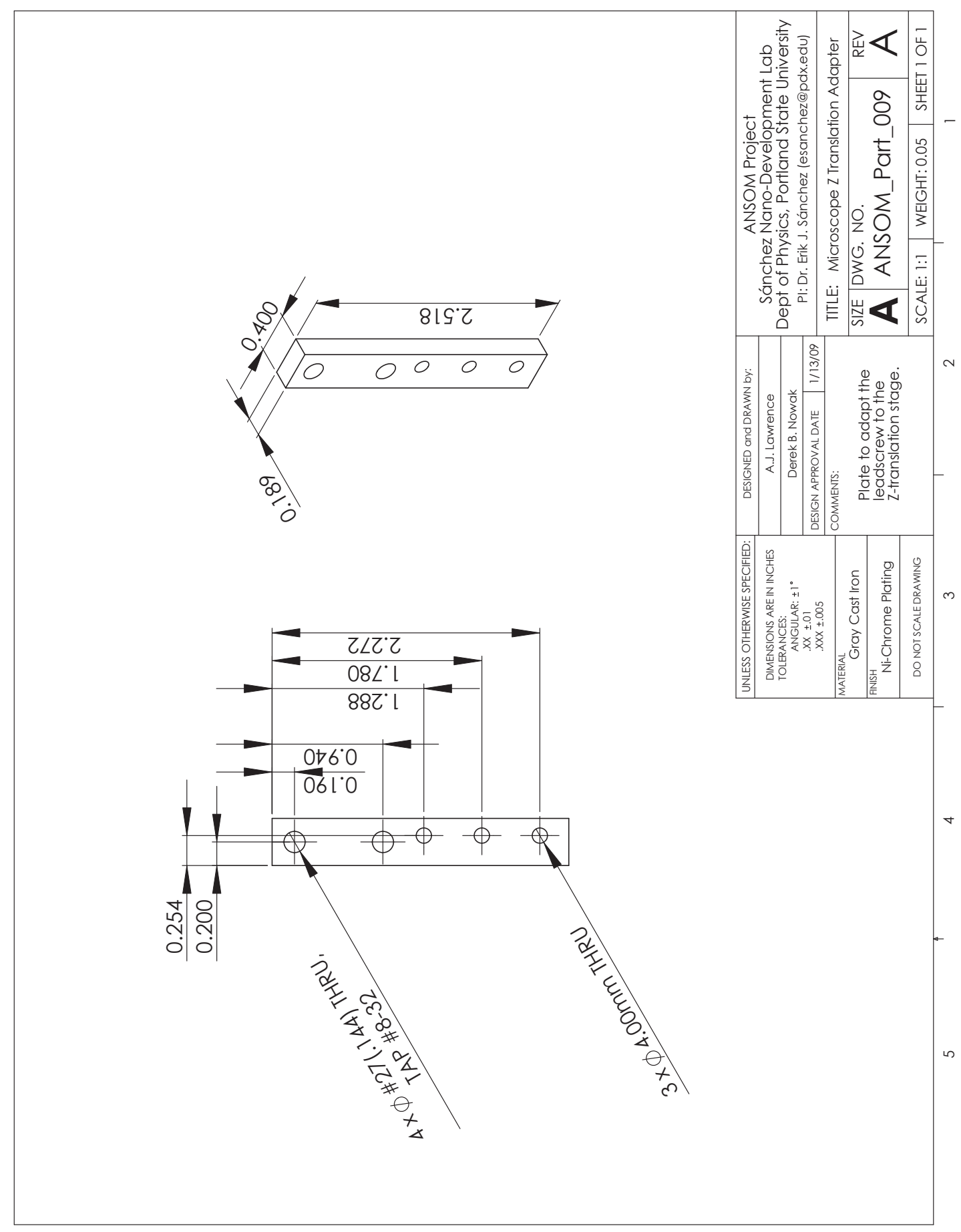




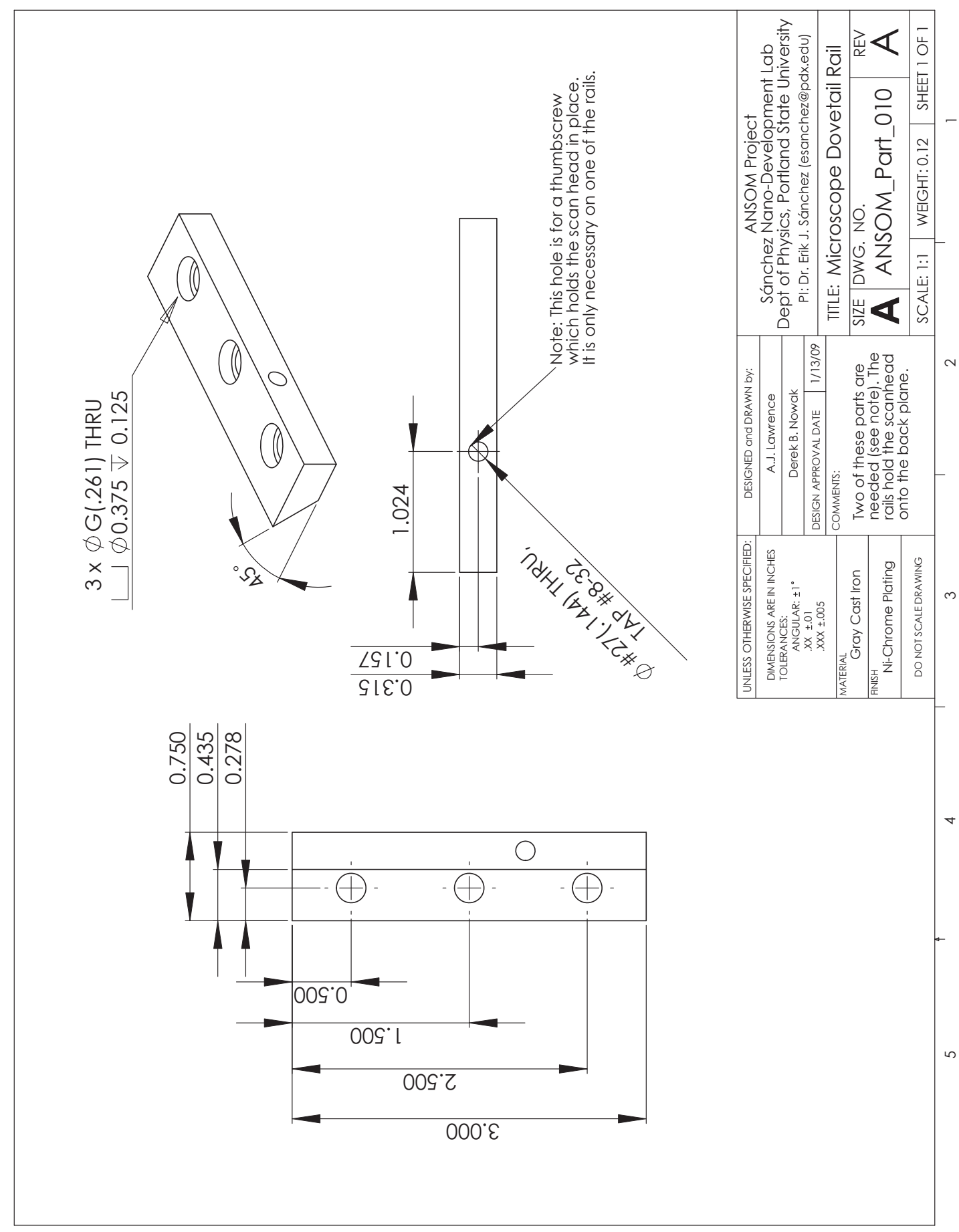




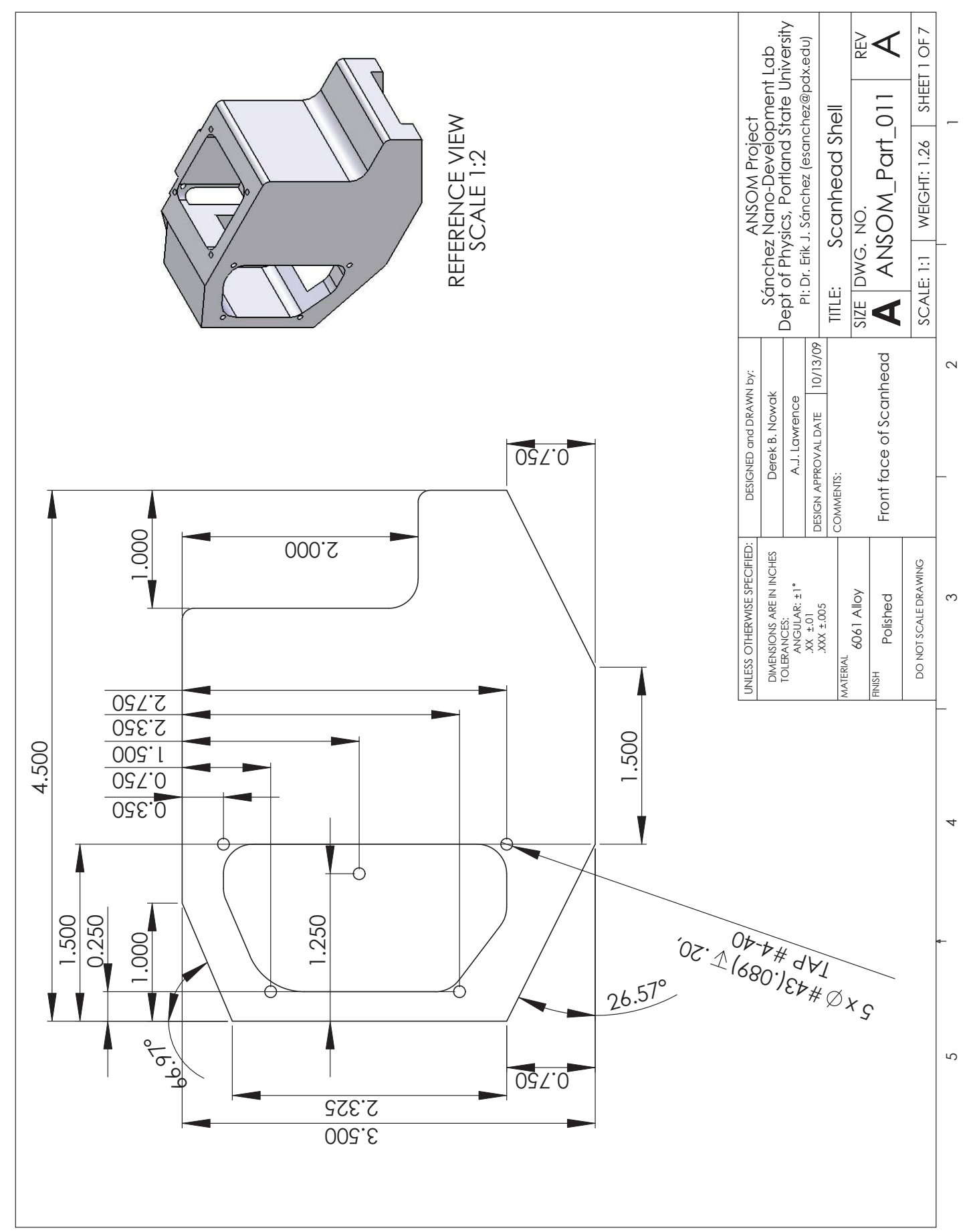




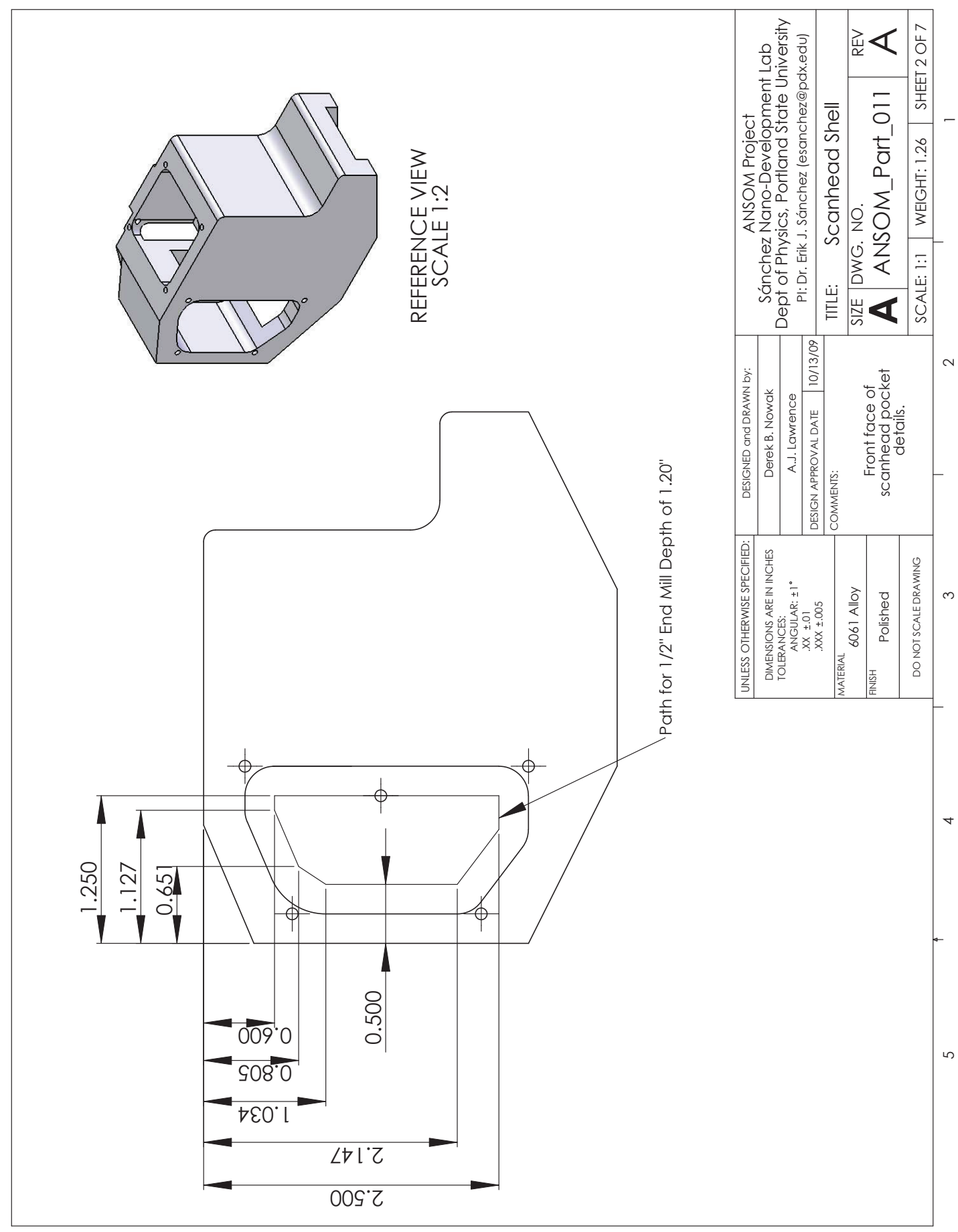




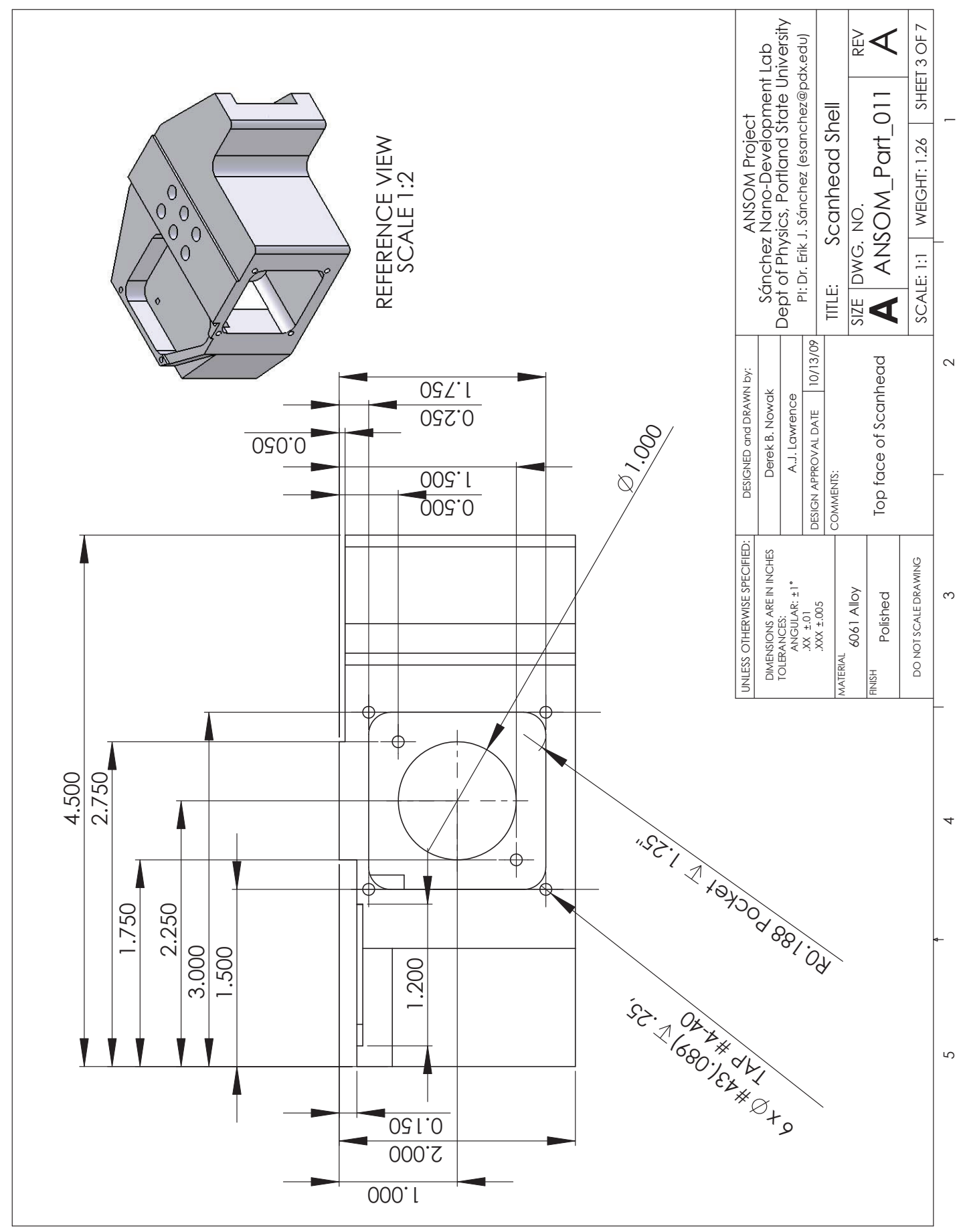




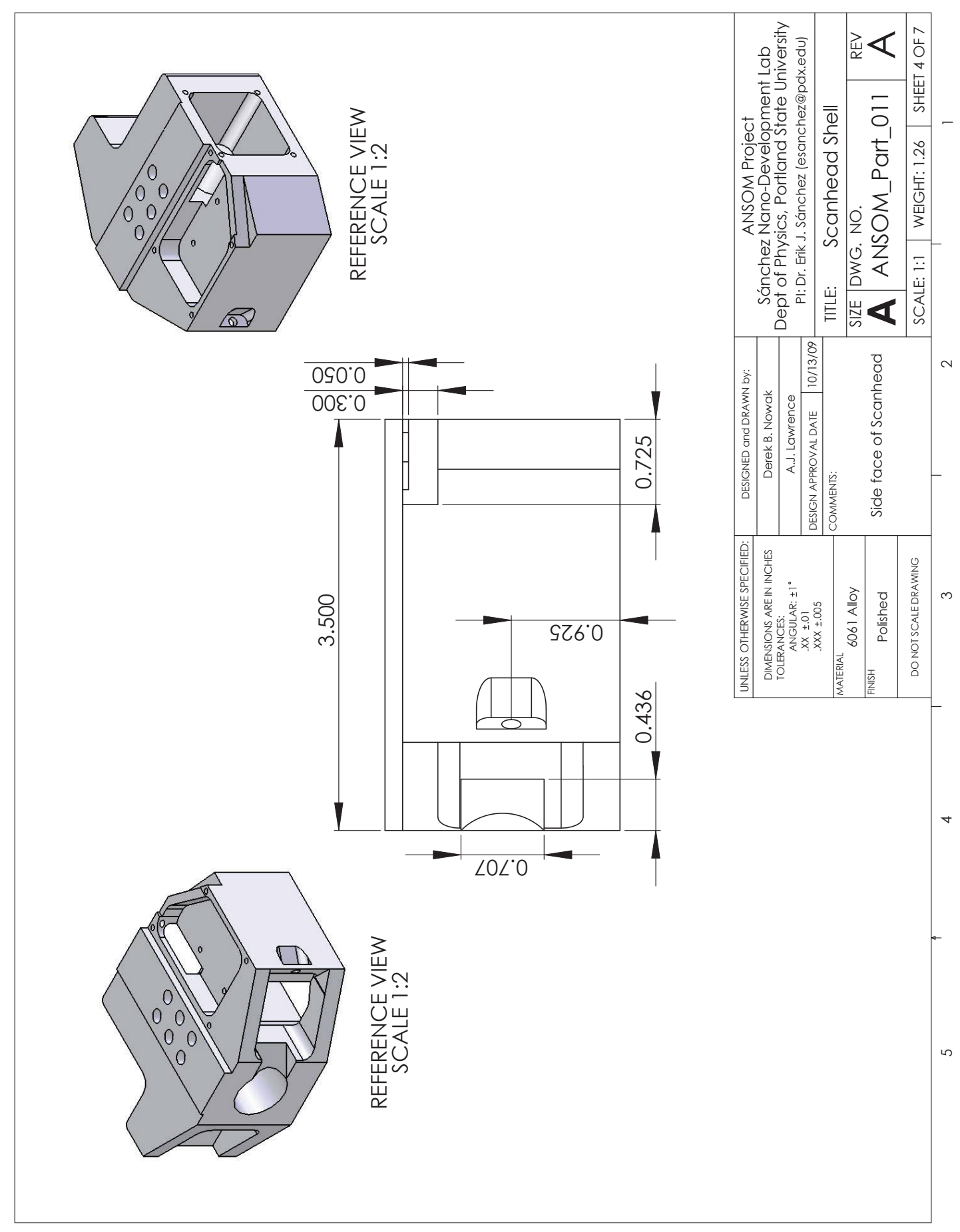




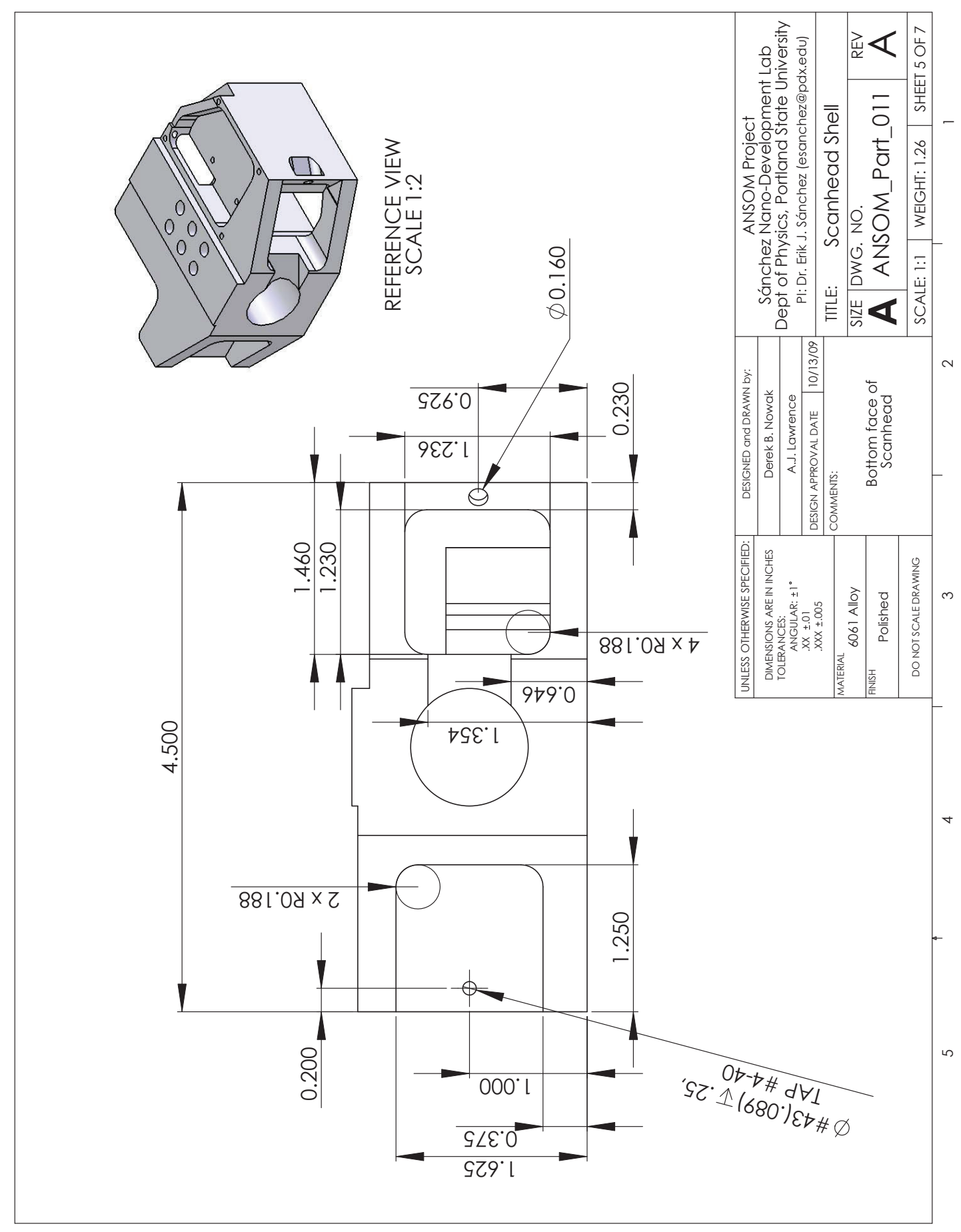




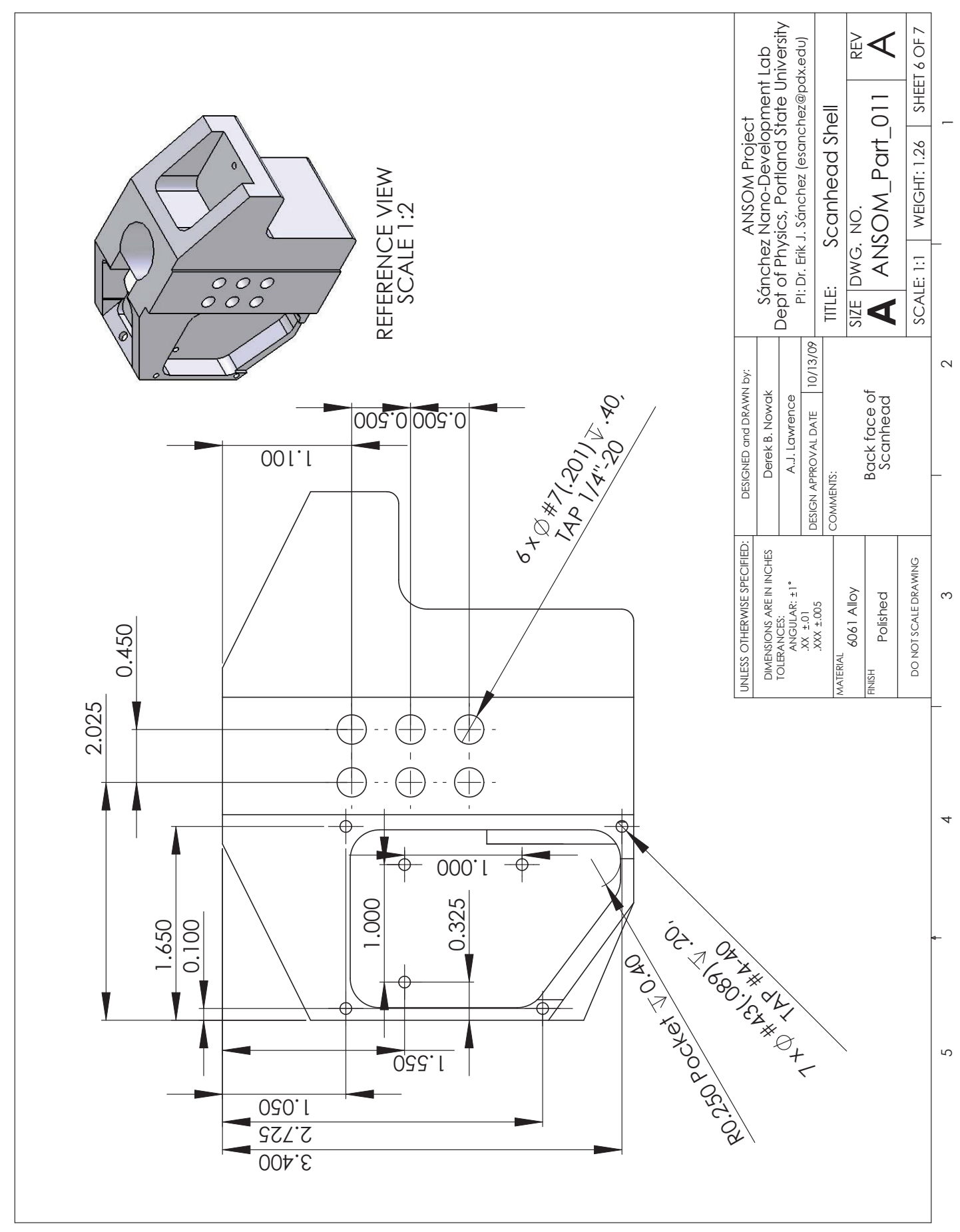




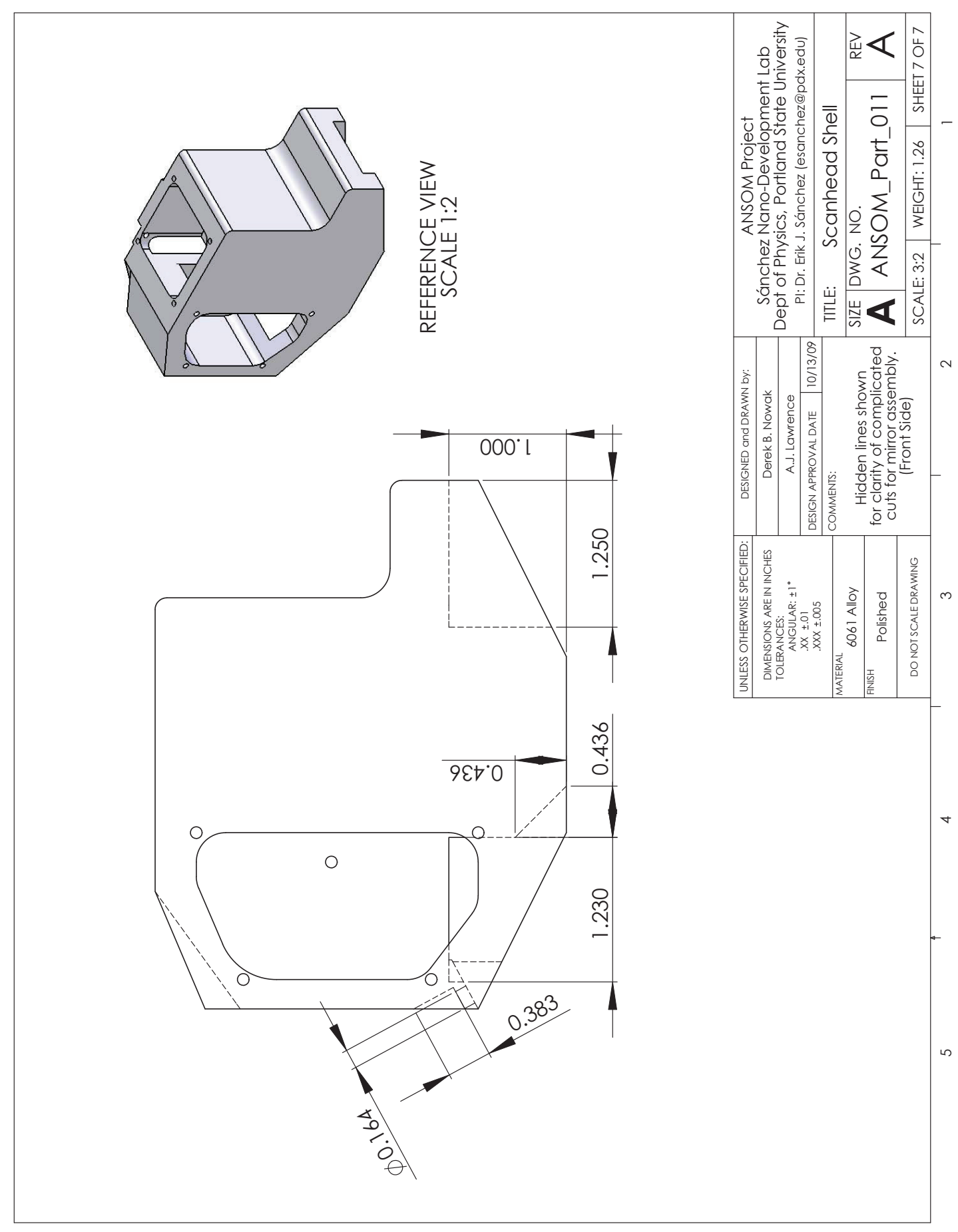




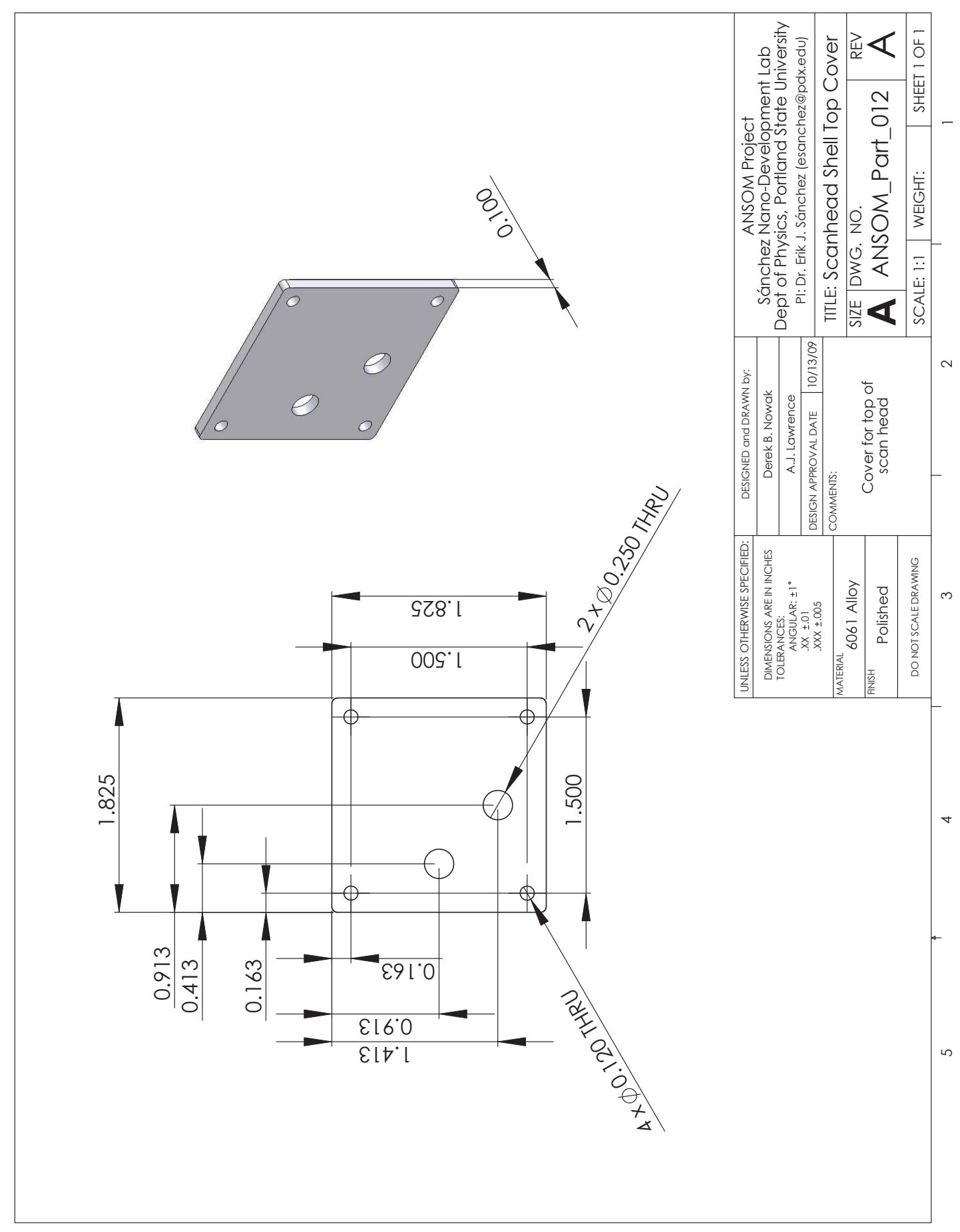




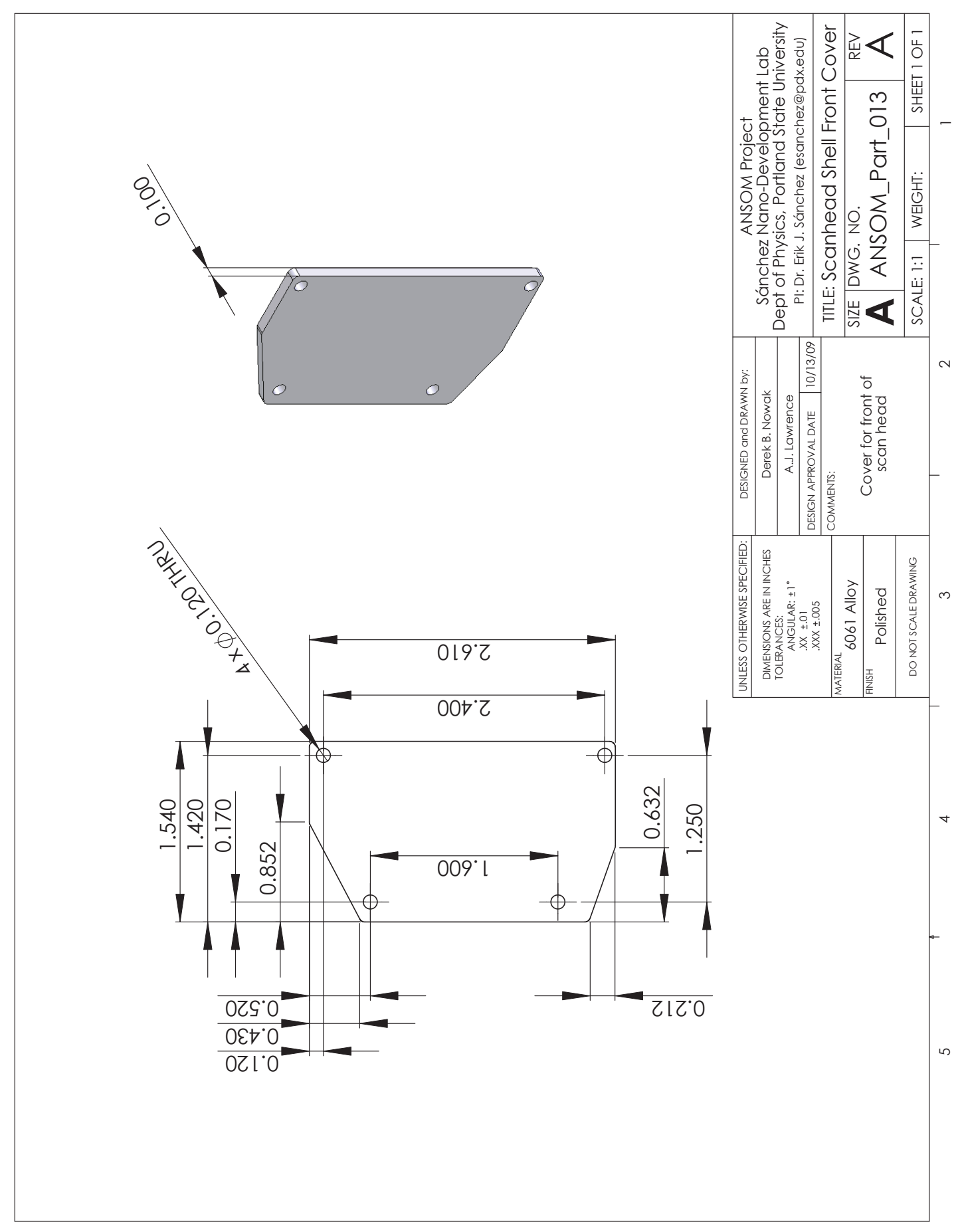




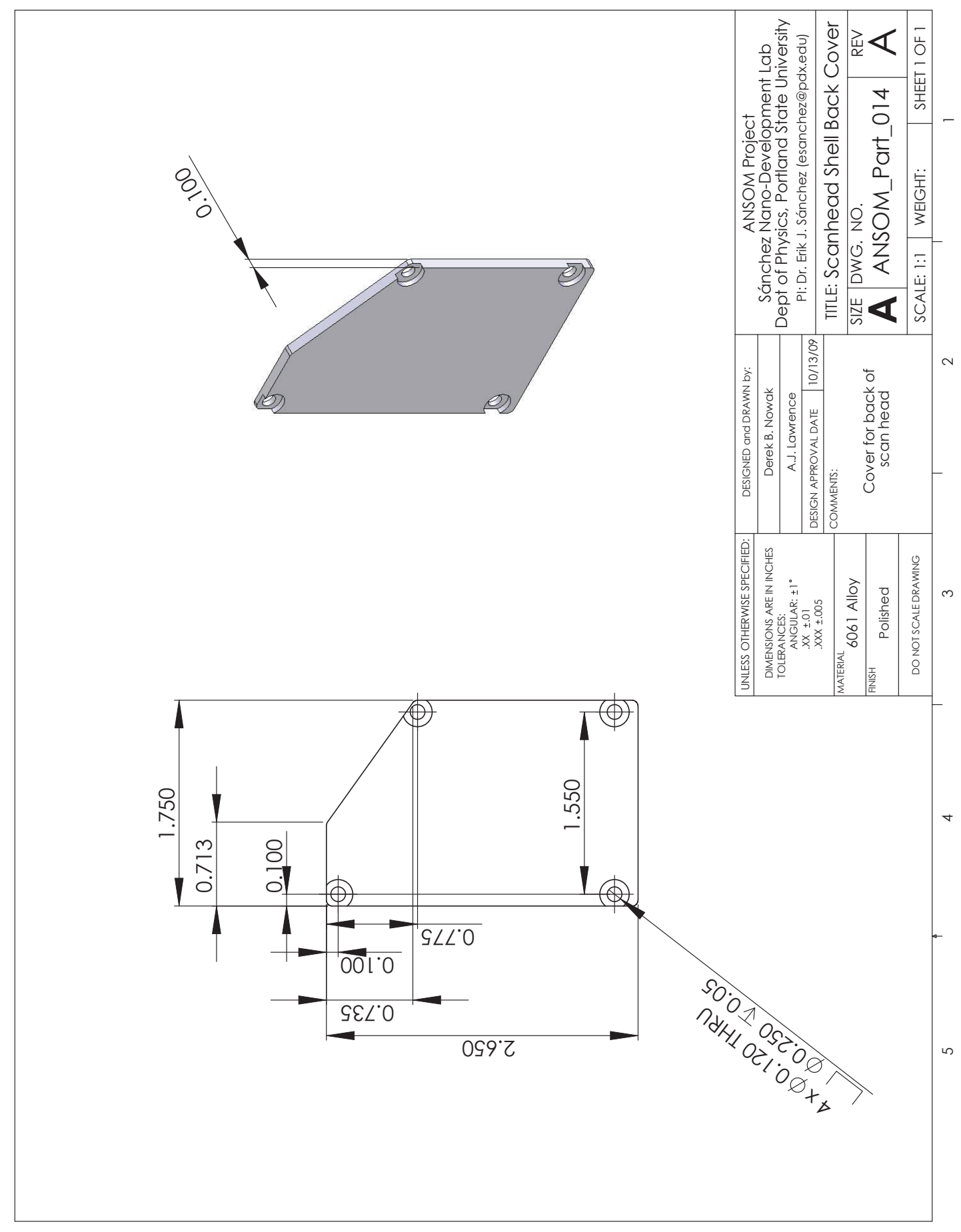




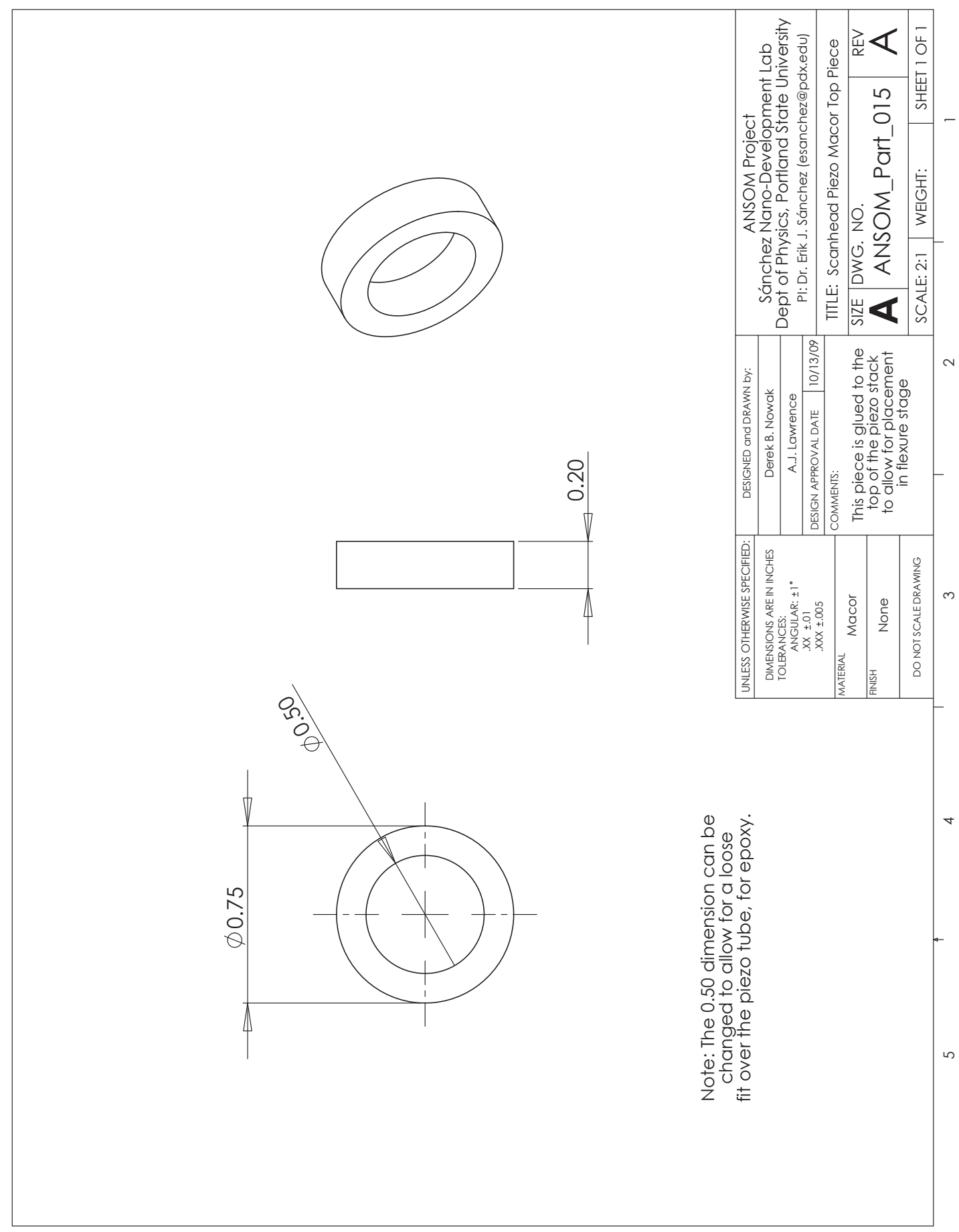




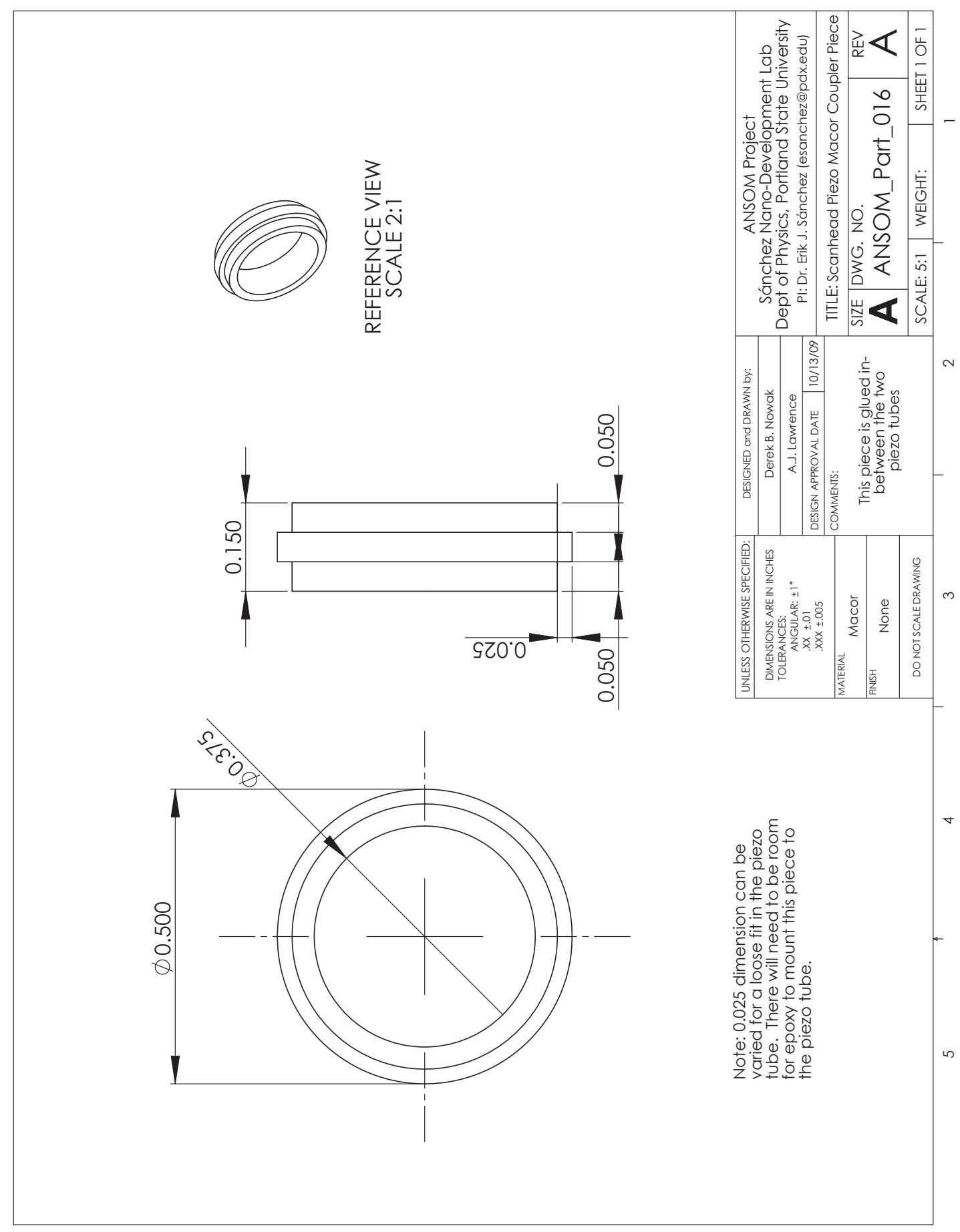




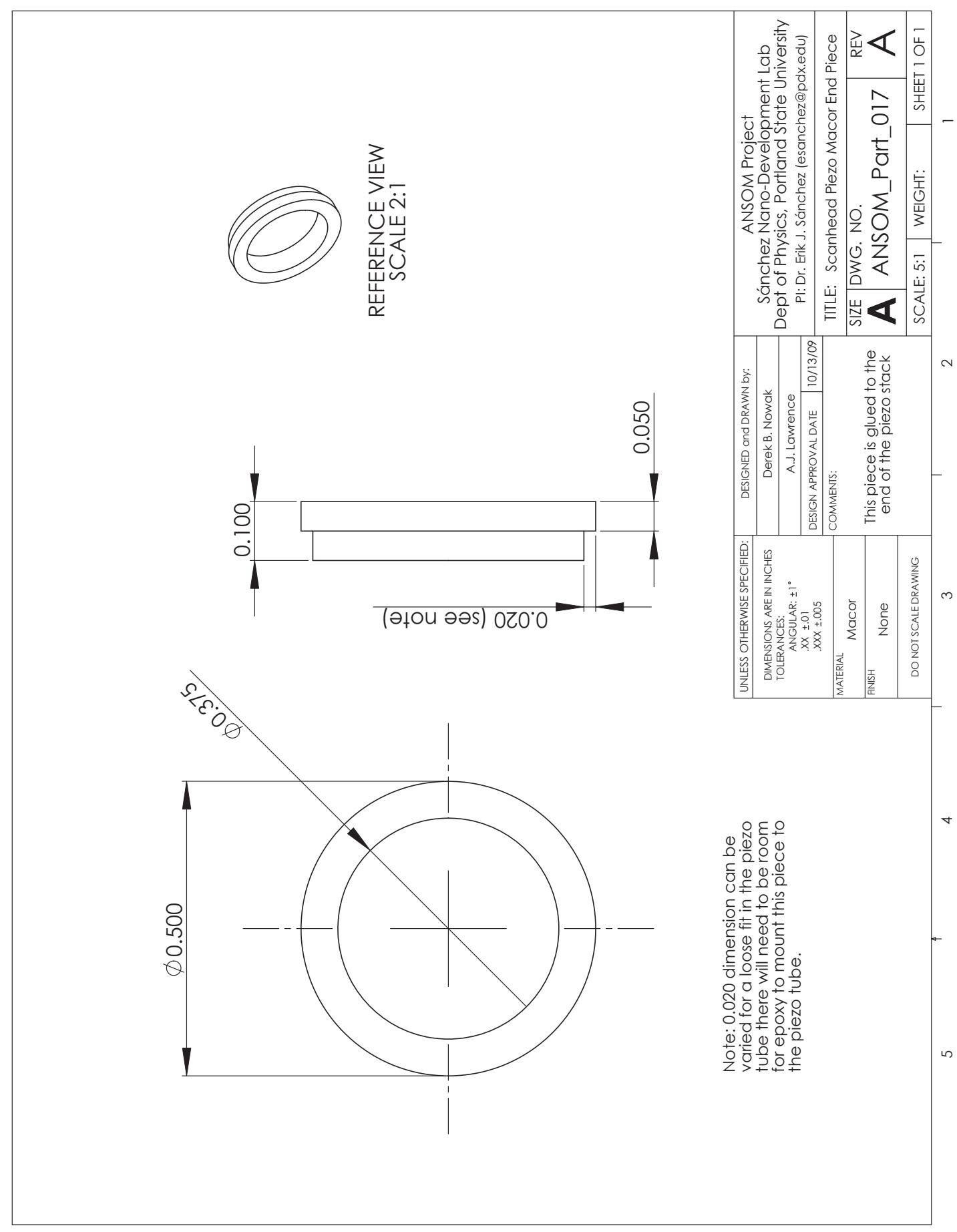




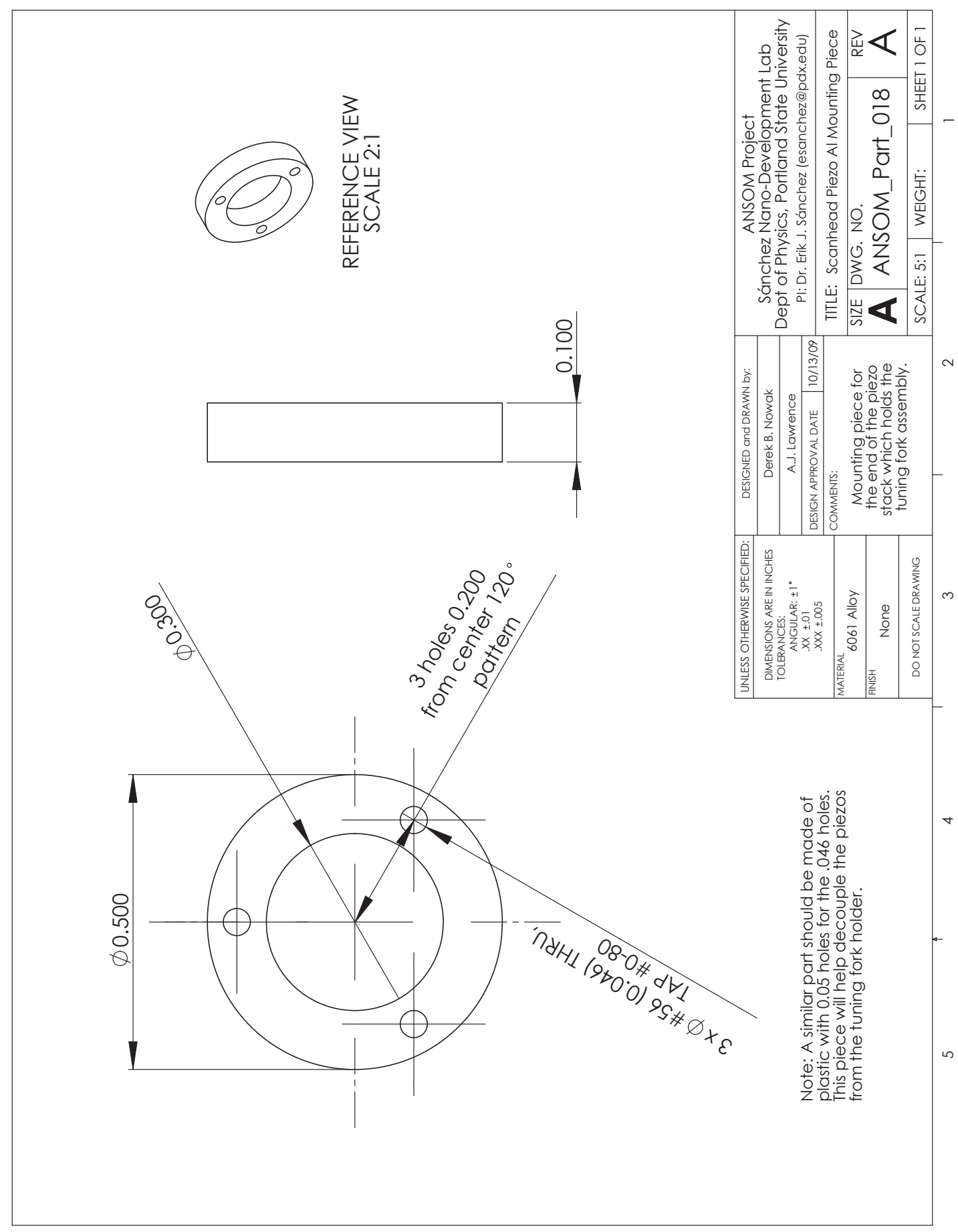




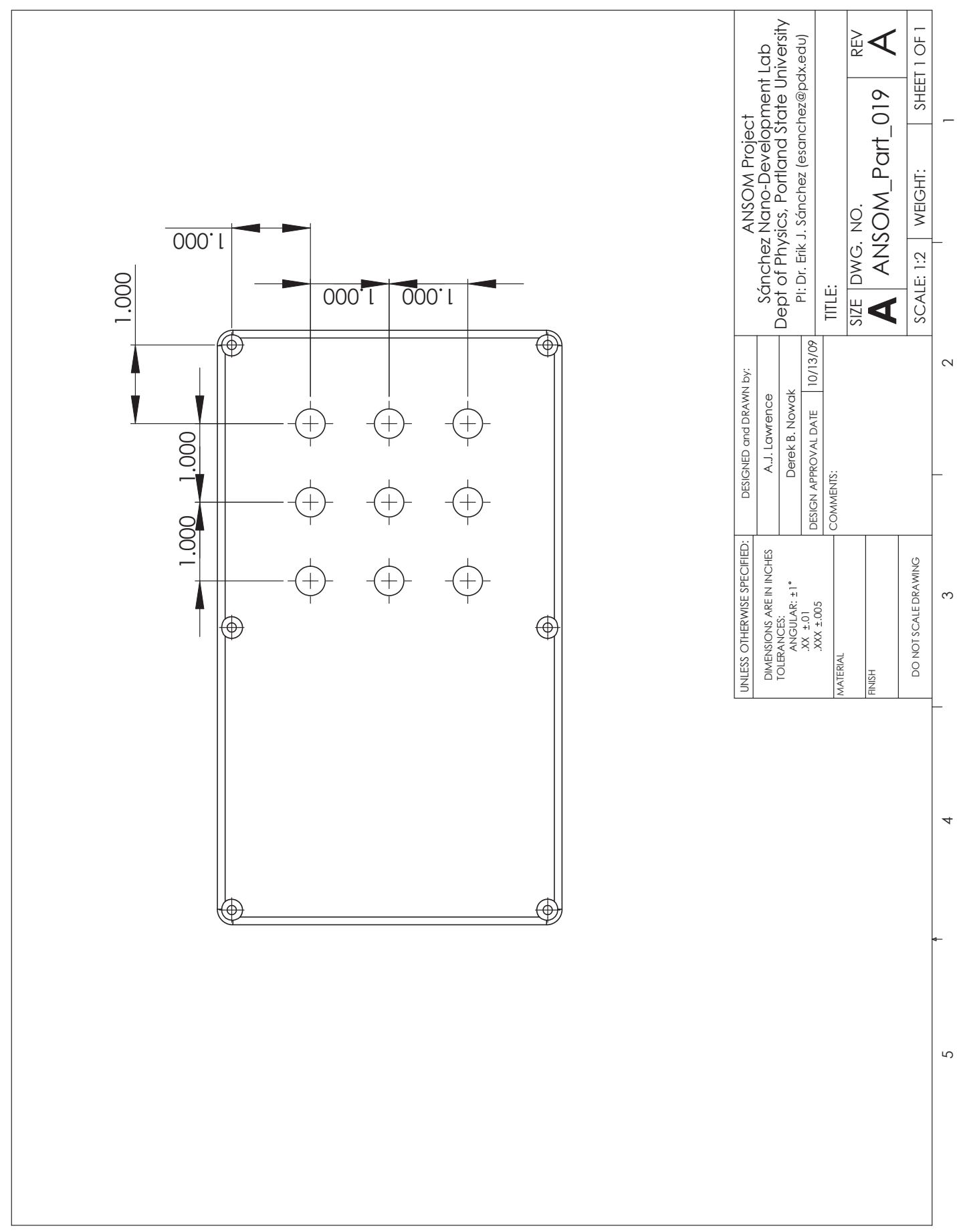




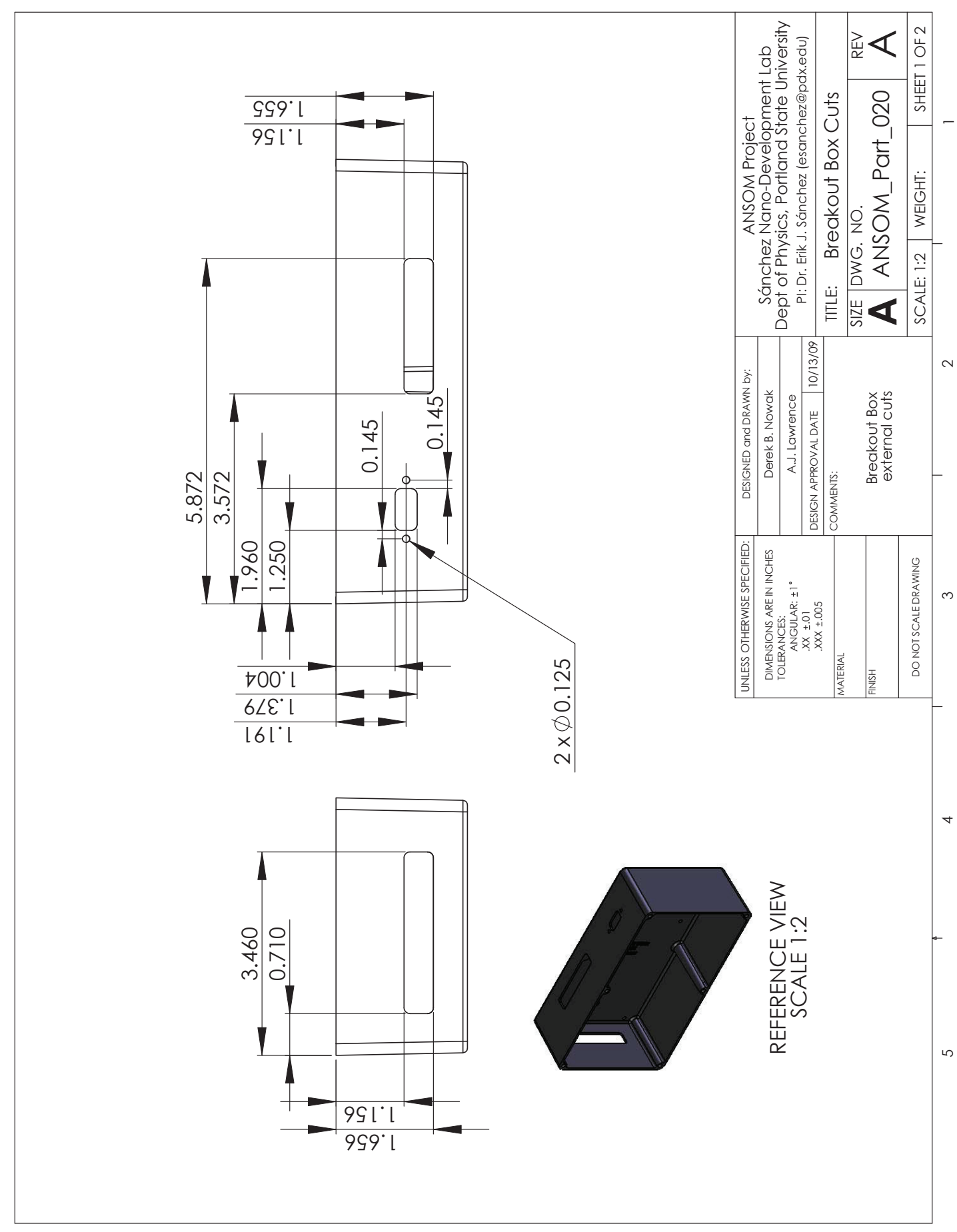




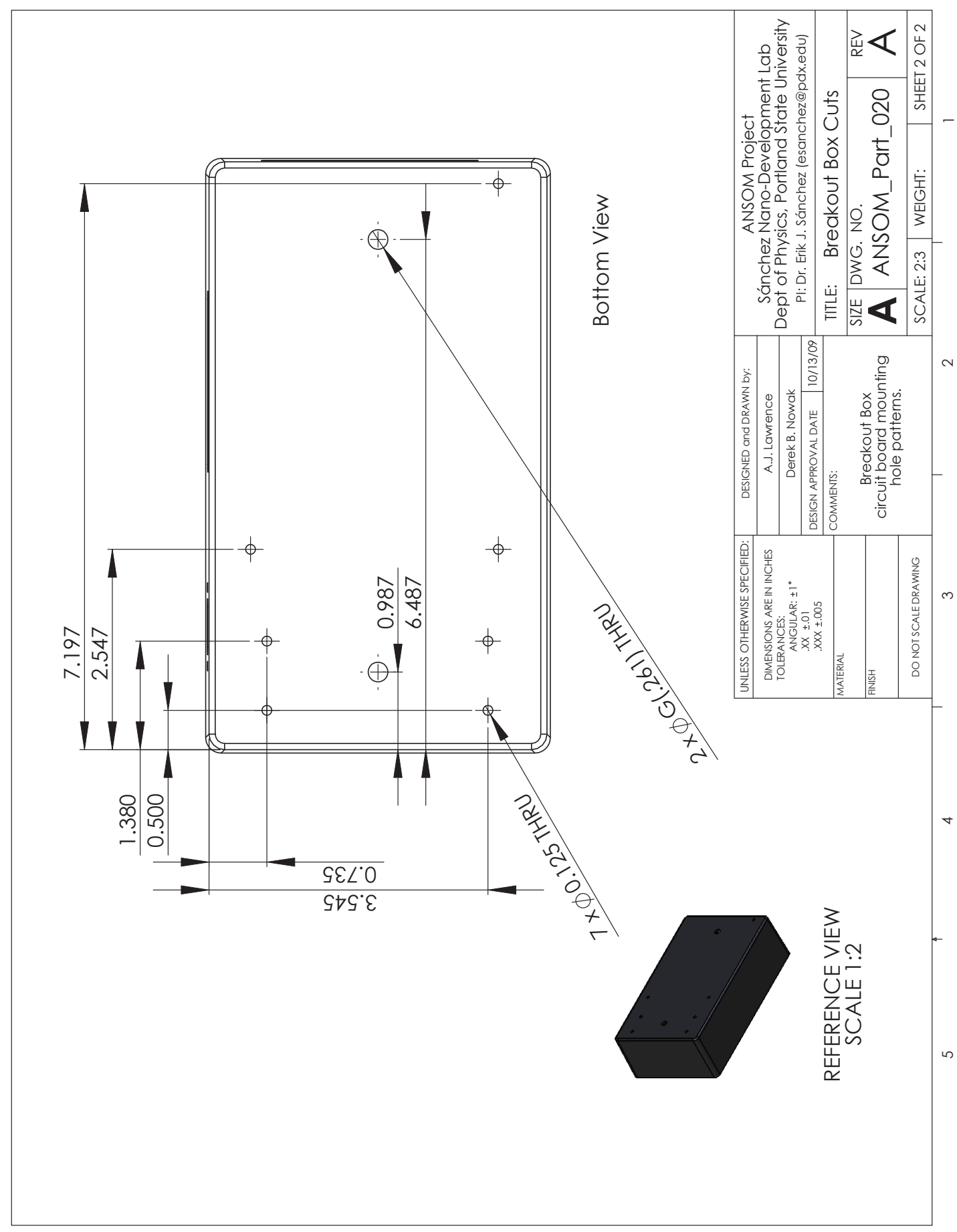




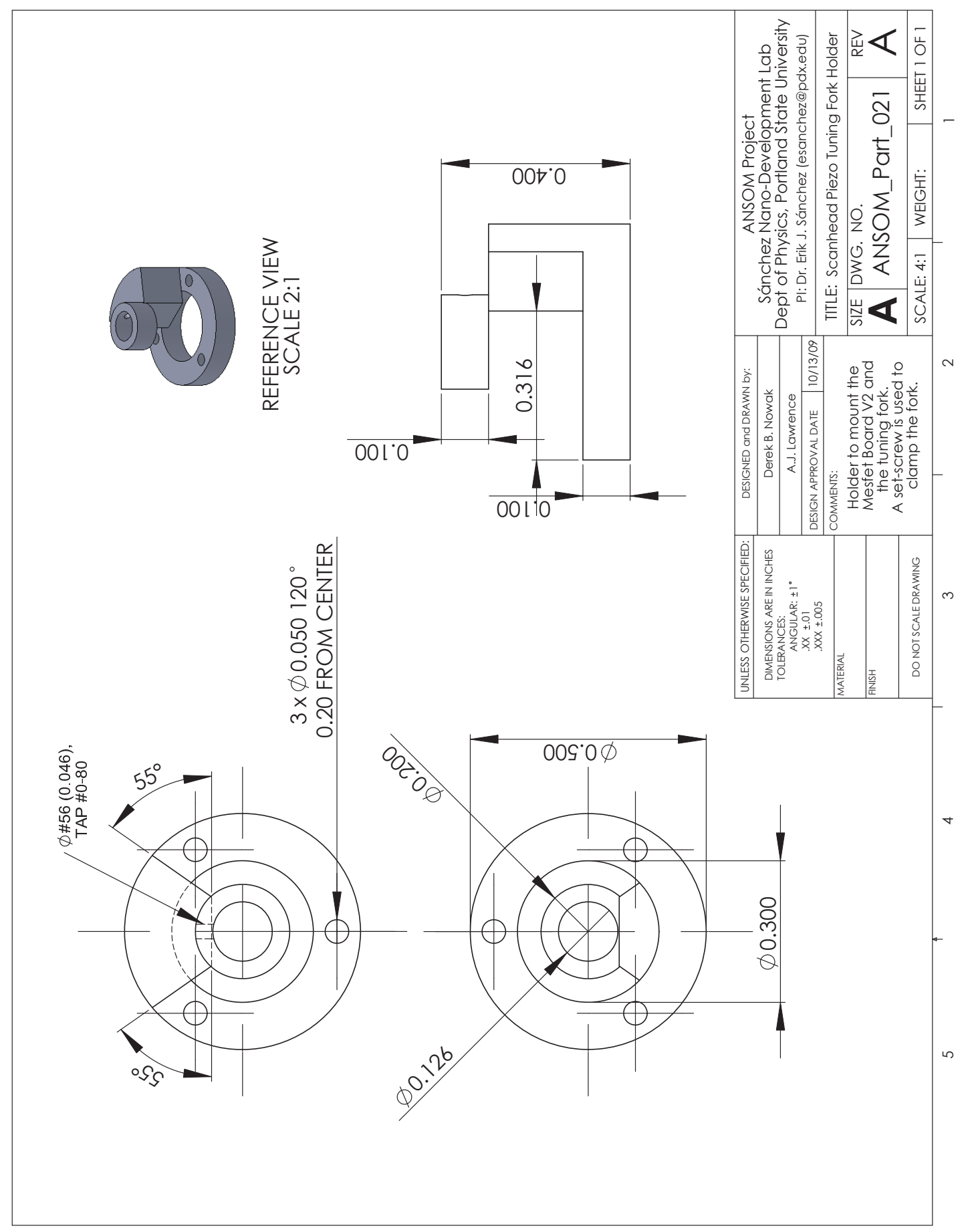




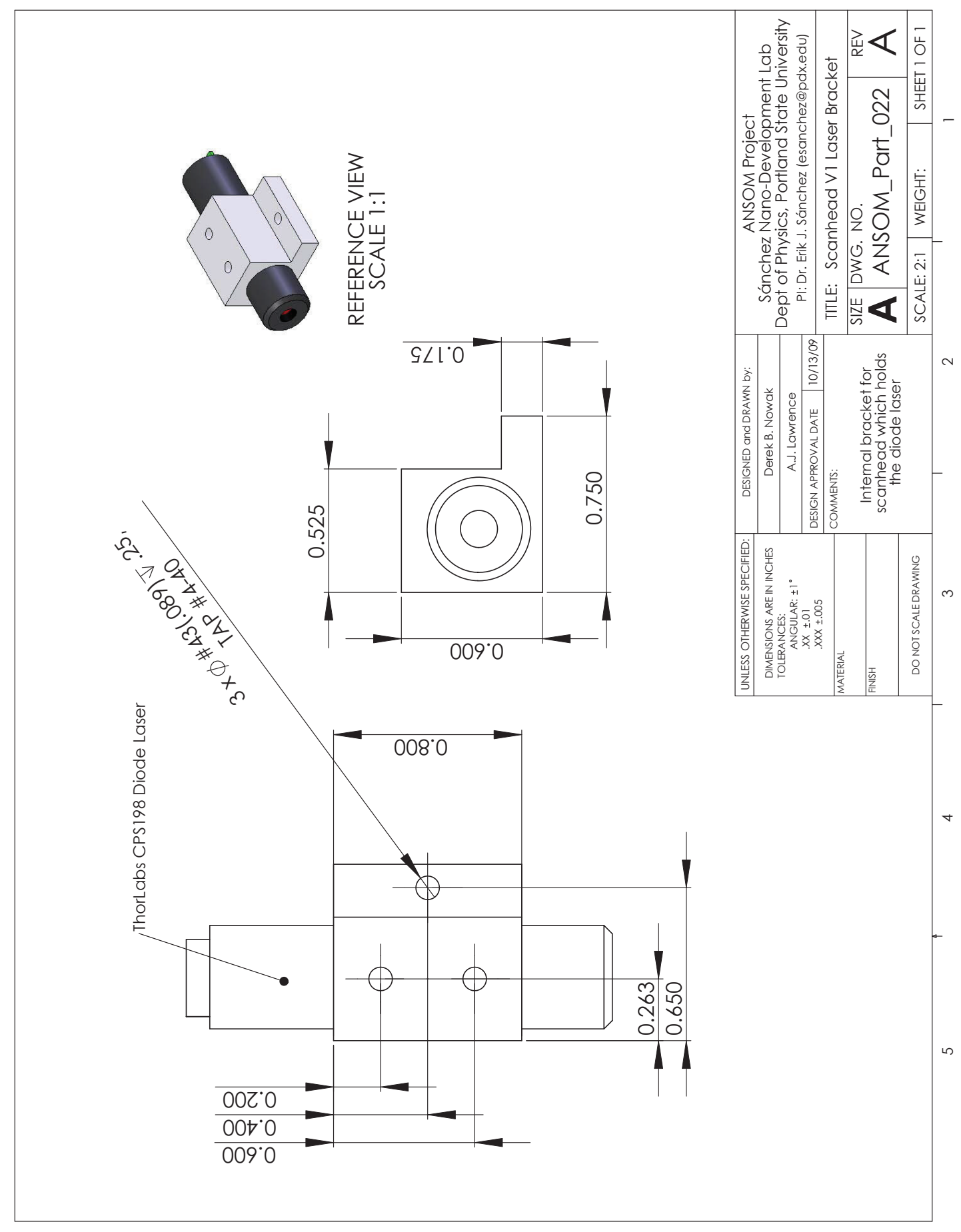




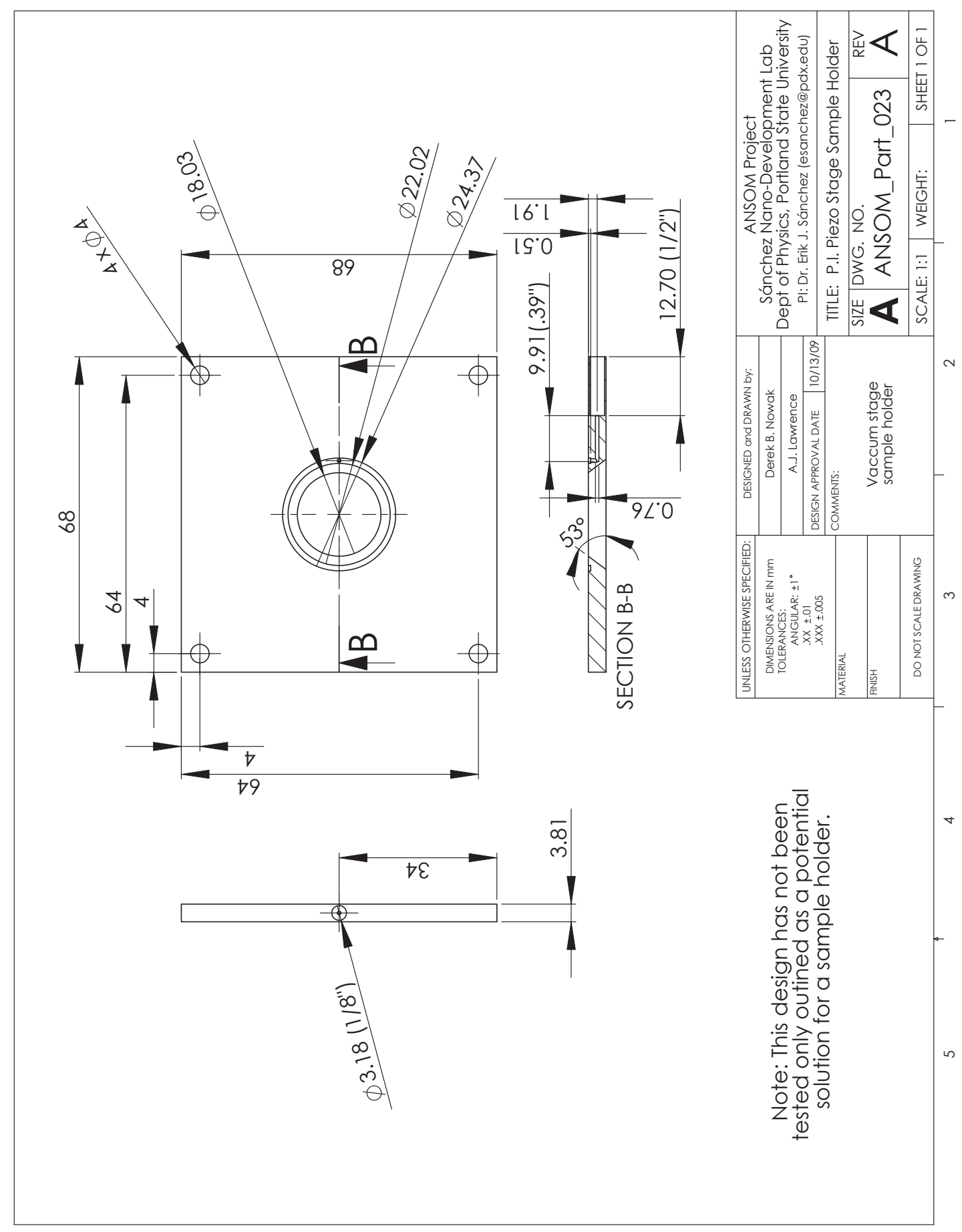

